\title{
Space charge modeling of dense electron beams with large energy spreads
}

\author{
G. Fubiani, ${ }^{*}$ J. Qiang, E. Esarey, ${ }^{\dagger}$ and W. P. Leemans ${ }^{\dagger}$ \\ Ernest Orlando Lawrence Berkeley National Laboratory, University of California, Berkeley, California 94720, USA
}

G. Dugan

Cornell University, Ithaca, New York 14853, USA

(Received 30 January 2006; published 22 June 2006)

\begin{abstract}
Theoretical and numerical studies of the transport in vacuum of multi-nC, multi-MeV electron beams are performed using several methods, including envelope models, a novel semianalytic approach using ellipsoidal shell decomposition, a modified electrostatic particle-in-cell method, and a point-to-point interaction model. The effects of space-charge forces on the longitudinal and transverse bunch properties are evaluated for various bunch lengths, energies, energy spreads, and charges. An evaluation of the various methods for studying space-charge effects in large energy spread, high charge beams is summarized. Examples are given for beam distributions typical of those generated by plasma-based accelerators. It is found that, for the highly correlated beams produced in the self-modulated regime, the high energy portion of the beam can gain significant energy while propagating in vacuum due to spacecharge effects.
\end{abstract}

DOI: 10.1103/PhysRevSTAB.9.064402

PACS numbers: 52.38.Kd

\section{INTRODUCTION}

The propagation in vacuum of dense electron beams, typical of those produced by plasma-based accelerators, is examined. Such beams are typically very compact, with a relatively high charge per bunch and a significant energy spread (depending on experimental scheme). An important issue is to understand how these beams evolve as they exit the plasma. This includes the evolution of the bunch shape, duration, energy spread, and emittance, as well as the time scale for this evolution. This paper concentrates on two types of beams relevant to laser wakefield accelerators (LWFAs).

The first type is typical of that produced by the selfmodulated LWFA (SM-LWFA) [1]. The SM-LWFA typically uses a single laser pulse that is long compared to the plasma wavelength, $L>\lambda_{p}$, and relies on self-trapping to inject electrons into the plasma wakefield. Here $L$ is the laser pulse length and $\lambda_{p}=2 \pi / k_{p}=2 \pi c / \omega_{p}$, where $\omega_{p}=\left(n_{0} e^{2} / \epsilon_{0} m_{e}\right)^{1 / 2}$ is the plasma frequency, $n_{0}$ is the ambient plasma density, $m_{e}$ the electron rest mass, $e$ the electron charge, $c$ the speed of light in vacuum, and $\epsilon_{0}$ is the permittivity of free space. Because the electrons are self-injected, the resulting bunch typically has a large energy spread, characterized by an exponential distribution in energy with a temperature ranging from 1-10's of $\mathrm{MeV}$. The bunch can have high charge $\sim 1-10 \mathrm{nC}$, with typical transverse dimensions $\sim 10 \mu \mathrm{m}$, and bunch half-length $\sim 10 \mu \mathrm{m}$, giving a high number density $\sim 2.5 \times$ $10^{19} \mathrm{~cm}^{-3}$.

\footnotetext{
*Also at University of Paris XI, 91405 Orsay Cedex, France.

${ }^{\dagger}$ Also at University of Nevada, Reno, Nevada 89557, USA.
}

The second type of beam is typical of that predicted to be produced by LWFAs that utilize optical injection, such as in the colliding pulse injector (CPI) [2-5]. The CPI scheme is predicted to produce bunches of small relative energy spread by using two to three short laser pulses of length $L \lesssim \lambda_{p}$. The drive pulse generates the wake, and the beating of the backward pulse with the drive pulse (or a third pulse) injects electrons with a small phase spread into the wakefield. This scheme offers detailed control of the injection process, and since $L \lesssim \lambda_{p}$, Raman and self-modulation instabilities will be suppressed. The resulting electron bunches carry a charge $\sim 10-200 \mathrm{pC}$, with an average energy $>10 \mathrm{MeV}$, and a relative energy spread of $1 \%-10 \%$. The bunch typical transverse dimensions are $\sim 10 \mu \mathrm{m}$, and the bunch half-length is $\sim 2.5 \mu \mathrm{m}$, corresponding to a number density of $\sim 2.5 \times 10^{17} \mathrm{~cm}^{-3}$. This is 2 orders of magnitude smaller than in the selfmodulated regime, but the longitudinal quality of the beam is much better because of the small relative energy spread.

Several algorithms for computing space-charge forces have been proposed and intensively used over the past years to model electron beam dynamics in linacs and storage rings [6-8]. Such methods generally assume that the electron beam has a small energy spread. In this case there is a single coordinate system (frame) in which all beam particles are nonrelativistic, simplifying considerably the calculation of electromagnetic self-fields. This method may not be directly applied to beams with large energy spreads and it is consequently useful to develop new models allowing for fast computation of beam dynamics without the necessary use of fully explicit techniques such as particle-in-cell (PIC) codes $[9,10]$ that are known to be computationally costly. 
In this paper, the evolution of the electron bunch in vacuum and in the absence of external forces is considered. The assumptions and numerical methods (envelope equations, ellipsoidal shell decomposition, electrostatic PIC method, and point-to-point interactions) are summarized from Sec. II to Sec. V. Specifically, Sec. II examines a rapid and innovative method for the calculation of space-charge effects, assuming a paraxial approximation but capable of handling beams with a large energy distribution [11]. This method makes assumptions about the position space ellipsoidal symmetry properties of the charge distribution and is valid for a large parameter regime. In order to obtain simple analytical expressions for the space-charge forces, this section is further specialized to charge distributions with radial symmetry about the axis of propagation. Section III gives a summary of the envelope model followed by the descriptions, in Sec. IV, of a modified electrostatic particle-in-cell code. The latter is an extension of the model discussed in Sec. II and does not make any assumptions on the spatial symmetries of the electron beam. In Sec. V, a three-dimensional (3D) point-to-point interaction (PPI) approach will be discussed. This method applies to large energy spread beams and does not require the paraxial approximation. Section VI examines some electron sources produced by CPI and SM-LWFA configurations, Sec. VII compares PPI and PIC codes for the simulation of space-charge dominated beams, and Sec. VIII discusses (i) both limitations and possible generalizations of the PPI model and (ii) emittance growth for beams with large energy spreads. Appendices are also included to discuss further aspects of these topics.

\section{BASIC EQUATIONS FOR THE SHELL MODEL}

In this section, a novel technique is described that uses an ellipsoidal model for the beam charge distribution and allows the treatment of arbitrarily large energy spreads.

\section{A. Structure of the code}

The six-dimensional (6D) beam phase-space distribution function is represented numerically as a collection of macroparticles [11]. The phase-space coordinates of these macroparticles evolve under the influence of the collective space-charge forces. To compute these space-charge forces, the range of longitudinal momenta spanned by the beam is divided into a series of bins. Each bin has a normalized longitudinal momentum width $\Delta u_{z}=$ $\Delta p_{z} /(m c) \ll 1$. Consequently, in the rest frame of each bin, the macroparticles within that bin are nonrelativistic, and the space-charge forces in that frame may be computed from the electrostatic field of the macroparticle charge distribution.

The macroparticle charge distribution is modeled within a bin as a series of concentric ellipsoidal shells. The parameters of these ellipsoidal shells, such as the rootmean-square (RMS) radii, the density, and the average position, are obtained by statistical averages over the selected macroparticles. Analytical expressions are used for the electrostatic field for each ellipsoidal shell [12], which enables a fast computational algorithm. The total electrostatic field due to the macroparticles is given by the sum over the ellipsoidal shells.

To calculate the force on a given macroparticle at each time step, we iterate through each momentum bin, and, from the electrostatic fields of the macroparticles in that bin, calculate the space-charge force on the given macroparticle in the rest frame of that bin. We then transform this space-charge force into the lab frame. The total force on the macroparticle is the vector sum of the Lorentztransformed space-charge forces due to all the momentum bins. This procedure is repeated for each macroparticle, giving all the forces needed to evolve the macroparticle distribution to the next time step.

Note that the binning by momentum, and the calculation of the ellipsoid parameters characterizing the macroparticle charge distribution associated with each bin, is done at every time step.

\section{B. Adaptive longitudinal momentum grid}

In order to be able to approximate the space-charge fields as purely electrostatic, the collection of charges generating these fields must be nonrelativistic in their common rest frame. Such an approximation requires that the normalized momentum spread in the rest frame be small, namely $\Delta p_{\mathrm{cm}} /(m c)=\Delta u_{\mathrm{cm}}=\eta \ll 1$. This condition is achieved in the code by using an adaptive binning technique to break the longitudinal momentum distribution up into bins (the transverse momentum spread is small because of the small angular spread in the beam). This can be done as follows: Performing a Lorentz transformation on the 4-vector normalized momentum $u^{\alpha} \equiv(\gamma, \gamma \beta)$ gives the relationship between the momentum spread in the laboratory frame $\Delta u_{z}$ and the momentum spread in the rest frame $\Delta u_{z}^{\mathrm{cm}}$ [7],

$$
\Delta u_{z}^{\mathrm{cm}} \simeq \eta=\gamma\left(\Delta u_{z}-\beta \Delta \gamma\right) .
$$

Assuming the paraxial approximation $\left(\left|\beta_{\perp}\right| \ll\left|\beta_{z}\right|\right)$ gives $\gamma^{2} \simeq 1+u_{z}^{2}$ and $\gamma \Delta \gamma \simeq u_{z} \Delta u_{z}$ or $\Delta \gamma \simeq \beta \Delta u_{z}$. Inserting $\Delta \gamma \simeq \beta \Delta u_{z}$ into Eq. (1) yields

$$
\Delta u_{z} \simeq \eta \gamma \simeq \eta \sqrt{1+u_{z}^{2}} .
$$

Using Eq. (2), the bin width requirement in terms of laboratory longitudinal $(z)$ momentum, for a laboratory momentum $u_{z_{k}}$ in bin $k$, is found to be

$$
\Delta u_{z}=u_{z_{k+1}}-u_{z_{k}} \simeq \eta \sqrt{1+u_{z_{k}}^{2}}
$$

and in the limiting case $\eta \ll 1$,

$$
\frac{d u_{z}(k)}{d k} \simeq \eta \sqrt{1+u_{z}^{2}}
$$


hence, the longitudinal momentum bins are defined by

$$
u_{z_{k}}=\sinh \left(k \eta+\sinh ^{-1} u_{z_{0}}\right) .
$$

The bin index for a given momentum $u_{z}$ is

$$
k=\left[\frac{\sinh ^{-1} u_{z}-\sinh ^{-1} u_{z_{0}}}{\eta}\right\rfloor .
$$

Note that $u_{z_{0}}$ is taken as the lowest momentum of the distribution function; it defines the first bin.

\section{Total force calculations}

For the macroparticles in momentum bin $k$, we compute in the laboratory frame the transverse ( $a_{1 k}$ and $a_{2 k}$ along $x$ and $y$ ) and longitudinal ( $a_{3 k}$ along the coordinate $z$ ) RMS sizes of the bunch, and the mean longitudinal position of the bunch $\left(\bar{z}_{k}\right)$. As noted above, radial symmetry is assumed, so that $a_{1 k}=a_{2 k} \equiv a_{k}$, in order to be able to use a simple analytical solution for the fields.

The quantities $a_{k}$ and $a_{3 k}$ define an ellipsoid that models the shape of the macroparticle distribution. This ellipsoid is decomposed into a number of concentric ellipsoidal shells $s_{k}=1,2, \ldots, N_{k}$. The inner boundary of shell $s_{k}$ is designated $m_{0}\left(s_{k}\right) a_{k}$ radially and $m_{0}\left(s_{k}\right) a_{3 k}$ longitudinally; and the outer boundary is $m_{1}\left(s_{k}\right) a_{k}$ radially and $m_{1}\left(s_{k}\right) a_{3 k}$ longitudinally. The numbers $m_{0}\left(s_{k}\right)$ and $m_{1}\left(s_{k}\right)$, that range from 0 to $\mu_{m}$ [maximum ellipsoidal coordinate of the distribution function $\mu^{2}=\left(x^{2}+y^{2}\right) / a_{k}^{2}+(z-$ $\left.\bar{z}_{k}\right)^{2} / a_{3 k}^{2}$, are chosen such that the volume of each of the ellipsoidal shells is the same. The density of macroparticles $\rho_{0_{s_{k}}}$ within shell $s_{k}$ is calculated numerically from the macroparticle distribution. Using the Lorentz transformation

$$
\left\{\begin{array}{l}
\mathbf{E}_{s_{k}}=\gamma_{k} \mathbf{E}_{s_{k}}^{\prime}-\gamma_{k}^{2}\left(\gamma_{k}+1\right)^{-1} \beta_{k}\left(\beta_{k} \cdot \mathbf{E}_{s_{k}}^{\prime}\right), \\
c \mathbf{B}_{s_{k}}=\gamma_{k} \beta_{k} \times \mathbf{E}^{\prime},
\end{array}\right.
$$

one can calculate the electromagnetic field produced by an ellipsoidal shell, acting at the coordinate $\{x, y, z\}$ in the laboratory frame,

$$
\begin{gathered}
E_{s_{k}}^{(x)}=\frac{\rho_{0_{s_{k}}}}{2 \epsilon_{0}} a_{k}^{2} \gamma_{k} a_{3_{k}} x A_{s_{k}}\left(u_{1_{k}}, u_{0_{k}}\right), \\
E_{s_{k}}^{(y)}=\frac{\rho_{0_{s_{k}}}}{2 \epsilon_{0}} a_{k}^{2} \gamma_{k} a_{3_{k}} y A_{s_{k}}\left(u_{1_{k}}, u_{0_{k}}\right), \\
E_{s_{k}}^{(z)}=\frac{\rho_{0_{s_{k}}}}{2 \epsilon_{0}} a_{k}^{2} \gamma_{k} a_{3_{k}}\left(z-\bar{z}_{k}\right) A_{{s_{s}}_{k}}\left(u_{1_{k}}, u_{0_{k}}\right),
\end{gathered}
$$

and

$$
\begin{gathered}
c B_{s_{k}}^{(x)}(x, y, z)=-\beta_{k} E_{s_{k}}^{(y)}(x, y, z), \\
c B_{s_{k}}^{(y)}(x, y, z)=\beta_{k} E_{s_{k}}^{(x)}(x, y, z),
\end{gathered}
$$

$$
c B_{s_{k}}^{(z)}(x, y, z)=0,
$$

in which $A_{s_{k}}\left(A_{3_{s_{k}}}\right)$ is the transverse (longitudinal) spacecharge coefficient (see Appendices A and B for further details), and $\gamma_{k}=\left(1-\beta_{k}^{2}\right)^{-1 / 2}$ is the relativistic factor of the $k$ th bin. The space-charge coefficients are $A_{s_{k}}\left(u_{1_{k}}, u_{0_{k}}\right)=g_{0_{k}}\left(u_{0_{k}}\right)-g_{0_{k}}\left(u_{1_{k}}\right), \quad A_{3_{s_{k}}}\left(u_{1_{k}}, u_{0_{k}}\right)=$ $h_{0_{k}}\left(u_{0_{k}}\right)-h_{0_{k}}\left(u_{1_{k}}\right)$, with

$$
\begin{aligned}
g_{0_{k}}(u)= & \left(a_{k}^{2}-\gamma_{k}^{2} a_{3_{k}}^{2}\right)^{-1}\left\{\frac{\sqrt{\gamma_{k}^{2} a_{3_{k}}^{2}+u}}{a_{k}^{2}+u}\right. \\
& \left.+\frac{\tan ^{-1}\left[\sqrt{\gamma_{k}^{2} a_{3_{k}}^{2}+u} / \sqrt{a_{k}^{2}-\gamma_{k}^{2} a_{3_{k}}^{2}}\right]}{\sqrt{a_{k}^{2}-\gamma_{k}^{2} a_{3_{k}}^{2}}}\right\},
\end{aligned}
$$

and

$$
\begin{aligned}
h_{0_{k}}(u)= & \frac{2}{a_{k}^{2}-\gamma_{k}^{2} a_{3_{k}}^{2}}\left\{\left(\gamma_{k}^{2} a_{3_{k}}^{2}+u\right)^{-1 / 2}\right. \\
& \left.+\frac{\tan ^{-1}\left[\sqrt{\gamma_{k}^{2} a_{3_{k}}^{2}+u} / \sqrt{a_{k}^{2}-\gamma_{k}^{2} a_{3_{k}}^{2}}\right]}{\sqrt{a_{k}^{2}-\gamma_{k}^{2} a_{3_{k}}^{2}}}\right\} .
\end{aligned}
$$

We also have $u_{0_{k}}(x, y, z)=\lambda_{k}\left(x, y, z, m_{0}^{2}\left(s_{k}\right)\right) / m_{0}^{2}\left(s_{k}\right)$, and $u_{1_{k}}(x, y, z)=\lambda_{k}\left(x, y, z, m_{1}^{2}\left(s_{k}\right)\right) / m_{1}^{2}\left(s_{k}\right)$, where

$$
\begin{aligned}
2 \lambda_{k}\left(x, y, z, m^{2}\right)= & x^{2}+y^{2}+\gamma_{k}^{2}\left(z-\bar{z}_{k}\right)^{2}-m^{2} a_{k}^{2} \\
& -m^{2} \gamma_{k}^{2} a_{3 k}^{2}+\left[\left(x^{2}+y^{2}+\gamma_{k}^{2}\left(z-\bar{z}_{k}\right)^{2}\right.\right. \\
& \left.-m^{2} a_{k}^{2}-m^{2} \gamma_{k}^{2} a_{3 k}^{2}\right)^{2}+4 m^{2} \gamma_{k}^{2}\left(a _ { 3 k } ^ { 2 } \left(x^{2}\right.\right. \\
& \left.\left.\left.+y^{2}\right)+a_{k}^{2}\left(\left(z-\bar{z}_{k}\right)^{2}-m^{2} a_{3 k}^{2}\right)\right)\right]^{1 / 2} .
\end{aligned}
$$

If $\quad\left(x^{2}+y^{2}\right) / a_{k}^{2}+\left(z-\bar{z}_{k}\right)^{2} / a_{3 k}^{2}<m^{2}$,

then $\lambda_{k}\left(x, y, z, m^{2}\right)=0$.

Knowing the electromagnetic field from a single shell, one can easily deduce the resulting total force per unit charge acting on a given macroparticle by summing over all ellipsoidal shells $s_{k}$, and then over all momentum bins $k$ :

$$
\begin{gathered}
F_{x}=\sum_{k}\left(1-\beta_{z} \beta_{k}\right) \sum_{s_{k}} E_{s_{k}}^{(x)}, \\
F_{y}=\sum_{k}\left(1-\beta_{z} \beta_{k}\right) \sum_{s_{k}} E_{s_{k}}^{(y)}, \\
F_{z}=\sum_{k} \sum_{s_{k}} E_{s_{k}}^{(z)}+\beta_{x} \sum_{k} \beta_{k} \sum_{s_{k}} E_{s_{k}}^{(x)}+\beta_{y} \sum_{k} \beta_{k} \sum_{s_{k}} E_{s_{k}}^{(y)},
\end{gathered}
$$

where $\beta=\left(\beta_{x}, \beta_{y}, \beta_{z}\right)$ is the macroparticle normalized velocity. 
The shell approach has been compared to several other methods such as the direct integration of the coupled envelope equations [7,13,14], a modified electrostatic PIC code, and a 3D point-to-point interaction code [15]. Each of this methods has its own set of approximations that we review in Sec. III.

\section{ENVELOPE EQUATIONS}

This section gives a summary of the envelope equations derived for both ellipsoidal symmetric beams and finite cylinders of charge. Limitations of this approach will be further discussed in Sec. VI.

\section{A. Coupled envelope equations in ellipsoidal geometry}

In the following we will assume that the electron bunch has an ellipsoidal symmetry with a circular cross section throughout the interaction. The envelope equations can be defined as (see Appendices $\mathrm{C}$ and $\mathrm{D}$ for further details)

$$
\begin{gathered}
\sigma_{\perp}^{\prime \prime}-\frac{3}{2} \frac{N r_{e}}{\gamma_{0}^{2} \beta_{0}^{2}} \lambda_{3} \sigma_{\perp} A\left(\sigma_{\perp}, \gamma_{0} \sigma_{z}\right)-\frac{\tilde{\epsilon}_{x}^{2}}{\sigma_{\perp}^{3}}=0, \\
\sigma_{z}^{\prime \prime}-\frac{3}{2} \frac{N r_{e}}{\gamma_{0}^{2} \beta_{0}^{2}} \lambda_{3} \sigma_{z} A_{3}\left(\sigma_{\perp}, \gamma_{0} \sigma_{z}\right)-\frac{\tilde{\epsilon}_{z}^{2}}{\sigma_{z}^{3}}=0,
\end{gathered}
$$

where $N$ is the number of electrons in the bunch, $r_{e}=$ $e^{2} /\left(4 \pi \epsilon_{0} m_{e} c^{2}\right)$ the classical electron radius, $\beta_{0}=v_{0} / c$ and $v_{0}$ the average velocity of the bunch, $\gamma_{0}=$ $\left(1-\beta_{0}^{2}\right)^{-1 / 2}$ the relativistic factor, $\sigma_{\perp}$ the transverse RMS radius, and $\sigma_{z}$ the longitudinal RMS half-length. Here, $\tilde{\epsilon}_{x}$ and $\tilde{\epsilon}_{z}$ are the longitudinal and transverse RMS geometric (or "trace-space") emittances, which are in general given by

$$
\begin{gathered}
\tilde{\boldsymbol{\epsilon}}_{x}=\sqrt{\left\langle X^{2}\right\rangle\left\langle X^{\prime 2}\right\rangle-\left\langle X X^{\prime}\right\rangle^{2}}, \\
\tilde{\boldsymbol{\epsilon}}_{z}=\sqrt{\left\langle Z^{2}\right\rangle\left\langle Z^{\prime 2}\right\rangle-\left\langle Z Z^{\prime}\right\rangle^{2}},
\end{gathered}
$$

where $X$ and $Z$ are the particle positions relative to the bunch centroid, $X^{\prime}=p_{x} / p_{0}, \quad Z^{\prime}=\delta / \gamma_{0}^{2}, \quad \delta=\left(p_{z}-\right.$ $\left.p_{0}\right) / p_{0}, p_{x}$ and $p_{z}$ are the transverse and longitudinal electron momenta, $p_{0}=m_{e} \gamma_{0} \beta_{0} c$ the average momentum of the electron bunch, $\tilde{\eta}=\beta_{0}^{2} \gamma_{0}^{2} \sqrt{\left\langle Z^{\prime 2}\right\rangle}$ is the RMS energy spread, and the angular brackets represent an ensemble average over the particles in the bunch. Within this envelope formulation, however, the emittance is assumed to be constant. Consequently, the evolution of the emittance can not be described within this envelope model and instead one of the macroparticle based methods must be used, such as the shell, the particle-in-cell, or the point-to-point model.

The quantities $A_{i}$ (see Appendix A) and $\lambda_{3}$ can be viewed as geometrical factors related to space-charge effects $[12,16,17]$, and are give by

$$
\begin{gathered}
A_{i}\left(\sigma_{\perp}, \sigma_{z}\right)=\int_{0}^{\infty} \frac{d u}{\left(\sigma_{i}^{2}+u\right) \Delta(u)} \\
\Delta(u)=\left(\sigma_{\perp}^{2}+u\right)\left(\sigma_{z}^{2}+u\right)^{1 / 2}
\end{gathered}
$$

and

$$
A=A_{1}=A_{2} .
$$

It was shown by Lapostolle et al. [18] and Sacherer et al. [16] that for ellipsoidal bunches, where the RMS emittance is either constant or specified in advance, evolution of the RMS beam projections are nearly independent of the density profile. This means that, for the calculation of the RMS dynamics, the actual distribution can be replaced by an equivalent uniform beam, which has the same RMS values. In this case, we have $\lambda_{3}=1 /(5 \sqrt{5})$.

In the shell approach, we have made use of homogeneous ellipsoidal beams to model dynamics of more general electron distributions under space-charge blowup. As a matter of consistency, it is also more convenient for the envelope description to use the radius of the uniform ellipsoid $\left(a, a_{3}\right)$ instead of RMS quantities, where $a_{i}=$ $\sqrt{5} \sigma_{i}$. The envelope equations for a bunched beam can also be rewritten to underline the transverse-longitudinal coupling through the space-charge forces [7]. Further making use of the relation [12] $2 A+A_{3}=2 /\left(a^{2} \gamma_{0} a_{3}\right)$ and writing $A_{3}$ is terms of the aspect ratio $x=a /\left(\gamma_{0} a_{3}\right)$,

$$
\begin{aligned}
& A_{3}\left(a, \gamma_{0} a_{3}\right)=\left(\gamma_{0}^{3} a_{3}^{3}\right)^{-1} g_{0}\left[a /\left(\gamma_{0} a_{3}\right)\right], \\
& g_{0}(x)=\int_{0}^{\infty}\left(x^{2}+u\right)^{-1}(1+u)^{-3 / 2} d u,
\end{aligned}
$$

allows the envelope equations to be written as

$$
a^{\prime \prime}-\frac{3}{2} \frac{N r_{e}}{\gamma_{0}^{3} \beta_{0}^{2}} \frac{1}{a a_{3}}\left[1-\frac{a^{2}}{2 \gamma_{0}^{2} a_{3}^{2}} g_{0}\left(\frac{a}{\gamma_{0} a_{3}}\right)\right]-\frac{\epsilon_{x}^{2}}{a^{3}}=0
$$

and

$$
a_{3}^{\prime \prime}-\frac{3}{2} \frac{N r_{e}}{\gamma_{0}^{5} \beta_{0}^{2}} \frac{1}{a_{3}^{2}} g_{0}\left(\frac{a}{\gamma_{0} a_{3}}\right)-\frac{\epsilon_{z}^{2}}{a_{3}^{3}}=0,
$$

where $\epsilon_{x}^{2}=5 \tilde{\epsilon}_{x}^{2}$ and $\epsilon_{z}^{2}=5 \tilde{\epsilon}_{z}^{2}$ are the transverse and longitudinal full emittances, respectively.

Section VI compares the envelope model to the other numerical methods introduced in this paper and will further define a range of application for the use of the envelope equations. In the next section, we discuss envelope models but for the specific case of beams with cylindrical shape. This specific geometry was first introduced by Chao et al. [14] to describe evolution of beams produced by plasma sources under space-charge interaction. The latter approach is more restrictive than using ellipsoids [13] because an approximate analytical derivation of the force may be obtained only for beams with small aspect ratios 
$a /\left(\gamma_{0} a_{3}\right) \ll 1$. Vacuum propagation of space-charge dominated beams often leads to aspect ratios that increase and may greatly exceed unity. The requirement of small aspect ratio however may not introduce much error if the space-charge interaction is orders of magnitude smaller when $a /\left(\gamma_{0} a_{3}\right) \sim 1$. Note also that this method cannot be used to describe nonrelativistic beams with pancake shape. The derivation of the force is tedious and is shown in Appendix E for the specific configuration discussed by Chao et al. [14], i.e., a cylindrical beam with a uniform transverse and quadratic longitudinal density profile.

\section{B. Coupled envelope equations in cylindrical geometry}

The use of envelope equations is limited to the assumptions of small energy spread (up to $\sim 50 \%$ ) and divergence (few tens of mrads) [13,14]. As mentioned above, the paper by Chao et al. [14] studied the limited case of beams with small aspect ratios $a /\left(\gamma_{0} a_{3}\right) \ll 1$, assuming a bunch with cylindrical symmetry and a uniform radial and quadratic longitudinal line-charge density profile of the form

$$
\lambda(z)=\frac{3 N}{4 a_{3}^{3}}\left(a_{3}^{2}-z^{2}\right), \quad \text { for }|z| \leq a_{3} .
$$

Chao et al. [14] used the envelope equations

$$
a^{\prime \prime}-\frac{3}{2} \frac{N r_{e}}{\gamma_{0}^{3} \beta_{0}^{2}} \frac{1}{a a_{3}}-\frac{\epsilon_{x}^{2}}{a^{3}}=0,
$$

where the transverse envelope equation is equivalent to the Kapchinskij-Vladimirskij (KV) equation [7]. The longitudinal space-charge coefficient $g_{1}(x)$ assumes a long beam $x=a /\left(\gamma_{0} a_{3}\right) \ll 1$ and is given by

$$
g_{1}(x)=2\left[\ln \left(\frac{\sqrt{5 x^{2}+1}}{\sqrt{5} x}\right)+\frac{1}{2}\right]
$$

Figure 1 shows the ratio $g_{0}(x) / g_{1}(x)$ as a function of the aspect ratio $x=a /\left(\gamma_{0} a_{3}\right)$. These two models, beside the fact that the geometry is different (ellipsoids versus cylinders), exhibit similar behaviors. Basically there is a quali-

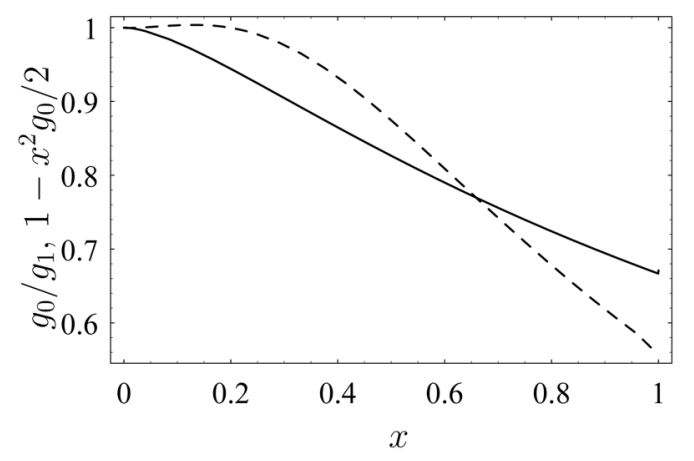

FIG. 1. Ratio of longitudinal forces $g_{0}(x) / g_{1}(x)$ (dashed line) and transverse forces $1-x^{2} g_{0}(x) / 2$ (solid line) are shown as a function of the aspect ratio $x=a /\left(\gamma_{0} a_{3}\right)$. tatively good agreement for a range $0<x<0.1$ and within $20 \%$ up to $x<0.5$.

However, further comparing both derivations of the space-charge force, in the case of a cylindrical charge distribution, showed a few conceptual differences (which are summarized in Appendix E). The expression we found for the fields in the interior of a cylinder, in the "long beam limit" approximation $(x \ll 1)$ and for $|z|<a_{3}$, using a quadratic density profile [Eq. (31)], is

$$
E_{z}=-\frac{\lambda^{\prime}(z)}{2 \pi \epsilon_{0} \gamma^{2}}\left[\ln \left(\frac{2 \gamma \sqrt{a_{3}^{2}-z^{2}}}{a}\right)-\frac{1}{2}\left(1+\frac{r^{2}}{a^{2}}\right)\right],
$$

and

$$
E_{r}=\frac{\lambda(z) r}{2 \pi \epsilon_{0} a^{2}}
$$

leading to a set of coupled envelope equations,

$$
a^{\prime \prime}-\frac{6}{5} \frac{N r_{e}}{\gamma_{0}^{3} \beta_{0}^{2}} \frac{1}{a a_{3}}-\frac{\epsilon_{x}^{2}}{a^{3}}=0
$$

and

$$
a_{3}^{\prime \prime}-\frac{3}{2} \frac{N r_{e}}{\gamma_{0}^{5} \beta_{0}^{2}} \frac{1}{a_{3}^{2}} g_{2}\left(\frac{a}{\gamma_{0} a_{3}}\right)-\frac{\epsilon_{z}^{2}}{a_{3}^{3}}=0
$$

where

$$
g_{2}(x)=-2 \ln \left(\frac{x}{\alpha_{3}}\right)
$$

and

$$
\alpha_{3} \simeq 0.6723
$$

As mentioned in the previous section, the weaknesses of using cylinders comes from the limiting assumptions that are required for an analytical expression for the fields. Assuming $x \ll 1$ allows only for an accurate description of space-charge blowup over short distances (typically on the order of $25-50 \mathrm{~mm}$ from the source for the specific case studied in [14]), as can be seen in Fig. 2 for example.

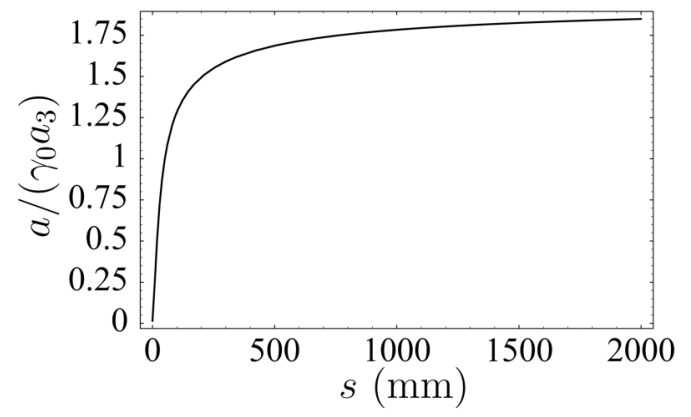

FIG. 2. Aspect ratio $x=a /\left(\gamma_{0} a_{3}\right)$ versus propagation distance $s(\mathrm{~mm})$ for an initial transverse radius $a=19 \mu \mathrm{m}$, longitudinal half-length $a_{3}=77 \mu \mathrm{m}$, divergence $x^{\prime}=2.5 \mathrm{mrad}$, energy spread $\eta=42.8 \%$ and beam relativistic factor $\gamma_{0}=13.7$. 
A direct comparison of the two models (constant density ellipsoids and parabolic cylinders) is impossible. While Eq. (29) converges to the KV equation in the limit $\gamma a_{3} \gg a$, Eq. (37) does not (a cylinder with constant longitudinal density profile would lead to the KV equation).

\section{MODIFIED ELECTROSTATIC PARTICLE-IN- CELL CODE}

In this section we describe an electrostatic particle-incell (PIC) code that may be used to model large energy spread beams. The latter characterizes an extension of the shell model (Sec. II) and does not make any assumptions on the spatial symmetries of the electron beam. The PIC result is obtained from a 3D Poisson solver [19] that calculates the electrostatic field of an arbitrary charge distribution in its rest frame. The resulting field is then Lorentz transformed to the laboratory frame. The large energy spread beam is handled through binning in momentum following Eq. (6). This method is based on the assumption of a rest frame for the beam that neglects transverse currents effects and, hence, the longitudinal magnetic field $B_{z}$. The total force per unit charge, as in Eq. (19), can be written in the form

$$
\begin{gathered}
F_{x}=\sum_{k}\left(1-\beta_{z} \beta_{k}\right) E_{k}^{(x)}, \\
F_{y}=\sum_{k}\left(1-\beta_{z} \beta_{k}\right) E_{k}^{(y)}, \\
F_{z}=\sum_{k} E_{k}^{(z)}+\beta_{x} \sum_{k} \beta_{k} E_{k}^{(x)}+\beta_{y} \sum_{k} \beta_{k} E_{k}^{(y)},
\end{gathered}
$$

where $\mathbf{E}_{k}=\left(E_{k}^{(x)}, E_{k}^{(y)}, E_{k}^{(z)}\right)$ is the electrostatic field in the laboratory frame of the $k$ th bin. This PIC method will be compared to other approaches in Sec. VI.

\section{POINT-TO-POINT INTERACTION MODEL}

This section describes the basic set of equations for a 3D point-to-point interaction model (PPI). This method allows for the calculation of space-charge dominated electron beam dynamics without the requirement of paraxial approximation, as in the previous models [Secs. II, III, and IV]. In the PPI approach, the 6D beam distribution function is also represented numerically as a collection of macroparticles. The electromagnetic fields generated by this method are calculated directly from relativistic particleparticle interaction, outlined in Ref. [15]. Radiation effects are not included and retardation effects are approximated.

Evaluation of the fields is based on a Lorentz transformation and assumes, at each time step, a constant velocity for the electrons. This assumption implies that the change in velocity must remain small (moderate space-charge interaction) during the time interval $\Delta t=$
$t-t_{\mathrm{RET}}$ where $t$ is the observation time (i.e., associated with the force evaluation) and $t_{\mathrm{RET}}$ the retarded time, i.e., the time when the macroparticle $j$ emitted the electromagnetic field seen at $t$ by macroparticle $i$. The retarded time is defined as follows:

$$
t_{\mathrm{RET}}=t-\left|\mathbf{r}-\mathbf{r}_{j}\left(t_{\mathrm{RET}}\right)\right| / c,
$$

where $\mathbf{r}$ is the observation position vector and $\mathbf{r}_{j}$ is the position vector of the $j$ th particle. Further details on how the retarded time is approximated in the PPI model is discussed in the following section.

To calculate the fields generated by particle $j$ at the position of particle $i$, first both particle coordinates are transformed to the rest frame of particle $j$,

$$
\mathbf{r}_{i j}^{\prime}=\mathbf{r}_{i j}+\frac{\gamma_{j}^{2}}{\gamma_{j}+1}\left(\mathbf{r}_{i j} \cdot \beta_{j}\right) \beta_{j},
$$

where $\mathbf{r}_{i j}=\mathbf{r}_{i}-\mathbf{r}_{j}$ is the particle separation measured in the laboratory frame and $\mathbf{r}_{i j}^{\prime}$ is the distance in the rest frame. Within the rest frame of particle $j$ only an electric field is present. This Coulomb field is given by

$$
\mathbf{E}_{j \rightarrow i}^{\prime}=\frac{Q_{m}}{4 \pi \epsilon_{0}} \frac{\mathbf{r}_{i j}^{\prime}}{\left|\mathbf{r}_{i j}^{\prime}\right|^{3}},
$$

where $Q_{m}=-N_{m} e$ is the charge of the macroparticle. Transforming this electric field back to the laboratory frame and summing over all particles yields the electromagnetic fields at the position of particle $i$,

$$
\begin{gathered}
\mathbf{E}_{i}=\sum_{j \neq i} \gamma_{j}\left\{\mathbf{E}_{j \rightarrow i}^{\prime}-\frac{\gamma_{j}}{\gamma_{j}+1}\left(\mathbf{E}_{j \rightarrow i}^{\prime} \cdot \beta_{j}\right) \beta_{j}\right\}, \\
c \mathbf{B}_{i}=\sum_{j \neq i} \gamma_{j} \beta_{j} \times \mathbf{E}_{j \rightarrow i}^{\prime} .
\end{gathered}
$$

Each macroparticle represents a large number $N_{m}$ of elementary electrons. In order to reduce the large angle scattering effect, which overestimates the real collision cross section in PPI models, the macroparticles should be considered as particle clouds of radius $r_{0}$. Under this assumption, within the cloud, the Coulomb repulsion force decreases to zero when two clouds completely overlap, that is

$$
\mathbf{E}_{j \rightarrow i}^{\prime}=\frac{Q_{m}}{4 \pi \epsilon_{0}} \frac{\mathbf{r}_{i j}^{\prime}}{r_{0}^{3}}, \quad \text { if }\left|\mathbf{r}_{i j}^{\prime}\right|<r_{0} .
$$

The primary approximations used in the PPI model are the neglection of radiation, an approximation of the retarded time, the use of a finite number of macroparticles, each representing $N_{m}$ electrons, and the introduction of the parameter $r_{0}$. The comparisons presented in the following sections indicate that the PPI model yields the most accurate results for large energy spread beams compared to the two other methods (shells or PIC). 


\section{A. Benchmarking}

The benchmarking of the PPI algorithm has been done by simulating the blowup of an initially cold homogeneous spherical electron distribution. This test problem was chosen since there exists an exact analytical calculation of the initial total energy of the system. The energy conservation law for a system of $N_{m}$ macroparticles interacting with an electromagnetic field is given by [20]

$$
\begin{aligned}
\frac{\partial}{\partial t}[ & \left.\sum_{i}^{N_{m}}\left(\gamma_{i}-1\right) m_{e} c^{2}+\int_{V} d^{3} x\left(\frac{\epsilon_{0} \mathbf{E}^{2}}{2}+\frac{\mathbf{B}^{2}}{2 \mu_{0}}\right)\right] \\
& +\oint_{S} d S \mathbf{n} \cdot \mathbf{S}=0,
\end{aligned}
$$

where $\mathbf{S}$ is the Poynting vector, $V$ is a volume containing the entire charged particle system, $S$ the corresponding surface enclosing the volume $V$, and $\mathbf{n}$ a unit vector normal (outward) to the surface $S$. The electron distribution is taken initially cold, which implies that the total energy at $t=0$ is purely electrostatic (assuming radiation fields are negligible), that is

$$
H_{i}=\frac{N_{m} U_{0}}{N_{e}}
$$

together with

$$
U_{0}=\int_{V} d^{3} x \frac{\epsilon_{0} \mathbf{E}^{2}}{2}=\frac{3 Q^{2}}{20 \pi R \epsilon_{0}},
$$

where integration is over all space and $N_{e}$ is the total number of real electrons. In deriving Eq. (52), the expression of the electrostatic field of a spherical homogeneous charge distribution $\mathbf{E}=\rho r /\left(3 \epsilon_{0}\right)$ has been used along with the charge density $\rho=3 Q /\left(4 \pi R^{3}\right)$. With the initial condition of a sphere of charge $Q$ and radius $R$, the PPI simulation is run until the space-charge interaction is negligible and the electron motion is purely ballistic. At the final time step, the expression for the total energy becomes

$$
H_{f}=\sum_{i}^{N_{m}}\left(\gamma_{i}-1\right) m_{e} c^{2} .
$$

Energy conservation requires $\Delta H=\left|H_{f}-H_{i}\right|=0$.

Figure 3 shows the time evolution of the equivalent uniform-density bunch with dimensions $a_{1}, a_{2}$, and $a_{3}$ (the equivalent uniform-density quantities are obtained from the RMS values by multiplication by $\sqrt{5}$ ), and the RMS normalized momentum $\Delta u_{x}, \Delta u_{y}$, and $\Delta u_{z}$, for the following initial beam parameters: charge $Q=1 \mathrm{nC}$, radius $R=a_{1}=a_{2}=a_{3}=24 \mu \mathrm{m}$, average energy $E_{0}=$ 0 , energy spread $\eta=0$, and divergence $x_{\max }^{\prime}=0$. An identical time evolution of the beam RMS dynamics along each of the three axes $(O x, O y, O z)$ is found as expected.

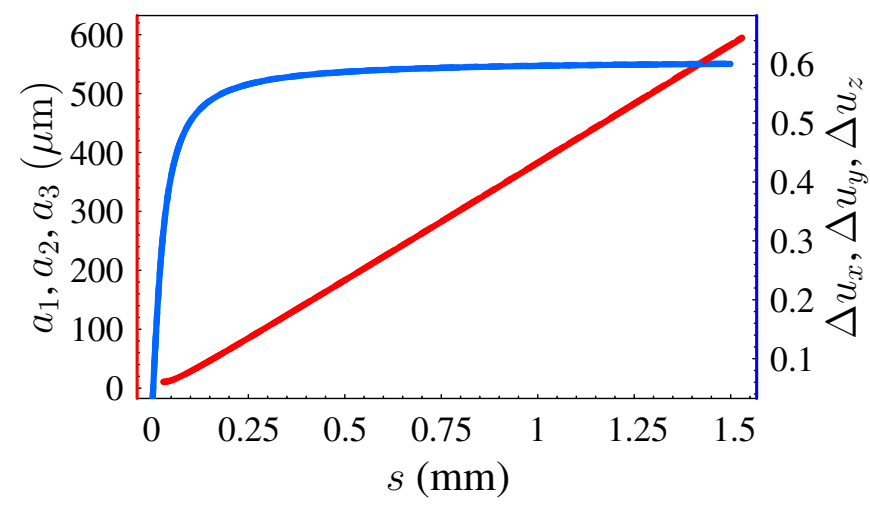

FIG. 3. (Color) Simulation of the space-charge blowup of an initially cold spherical electron distribution with parameters: Charge $Q=1 \mathrm{nC}$, beam energy $E_{0}=0$, radius $a_{1}=a_{2}=a_{3}=$ $24 \mu \mathrm{m}$, energy spread $\eta=0$, divergence $x_{\max }^{\prime}=0$, average distance between macroparticles $n_{0}^{-1 / 3} \simeq 1.74 \mu \mathrm{m}$, and cutoff radius $r_{0}=100 \mathrm{~nm}$. Beam radius and RMS momentum spread along the three coordinate axes are shown as a function of propagation distance $s$.

The corresponding relative error in the energy conservation is shown in Fig. 4(a), for various sizes $r_{0}$, where $\Gamma=$ $\left(H_{f}-H_{i}\right) /\left(H_{f}+H_{i}\right)$. An estimate of the typical distance between macroparticles in the system $d=n_{0}^{-1 / 3}$ has been introduced through the initial macroparticle density $n_{0}$, that is

$$
n_{0}=\frac{3 N_{m}}{4 \pi a^{2} a_{3}}
$$

where $N_{m}=\left|Q_{m} / e\right|$ is the number of macroparticles. As can be seen in Fig. 4(a), choosing $r_{0}>n_{0}^{-1 / 3}$ introduces an excessive smoothing of the electromagnetic fields leading to a deviation from energy conservation.

Figure 4(b) plots the energy conservation relative error for the case of a moving ellipsoidal charge distribution with identical initial parameters as Fig. 4(a) except for $E_{0}=1.12 \mathrm{MeV}$ and $a_{3}=10.92 \mu \mathrm{m}$, corresponding to a cold spherical electron distribution in its rest frame, i.e., $a_{1}^{\prime}=a_{2}^{\prime}=a_{3}^{\prime}=24 \mu \mathrm{m}\left(a_{3}^{\prime}=\gamma_{0} a_{3}\right)$. The initial total energy of the system can be calculated analytically, i.e., according to relativistic principles, any quantity of rest energy $W^{0}$ will be attributed the value $\gamma_{0} W^{0}$ by an observer who sees it being transported with a velocity $v_{0}=c(1-$ $\left.\gamma_{0}^{-2}\right)^{-1 / 2}$ [21]. The above calculations for a spherical charge distribution in the beam rest frame may thus be generalized to any arbitrary inertial frame, giving an expression for the total energy (kinetic plus potential)

$$
H_{i}=N_{m}\left(\gamma_{0}-1\right) m_{e} c^{2}+\left(\frac{N_{m}}{N_{e}}\right) \gamma_{0} U_{0} .
$$

Figures 4(a) and 4(b) show a similar behavior, that is, for $r_{0}<n_{0}^{-1 / 3}$ the PPI method reaches convergence. The rela- 

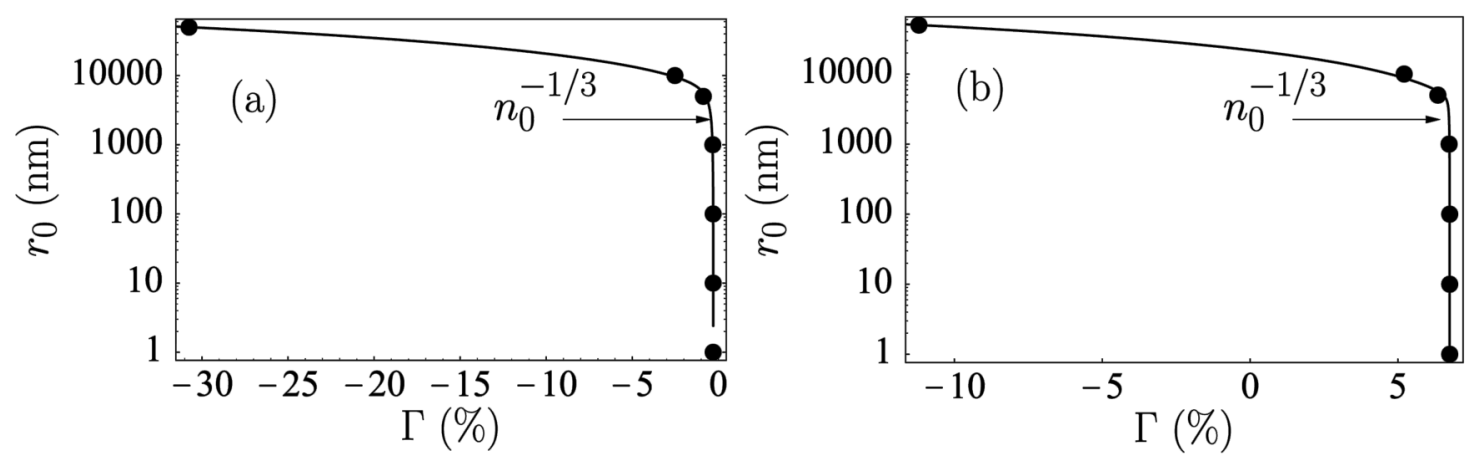

FIG. 4. Energy conservation relative error $\Gamma$ versus cutoff parameter $r_{0}$ for (a) an initially cold spherical electron distribution with parameters: Charge $Q=1 \mathrm{nC}$, beam energy $E_{0}=0$, radius $a_{1}=a_{2}=a_{3}=24 \mu \mathrm{m}$, energy spread $\eta=0$, divergence $x_{\text {max }}^{\prime}=0$, typical distance between macroparticles $n_{0}^{-1 / 3} \simeq 1.74 \mu \mathrm{m}$, and (b) a moving ellipsoidal charge distribution with identical parameters except for $E_{0}=1.12 \mathrm{MeV}$ and $a_{3}=10.92 \mu \mathrm{m}$, corresponding to a cold spherical electron distribution in its rest frame, i.e., $a_{1}^{\prime}=$ $a_{2}^{\prime}=a_{3}^{\prime}=24 \mu \mathrm{m}\left(a_{3}^{\prime}=\gamma_{0} a_{3}\right)$. The latter allows for a direct comparison with analytical estimates.

tive error is found to be less than $-5 \times 10^{-2} \%$ for the case of Fig. 4(a), providing an accurate calculation of beam dynamics, and within $6.75 \%$ for Fig. 4(b). Those two particular examples are collisionless, indicating that there is no additional requirement on $r_{0}$ beside the condition $r_{0}<n_{0}^{-1 / 3}$. For systems where collisions may play an important role, the relative error in the energy conservation is expected to remain unchanged for $r_{0}<n_{0}^{-1 / 3}$ because the collisions are purely elastic. To get a correct estimate for the minimum value of $r_{0}$, conservation of phase-space structure may be considered as an alternate means. For these systems, $r_{0} \sim n_{0}^{-1 / 3}$ is considered best.

The difference in the relative error shown in Fig. 4 may be explained using geometrical arguments and is mainly caused by the approximate way retardation effects are handled in the PPI method. Figure 5 shows a simplified configuration of two macroparticles, which is sufficient to describe the problem because the PPI method calculates the interaction force between two macroparticles at a time (N-body). Obviously this is not the case for the other methods introduced in this paper (the shell approach, for instance, smooths the macroparticle distribution). The PPI method uses the position of the macroparticle and the point of observation at the present time to calculate the fields. As explained earlier, a Lorentz transformation to the frame comoving with the macroparticle is performed and the distance $r^{\prime}\left(t^{\prime}=0\right)$ [Fig. 5(b)] is used to evaluate the electrostatic field in the rest frame (the macroparticle is static). This approach does not neglect retardation but instead performs an approximate evaluation. Actually, the Lorentz transform yields the exact retarded time for a particle moving at a constant velocity. This can be illustrated by considering a macroparticle that has a constant velocity in the lab frame. Then, in the rest frame, its location remains at the origin at all times $t^{\prime}$ (the location
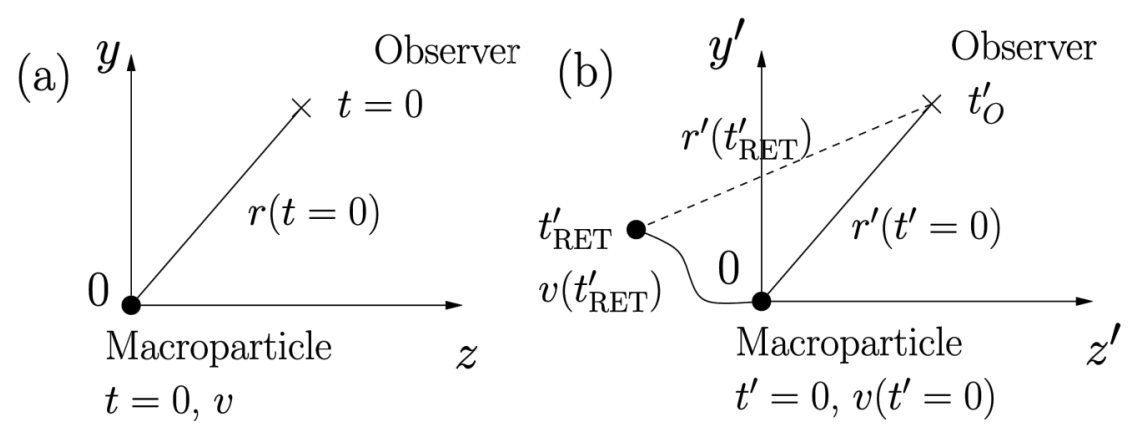

FIG. 5. (a) Two macroparticles in the laboratory frame are shown. Their respective positions are shown at the present time; consistent with the assumptions in the PPI method. (b) Corresponding two macroparticles in the frame comoving with the macroparticle located at the origin (we discuss the electromagnetic fields emitted by the latter and seen by the observer, assumed to be located at the position of the second macroparticle). $r^{\prime}\left(t^{\prime}=0\right)$ is the actual distance used to calculate the force in the PPI method; the latter would be exact if the macroparticle had a constant velocity during the time interval it takes the light to travel from the macroparticle to the point of observation. For the case of a velocity spread the macroparticle was located at another position than the origin at $t^{\prime}=t_{\mathrm{RET}}^{\prime}$, where $t_{\mathrm{RET}}^{\prime}$ is the retarded time. This is the error introduced in the PPI method, explained using geometrical arguments. Note that retardation effects are not neglected in the PPI method but simply approximated as explained above. 
of the macroparticle is chosen to be at the origin for convenience). In this particular case, the position $r^{\prime}\left(t^{\prime}=\right.$ 0 ) remains valid even at the retarded time $t^{\prime}=t_{\mathrm{RET}}^{\prime}$, which is the actual time the observer sees the electromagnetic field emitted by the macroparticle. In other words, in this case the electromagnetic field calculated in the lab frame is exact and includes retardation. Furthermore, radiation is absent because the velocity is constant.

In actuality, however, the macroparticle will experience acceleration due to the space-charge force of the surrounding particles and, hence, the above Lorentz transform method no longer yields the exact retarded time. This error in the retarded time will be small if the acceleration is small. Specifically, if during the time interval $\Delta t^{\prime}=t_{0}^{\prime}-$ $t_{\mathrm{RET}}^{\prime}=\left|r^{\prime}\left(t_{\mathrm{RET}}^{\prime}\right)\right| / c$ (in the rest frame), the change in velocity is small, $\quad \Delta v^{\prime} / v\left(t_{\mathrm{RET}}^{\prime}\right)=\left[v\left(t_{\mathrm{RET}}^{\prime}\right)-v\left(t^{\prime}=\right.\right.$ $0)] / v\left(t_{\mathrm{RET}}^{\prime}\right) \ll 1$, then retardation effects are approximately correct, i.e., $\Delta r^{\prime} / r^{\prime}\left(t_{\mathrm{RET}}^{\prime}\right)=\mid r^{\prime}\left(t_{\mathrm{RET}}^{\prime}\right)-r^{\prime}\left(t^{\prime}=\right.$ $0) \mid / r^{\prime}\left(t_{\text {RET }}^{\prime}\right) \ll 1$ (see Fig. 5).

Using the above argument, the difference in the relative error found in Fig. 4 between the spherical charge distributions at rest or moving with $\gamma_{0} m_{e} c^{2} \simeq 1.12 \mathrm{MeV}$ may be explained as follows. Comparing two identical events in the beam frame (for example the space-charge interaction between two macroparticles at the same time $t^{\prime}$ in both cases), the distance $\Delta r^{\prime}$ will artificially change for the moving bunch introducing a greater error $\Delta r^{\prime} / r^{\prime}\left(t_{\mathrm{RET}}^{\prime}\right)$. For ultrarelativistic beams however $\left(\gamma_{0} \gg 1\right), \Delta r^{\prime}$ will reach an asymptotic value, leading to a constant error in the evaluation of the fields.

\section{SIMULATION RESULTS}

In this section, the different analytical and numerical methods introduced in this paper will be compared and ranges of applicability for each method will be determined.
These methods will be further used for the simulation of vacuum transport of electron beams produced by plasma sources.

\section{A. Compact electron sources}

\section{Small energy spread}

The validity of the envelope approach requires small divergence (up to a few tens of mrads), low peak current, and low energy spread ( $\eta \lesssim 50 \%$ ). In Fig. 6 , we plot the equivalent uniform-density bunch radius and bunch length as a function of propagation distance using, respectively, the shell approach, the coupled envelope equations (29) and (30), and the 3D-PPI code. The bunch charge is $Q=$ $100 \mathrm{pC}$, the initial radius $a=6 \mu \mathrm{m}$, length $a_{3}=2.5 \mu \mathrm{m}$, divergence $x^{\prime}=2 \mathrm{mrad}$, energy spread $\eta=5 \%$, average beam energy $E_{0}=5.25 \mathrm{MeV}$, aspect ratio $x=$ $a /\left(\gamma_{0} a_{3}\right) \simeq 0.23$, and cutoff radius $r_{0}=100 \mathrm{~nm}$. Good agreement is found between the three models, within a $4.8 \%$ margin. For such beams the coupled envelope equations can be applied accurately [13]. This typical example is also found to be an upper limit for the usability of either the shell or the envelope models and corresponds to a maximum current given by

$$
\bar{I}=\bar{I}_{\max }=\frac{3 Q}{4 a_{3}} \beta_{0} c \simeq 9 \mathrm{kA} .
$$

It is possible to define a general quantity for the beam in order to better characterize the space-charge blowup and also the range of applicability of either the shell method or the envelope equations. It can be expressed as the ratio of the beam current normalized with respect to the Alfvèn current, that is

$$
\alpha_{b}=\frac{\bar{I}}{\gamma_{0} I_{A}} \frac{1.8 \times 10^{2}}{a_{[\mu \mathrm{m}]}^{2}},
$$
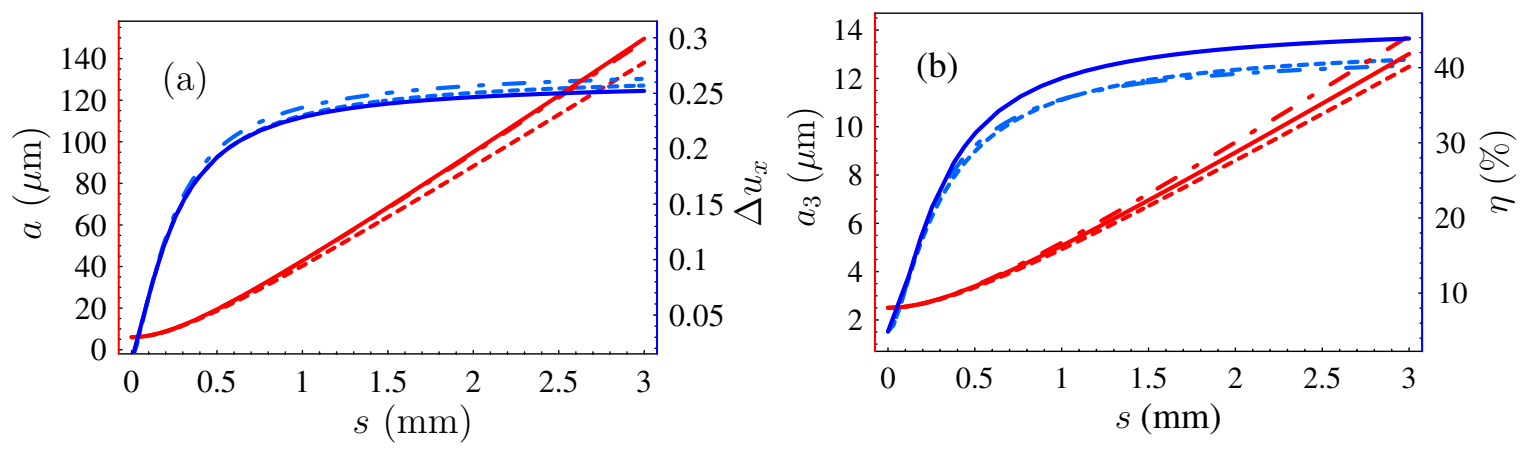

FIG. 6. (Color) Simulation of the space-charge blowup of a small energy spread compact electron bunch with input parameters: Charge $Q=100 \mathrm{pC}$, beam energy $E_{0}=5.25 \mathrm{MeV}$, radius $a=6 \mu \mathrm{m}$, half-length $a_{3}=2.5 \mu \mathrm{m}$, energy spread $\eta=4.8 \%$, divergence $x_{\max }^{\prime}=2 \mathrm{mrad}$, typical distance between macroparticles $n_{0}^{-1 / 3} \simeq 266 \mathrm{~nm}$, and cutoff radius $r_{0}=100 \mathrm{~nm}$. Evolution of (a) transverse radius $a=\sqrt{5} \sigma_{x}$ and transverse momentum spread $\Delta u_{x}$, (b) longitudinal half-length $a_{3}=\sqrt{5} \sigma_{z}$ and energy spread $\eta=$ $\sqrt{5} \Delta u_{z} /\left\langle u_{z}\right\rangle$ as a function of propagation distance $s=c t$ is shown using the envelope model (solid), 3D-PPI (dashed), and shell method (dot-dashed). 

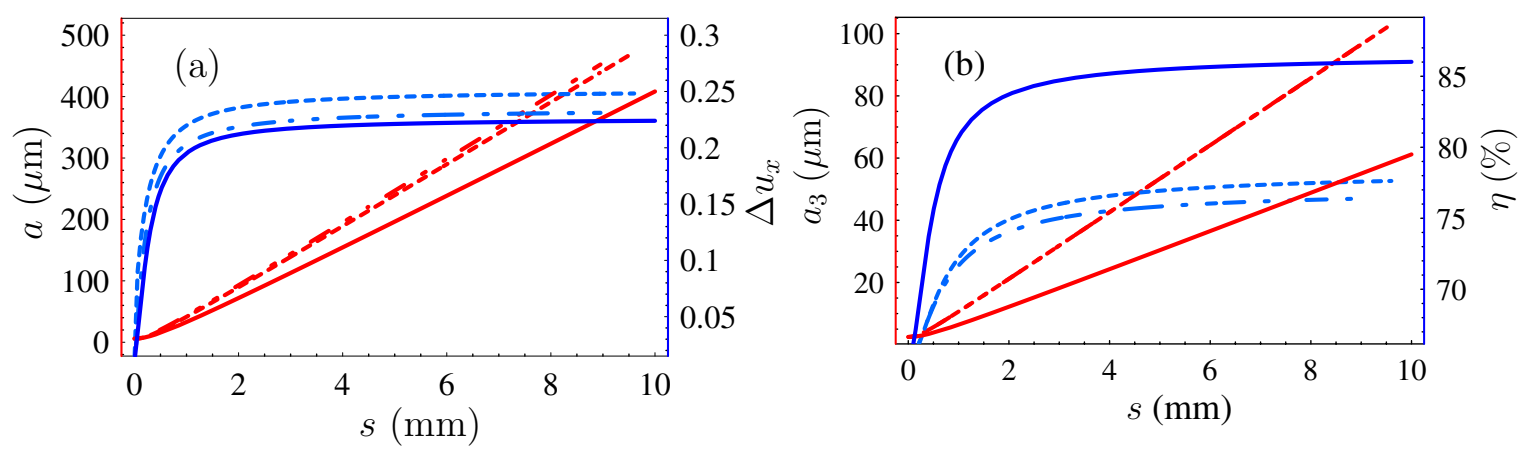

FIG. 7. (Color) Simulation of the space-charge blowup of a large energy spread compact electron bunch with input parameters: charge $Q=100 \mathrm{pC}$, beam energy $E_{0}=6 \mathrm{MeV}$, transverse radius $a=6 \mu \mathrm{m}$, half-length $a_{3}=2.5 \mu \mathrm{m}$, energy spread $\eta=65 \%$, divergence $x_{\max }^{\prime}=2 \mathrm{mrad}$, typical distance between macroparticles $n_{0}^{-1 / 3} \simeq 266 \mathrm{~nm}$, and cutoff radius $r_{0}=100 \mathrm{~nm}$. Evolution of (a) transverse radius $a$ and transverse RMS momentum spread $\Delta u_{x}$, (b) longitudinal half-length $a_{3}$ and energy spread $\eta$ as a function of propagation distance $s$ is shown using the envelope model (solid), 3D-PPI (dashed), and shell method (dot-dashed).

where $I_{A}=17 \times 10^{3} \gamma_{0} \beta_{0}$. The ratio $\alpha_{b}$ approximately characterizes the initial averaged magnitude of the spacecharge force assuming a "long" beam under the paraxial approximation, i.e., when transverse current is negligible. For $\bar{I}=\bar{I}_{\max }, \alpha_{b} \simeq 1$ and in general $\alpha_{b} \leq 1$ is necessary for using the envelope and shell description.

\section{Large energy spread}

For beams with large energy spread, Eqs. (29) and (30) do not apply anymore but the shell model is found to be a very accurate method. Figure 7 plots the uniform-density bunch radius $a$, bunch length $a_{3}$, energy spread $\eta$, and the RMS transverse normalized momentum $\Delta u_{x}$ as a function of propagation distance $s$ and for the three models, i.e., envelope, shell, and PPI. The electron bunch energy is $E_{0}=6 \mathrm{MeV}$, the initial bunch radius $a=6 \mu \mathrm{m}$, bunch length $a_{3}=2.5 \mu \mathrm{m}$, divergence $x^{\prime}=2 \mathrm{mrad}$, energy spread $\eta=65 \%$, and total charge $Q=100 \mathrm{pC}$.

Figure 8 shows the same quantities, i.e., $a(s), a_{3}(s)$, $\Delta u_{x}(s)$, and $\eta(s)$, but for a long bunch. The electron energy is $E_{0}=8.5 \mathrm{MeV}$, the initial bunch radius $a=19 \mu \mathrm{m}$, bunch length $a_{3}=76.8 \mu \mathrm{m}$, divergence $x^{\prime}=3 \mathrm{mrad}$, energy spread $\eta=74.5 \%$, and total charge $Q=1.6 \mathrm{nC}$. In this case the energy spread $\eta(s)$ exhibits a typical behavior that is a direct contribution of the terms $\beta_{x} B_{y}-\beta_{y} B_{x}$ in the longitudinal force. These terms are naturally neglected in the envelope equation, under the paraxial approximation, but included in the shell and PPI. From Figs. 7 and 8, it is found that the shell and PPI codes provide a similar description of the RMS bunch dynamics within an $4 \%$ margin whereas the difference with respect to the coupled envelope equations is more than $27 \%$ relative error.

\section{B. Application to plasma sources 1. Colliding pulse injection}

Test particle simulations of the colliding pulse LWFA injector [2-5], in which two counterpropagating laser pulses are used to inject electrons from the background plasma directly into the wake, indicate the production of a trapped bunch with $N \sim 10^{8}$ electrons, a low energy spread
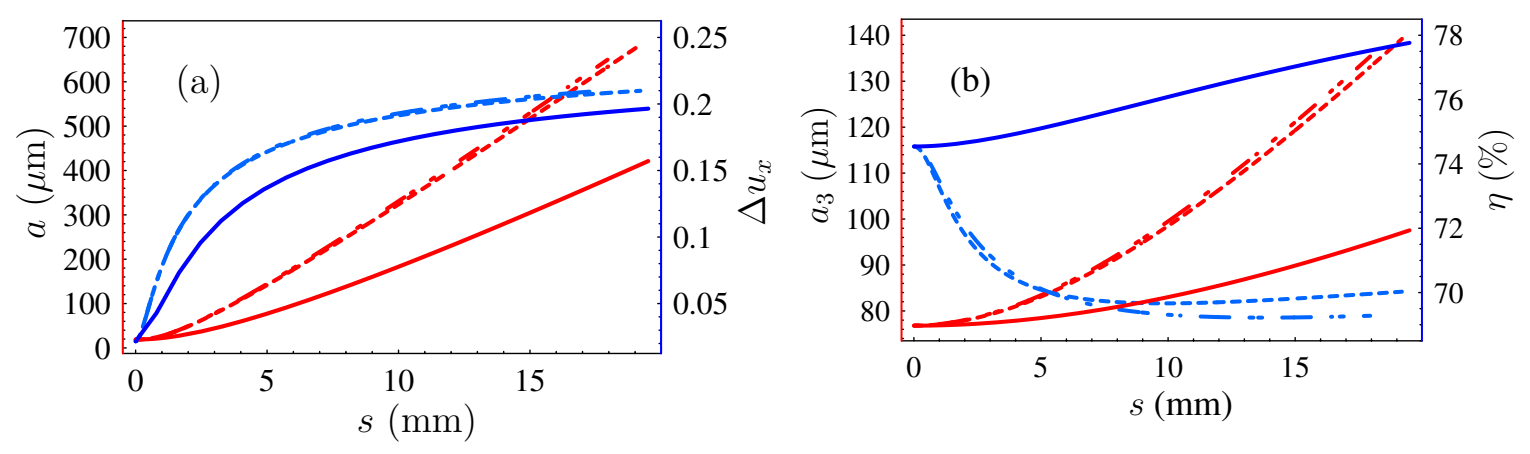

FIG. 8. (Color) Simulation of the space-charge blowup of a large energy spread long electron bunch with input parameters: Charge $Q=1.6 \mathrm{nC}$, beam energy $E_{0}=8.5 \mathrm{MeV}$, radius $a=19 \mu \mathrm{m}$, half-length $a_{3}=76.8 \mu \mathrm{m}$, energy spread $\eta=74.5 \%$, divergence $x_{\max }^{\prime}=3 \mathrm{mrad}$, typical distance between macroparticles $n_{0}^{-1 / 3} \simeq 1.8 \mu \mathrm{m}$, and cutoff radius $r_{0}=1 \mu \mathrm{m}$. Evolution of (a) transverse radius $a$ and transverse RMS momentum spread $\Delta u_{x}$, (b) longitudinal half-length $a_{3}$ and energy spread $\eta$ as a function of propagation distance $s$ is shown using the envelope model (solid), 3D-PPI (dashed), and shell method (dot-dashed). 

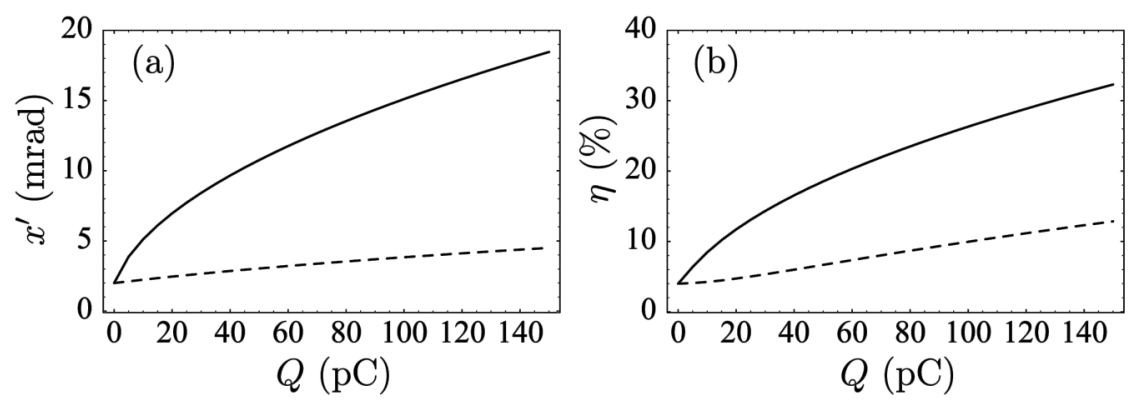

FIG. 9. Vacuum propagation of a compact electron beam produced by plasma-based accelerators using the colliding pulse injection scheme. Input parameters: Beam energy $E_{0}=15 \mathrm{MeV}$ (solid line) and $E_{0}=45 \mathrm{MeV}$ (dashed line), radius $a=6 \mu \mathrm{m}$, half-length $a_{3}=2.5 \mu \mathrm{m}$, energy spread $\eta=4 \%$, and divergence $x_{\max }^{\prime}=2 \operatorname{mrad}$. Estimates for (a) the beam divergence and (b) energy spread as a function of charge is shown for a beam located in vacuum at $s=10 \mathrm{~cm}$ from the plasma exit.

$\eta<1-5 \%$, low normalized emittance $\epsilon_{x} \sim 1 \mathrm{~mm}-\mathrm{mrad}$, a transverse size on the order of the laser spot size $\sigma_{\perp} \sim$ $6 \mu \mathrm{m}$ and of ultrashort duration, $\sigma_{z} \sim 1-5 \mu \mathrm{m}$. Such test particle simulations, however, neglected the space-charge effects of the accelerated bunch.

Space-charge effects can limit the amount of charge that can be transported in an ultrashort, tightly focused electron bunch, i.e., space charge can lead to a increase in both the longitudinal and transverse bunch dimensions. In a LWFA, space-charge effects may not be of concern while the bunch is in the plasma wave, since the longitudinal and transverse fields of the wake are typically much greater than the space-charge forces of the bunch. This is not the case, however, as the bunch exits the plasma into a vacuum region with no applied fields. In this case, space charge can lead to a rapid blowup of the bunch.

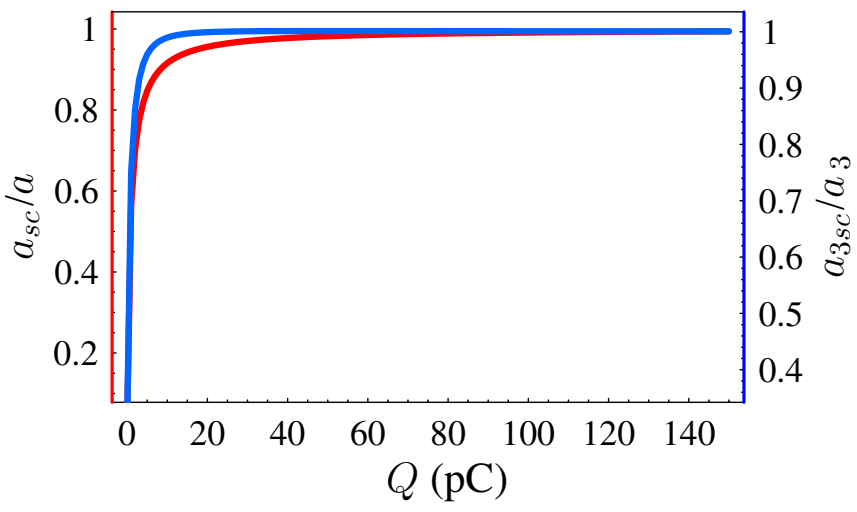

FIG. 10. (Color) Ratio of the radius $a_{s c} / a$ and half-length $a_{3 s c} / a_{3}$ as a function of charge is shown for a typical beam produced by colliding pulse injection. This calculation has been performed using the envelope model where $a_{s c}(s)$ assumes the beam is space-charge dominated. Regimes where $a_{s c} / a \ll 1$ are emittance dominated and $a_{s c} / a \simeq 1$ are space-charge dominated, respectively. Input parameters: Bunch energy $E_{0}=15 \mathrm{MeV}$, radius $a=6 \mu \mathrm{m}$, half-length $a_{3}=1.5 \mu \mathrm{m}$, energy spread $\eta=$ $4 \%$, divergence $x_{\max }^{\prime}=2 \mathrm{mrad}$, and the beam is taken to be located in vacuum, $s=10 \mathrm{~cm}$ from the plasma exit.
Figure 9 plots the bunch divergence $x^{\prime}$ and energy spread $\eta$, by solving Eqs. (29) and (30), as a function of charge $Q$ (at $s=10 \mathrm{~cm}$ ) assuming an electron bunch energy $E_{0}=$ $15 \mathrm{MeV}$ (solid line) and $E_{0}=45 \mathrm{MeV}$ (dashed line), an initial bunch radius $a=6 \mu \mathrm{m}$, bunch length $a_{3}=$ $2.5 \mu \mathrm{m}$, divergence $x^{\prime}=2 \mathrm{mrad}$, energy spread $\eta=4 \%$, and a total amount of charge from 0 to $150 \mathrm{pC}$. The beam was initially assumed to be at focus, i.e., $a^{\prime}(0)=$ $a_{3}^{\prime}(0)=0$.

Figure 10 plots the ratios $a_{s c} / a$ and $a_{3 s c} / a_{3}$ at $s=$ $10 \mathrm{~cm}$, where $a_{i}$ is the bunch radius, obtained by solving Eqs. (29) and (30) for the same parameters as Fig. 9, and $a_{s c}(s)$ is the bunch radius assuming the beam is spacecharge dominated, i.e., in this case the terms $\epsilon_{x}^{2} / a^{3}$ and $\epsilon_{z}^{2} / a_{3}^{3}$ are removed from Eqs. (29) and (30). Note that Fig. 10 allows for a clear separation of the two regimes: (i) emittance dominated $a_{s c} / a_{i} \ll 1$ and (ii) space-charge dominated $a_{s c} / a_{i} \simeq 1$.

These figures show that a fairly high energy electron bunch with a total charge of several $\mathrm{pC}$ produced by colliding pulse injection can rapidly blow up via space charge due to its very compact size. For this typical bunch, below $5 \mathrm{pC}$ the beam is emittance dominated, i.e., in this case one can neglect the effect of space charge. Above this value space charge must be considered and clearly participates in the beam growth. However, for electron beams in the energy range $\sim 45 \mathrm{MeV}$, space-charge effects will be greatly reduced. A plasma-based accelerator using the CPI scheme should operate in that regime.

\section{SM-LWFA injectors using model distributions}

The development of high intensity short laser pulses has made it possible to study high energy electron production on a tabletop. Relativistic electrons have been observed worldwide in many experiments over the past years [2232]. The common setup for these experiments was a single intense incoming laser pulse focused on a supersonic gas jet without a preformed plasma channel. The typical diameter of the gas jets ranged on the order $0.5-2 \mathrm{~mm}$. It was found that the number of electrons versus energy produced 

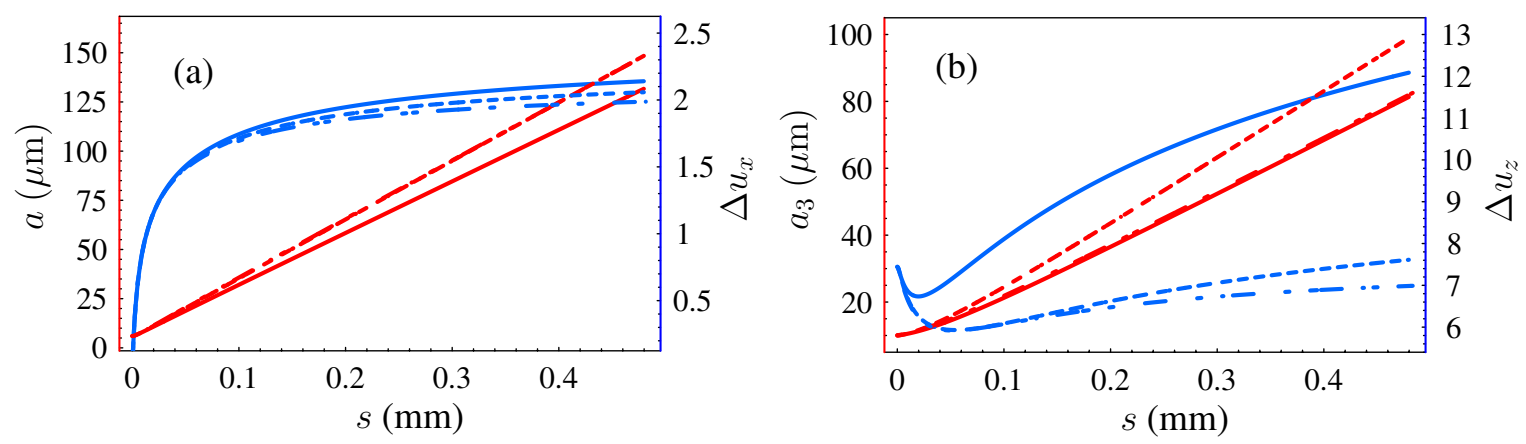

FIG. 11. (Color) Simulation of the space-charge blowup of typical beams produced by plasma accelerators in the self-modulated regime. Input parameters: Charge $Q=5 \mathrm{nC}$, beam temperature $k_{B} T=4 \mathrm{MeV}$, radius $a=6 \mu \mathrm{m}$, half-length $a_{3}=10 \mu \mathrm{m}$, divergence $x_{\max }^{\prime}=2 \mathrm{mrad}$, typical distance between macroparticles $n_{0}^{-1 / 3}=422 \mathrm{~nm}$, and cutoff radius $r_{0}=100 \mathrm{~nm}$. Evolution of (a) transverse radius $a$ and transverse RMS momentum spread $\Delta u_{x}$, (b) longitudinal half-length $a_{3}$ and longitudinal RMS momentum spread $\Delta u_{z}$ as a function of propagation distance $s$ is shown using the 3D-PPI model (solid), electrostatic PIC (dashed), and shell method (dot-dashed).

in those single-beam experiments fits either a single or a double exponential decay law $d N / d E \propto \exp \left(-E / T_{e}\right)$, where $T_{e}$ is the electron temperature that was experimentally found to be typically on the order of $4 \mathrm{MeV}$.

In Fig. 11, we plot the equivalent uniform-density bunch radius, bunch length, divergence, and relative momentum spread, as a function of propagation distance, for the SMLWFA case. The equivalent uniform-density quantities are obtained from the RMS values by multiplication by $\sqrt{5}$. The initial phase-space density was assumed uniform, with a bunch charge of $Q=5 \mathrm{nC}$. The initial bunch longitudinal momentum ranges from $p_{z 0}=0.1 \rightarrow 23 \mathrm{MeV} / c$, corresponding to an exponential distribution with $k T=$ $4 \mathrm{MeV}$ (Fig. 12). The initial bunch radius was $a=$ $6 \mu \mathrm{m}$, initial length $a_{3}=10 \mu \mathrm{m}$, and initial divergence $x_{0}^{\prime}=2$ mrad. From Fig. 11(a) we see a good agreement for the transverse RMS size among the three models: shells (dot-dashed line), PPI (solid line), and PIC (dashed line). However, the beam distributions exhibit differences and this may be an issue for beams with large energy spread, i.e., RMS quantities may not provide enough information and higher order moments may need to be included.

It is also found, from Fig. 11(a), that transverse spacecharge effects vanish at a very early stage $(\sim 200 \mu \mathrm{m})$, whereas the longitudinal dynamics are more persistent as can be seen in Fig. 11(b). This plot shows, on the contrary, disagreement between the three models. This indicates that paraxial approximation is not valid and that more general space-charge models such as PPI or fully $3 \mathrm{D}$ electromagnetic PIC codes are needed. Figure 11(b) shows clear evidence that neither the shell (Sec. II) nor the electrostatic PIC model (Sec. IV) apply anymore. Recall that the main difference between electrostatic PIC and shells comes from the smoothing of the density within an ellipsoidal shell. This explains the incapability of the shell model to describe beam dynamics when the local beam phase-space correlations play an important role.
For this particular example of an initial beam distribution with a Boltzmann energy profile, the low-energy highdensity region will experience both transverse blowup and longitudinal acceleration. The latter results in a substantial increase of the energy spread over long distances, i.e., at $s=1.5 \mathrm{~mm}$ (not shown in Fig. 11). The energy spread is a factor of 2 higher than its initial value. The longitudinal dynamics reach the ballistic state after a propagation distance of $s \sim 1.5 \mathrm{~mm}$. Note that the dynamics of the low energy region lead to a general change of the final beam profile, as shown in Fig. 12, i.e., the distribution peaks around $u_{z} \simeq 45$.

In order to investigate the validity of the paraxial approximation, the PPI algorithm is modified so as to include the latter assumption, i.e., the transverse normalized velocities in the field solver are neglected $\beta_{x j}=\beta_{y j}=0$ and $\beta_{j}=\beta_{z j} \mathbf{e}_{z}$, where $\mathbf{e}_{z}$ is the axial unit vector. Equations (47) and (48) become

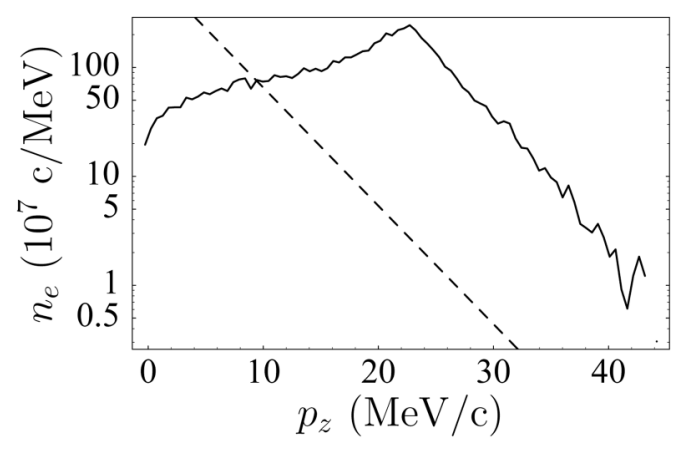

FIG. 12. Electron bunch energy density for the same initial parameters as Fig. 11, that is, total beam charge $Q=5 \mathrm{nC}$, temperature $k_{B} T=4 \mathrm{MeV}$, radius $a=6 \mu \mathrm{m}$, half-length $a_{3}=$ $10 \mu \mathrm{m}$, divergence $x_{\max }^{\prime}=2 \mathrm{mrad}$, average distance between macroparticles $n_{0}^{-1 / 3}=422 \mathrm{~nm}$, and cutoff radius $r_{0}=100 \mathrm{~nm}$. Part (a) shows the input electron distribution and (b) after a vacuum propagation of $s=1.5 \mathrm{~mm}$. 

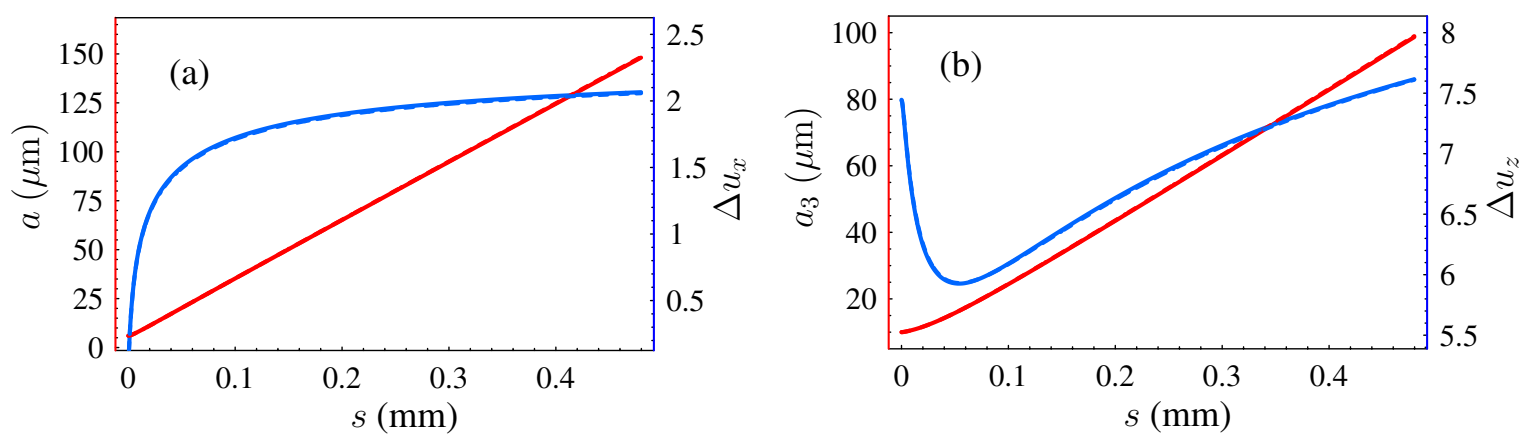

FIG. 13. (Color) Comparison between the reduced 3D-PPI model in the paraxial approximation (solid line) and the electrostatic PIC (dashed line) for the case of a large energy spread beam with an exponential energy profile. Input parameters: Charge $Q=5$ nC, temperature $k_{B} T=4 \mathrm{MeV}$, radius $a=6 \mu \mathrm{m}$, half-length $a_{3}=10 \mu \mathrm{m}$, divergence $x_{\max }^{\prime}=2 \mathrm{mrad}$, typical distance between macroparticles $n_{0}^{-1 / 3}=422 \mathrm{~nm}$ and cutoff radius $r_{0}=100 \mathrm{~nm}$. Evolution of (a) transverse radius $a$ and transverse RMS momentum spread $\Delta u_{x}$, (b) longitudinal half-length $a_{3}$ and longitudinal RMS momentum spread $\Delta u_{z}$ as a function of propagation distance $s$ is shown.

$$
\begin{gathered}
\mathbf{E}_{\perp i}=\sum_{j \neq i} \gamma_{j} \mathbf{E}_{\perp, j \rightarrow i}^{\prime}, \\
\mathbf{E}_{z i}=\sum_{j \neq i} \mathbf{E}_{z, j \rightarrow i}^{\prime}, \\
c \mathbf{B}_{i}=\sum_{j \neq i} \gamma_{j} \beta_{z j} \mathbf{e}_{z} \times \mathbf{E}_{i},
\end{gathered}
$$

where $\mathbf{E}_{j \rightarrow i}^{\prime}$ is given by Eq. (49), and $\gamma_{j}=\left(1+u_{z j}^{2}\right)^{1 / 2}$ is the relativistic factor. Figure 13 compares Eqs. (58)-(60) to the electrostatic PIC method (Sec. IV), which is a paraxial code. There is agreement both in the transverse and longitudinal dynamics of the electron bunch. The differences between Fig. 13 and Fig. 11 for the PPI results show the deviation from the paraxial approximation.

\section{SM-LWFA injectors using PIC distributions}

In this section we will address the need to use macroparticle distributions containing realistic phase-space correlations as found in SM-LWFA beams, and discuss spacecharge effects using distributions produced by fully electromagnetic PIC codes.

Experiments and simulations using both uniform plasmas and channel guided plasmas have observed substructures in the beam distribution function. Formation of electron bunches with small energy spread followed by a bulk of low energy electrons was observed [33-35] when the plasma length, after injection of background plasma electrons into the plasma wave through the wave breaking process, was on the order of the dephasing length. This length corresponds to the typical distance for a trapped electron to overcome the accelerated region of the wakefield. Typically those electron beams have a high charge on the order of a few nCs and compact dimensions (on the order of the laser pulse size), while the small energy spread sub-bunch has a charge $\sim 500 \mathrm{pC}$ and a length of the order of a plasma wavelength $\lambda_{p}=2 \pi c / \omega_{p}$, where $\omega_{p}=$ $\left(4 \pi n_{0} e^{2} / m_{e}\right)^{1 / 2}$ is the plasma frequency and $n_{0}$ is the plasma density. These beams hence have a very high average density $\bar{n}_{b} \sim 10^{19} \mathrm{~cm}^{-3}$ and, for electrons with $\mathrm{MeV}$ range total energy, space-charge force may play an important role in the beam dynamics while propagating in vacuum.

It has been previously shown (Sec. VIA 1) that the dynamics of beams with small energy spread and divergence are well described by an envelope equation. Furthermore, for moderate space-charge interaction and beams in the space-charge dominated regime, i.e., where the emittance term in the envelope equation is typically small compared to the space-charge term, ellipsoidal symmetric beams with identical initial RMS parameters will exhibit similar growth during propagation. For these beams the effect of initial position space-energy correlation are negligible, whereas for beams with large energy spread, it will strongly affect the changes in time of the distribution function. The full knowledge of the six-dimensional (6D) initial phase-space distribution function is then required for accurate description of the beam dynamics.

Figure 12 shows the beam energy distribution after a propagation distance of $1.5 \mathrm{~mm}$ from an initial constant density electron distribution in real and momentum space. Figure 14 shows a distribution that initially has a linear correlation in the $z-p_{z}$ space after a propagation distance of $9 \mathrm{~mm}$. The linear correlation means that electrons at the front of the bunch have the highest energies and proportionally electrons at the back are the slowest. The input beam parameters were assumed to be a total charge $Q=$ $5 \mathrm{nC}$, a temperature $T_{e}=4 \mathrm{MeV}$, a radius $a=6 \mu \mathrm{m}$, a half-length $a_{3}=10 \mu \mathrm{m}$, and a divergence $x_{0}^{\prime}=2 \mathrm{mrad}$. The space-charge simulations were performed using PPI model (Sec. V). Analysis of the final beam distributions for the two cases of Figs. 12 and 14 confirm the hypothesis that the knowledge of the initial phase-space correlations within the beams are crucial for an accurate description of its time evolution. The beam of Fig. 12 exhibits a peak at 


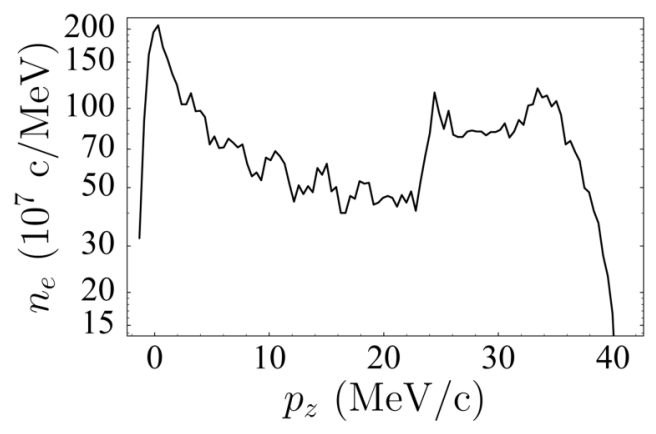

FIG. 14. Final energy distribution for a beam with an initially exponential energy decay law and a longitudinal $z-p_{z}$ linear correlation. Initial parameters: total charge $Q=5 \mathrm{nC}$, temperature $T_{e}=4 \mathrm{MeV}$, radius $a=6 \mu \mathrm{m}$, half-length $a_{3}=10 \mu \mathrm{m}$, and divergence $x_{0}^{\prime}=2 \mathrm{mrad}$.

$p_{z} \simeq 25 \mathrm{MeV} / c$, whereas the example of Fig. 14 shows formation of two peaks. This obviously does not agree with experimental measurements. Note that both cases experience some structural change and, in addition, a substantial gain of energy. This strongly suggests that similar beams parameters may be sensitive to space-charge effects.

In order to get a correct understanding of experimental results, realistic beam distributions may be obtained from self-consistent simulations such as particle-in-cells (PIC) codes $[9,10]$. The mechanism responsible for electron production is the self-modulated laser-wakefieldaccelerator (SM-LWFA) regime [1] in which the laser pulse length $L$ is on the order or greater than the plasma wavelength. PIC simulations using the code VORPAL [36] showed the production of a small energy spread electron bunch in front of a bulk of a high-density electron beam for both an initially uniform plasma and with a preformed plasma channel [33]. The electron bunch lies in the first bucket of the plasma wave and typically has a higher average energy than the rest of the beam, allowing for extraction using a magnetic spectrometer. For moderate laser strength, this can be explained by the combined effect of beam loading and laser pulse evolution that together turn off injection and allows for the formation of a bunch with small energy spread, while further behind the laser pulse, transverse wave breaking may increase the amount of charge injected, resulting in a substantially higher energy spread in this subregion of the beam $(\sim 100 \%)$ and a higher degree of phase mixing $[33,37]$.

Figure 15(a) shows the axial electron beam phase space $z-p_{z}$ for a two-dimensional (2D) slab simulation of a short intense laser pulse propagating through a plasma channel with the following laser-plasma parameters: normalized vector potential $a=e A / m_{e} c^{2}=2.15$, carrier frequency $\lambda_{0}=0.8 \mu \mathrm{m}$, laser duration $\tau=L / c=58 \mathrm{fs}$, spot size at waist $r_{0}=7.3 \mu \mathrm{m}$, background plasma density $n_{0}=1.8 \times 10^{19} \mathrm{~cm}^{-3}, 10$ particles-per-cell, a grid size $\Delta z=90 / 2700=0.03 \mu \mathrm{m}$ axially, $\Delta x=80 / 300=$ $0.26 \mu \mathrm{m}$ transversally, and a parabolic channel of radius $r_{c}=40 \mu \mathrm{m}$. The frame shown corresponds to a laser propagation distance of $\sim 1360 \mu \mathrm{m}$. If the distance exceeds the dephasing length, which in the 1D limit can be defined as $L_{d} \simeq\left(\lambda_{p} / \lambda_{0}\right)^{2} \lambda_{p}$, the beam will strongly evolve to a point where phase mixing smooths the entire bunch. It is crucial to terminate the plasma at a distance on the order of the dephasing length after electron injection into the wakefield to observe experimentally the production of an electron bunch with a small energy spread.

Vacuum transport of the beam shown in Fig. 15 has been performed using the 3D-PPI method. The PIC simulation is currently a $2 \mathrm{D}$ slab, and in order to get a $3 \mathrm{D}$ electron distribution, the output of the code has been assumed cylindrically symmetric. The line-charge density $\lambda_{m}$ associated with a $2 \mathrm{D}$ slab macroparticle is defined as

$$
\lambda_{m}=n_{0} /\left(\Delta x \Delta z N_{p}\right)
$$

where $N_{p}$ is the number of macroparticles per cell. The density is assumed identical between 2D and 3D geometry giving a number of 3D macroparticles $N_{m, i}$ at a radius $r_{i}$ away from the longitudinal axis
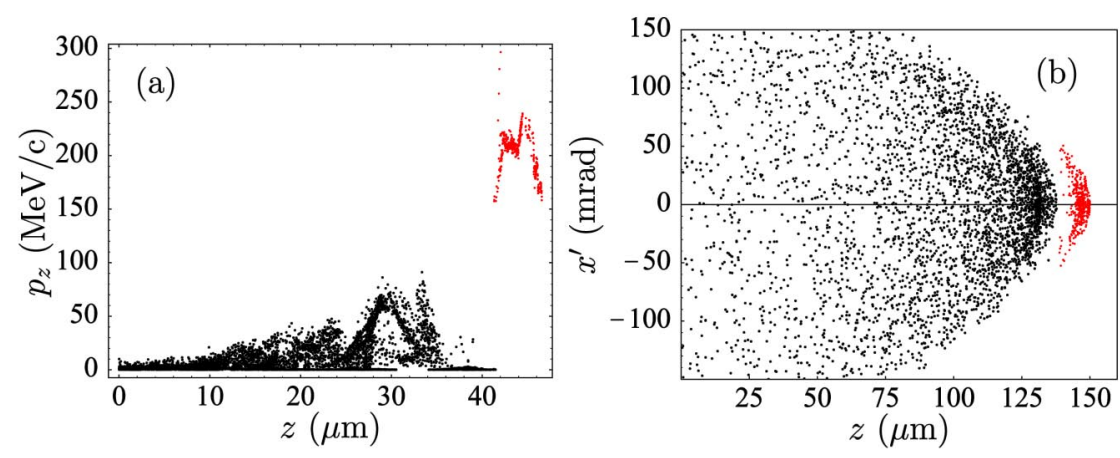

FIG. 15. (Color) $z-p_{z}$ phase-space projection of a 3D electron distribution (a) after a laser propagation distance of $\sim 1.36$ mm inside a preformed plasma channel and (b) $z$ versus beam divergence $x^{\prime}$ after a propagation of $4.8 \mathrm{~mm}$ in vacuum. The laser-plasma parameters were a laser strength $a=2.15$, carrier frequency $\lambda_{0}=0.8 \mu \mathrm{m}$, duration $\tau=L / c=58$ fs, spot size at waist $r_{0}=7.3 \mu \mathrm{m}$, nominal plasma density $n_{0}=1.8 \times 10^{19} \mathrm{~cm}^{-3}$, and channel radius $r_{c}=40 \mu \mathrm{m}$. 


$$
N_{m, i}=2 \pi\left\lfloor r_{i} \lambda_{m}\right\rfloor,
$$

where $r_{i}=\left|y_{i}\right|, y_{i}$ is the transverse coordinate of a 2D slab macroparticle and only half of the slab is considered, i.e., only macroparticles lying in the $y>0$ plane. The 3D electron distribution is populated as follows:

$$
x_{i}^{*}=r_{i} \cos \theta_{i}, \quad y_{i}^{*}=r_{i} \sin \theta_{i}, \quad z_{i}^{*}=z_{i},
$$

where $\left\{x_{i}^{*}, y_{i}^{*}, z_{i}^{*}\right\}$ stand for the Cartesian coordinate of a 3D macroparticle, $\theta_{i}$ is a random number between 0 and $2 \pi$, and $z_{i}$ is the transverse coordinate of a $2 \mathrm{D}$ slab macroparticle. The same transformation has been repeated for the momentum domain $\mathbf{p}_{i}$. The total charge $Q$ in the 3D beam is calculated following

$$
Q=-2 \pi e \sum_{i}^{N_{2 \mathrm{D}}} r_{i} \lambda_{m}
$$

where $N_{2 \mathrm{D}}$ is the number of $2 \mathrm{D}$ slab macroparticles in the $y>0$ plane. For the case of Fig. 15, $N_{p}=10, \Delta x=$ $0.26 \mu \mathrm{m}$, and $\Delta z=0.03 \mu \mathrm{m}$, giving $e \lambda_{m} \simeq 2.5 \mathrm{nC} / \mathrm{m}$. The total charge may vary depending on the selected energy cutoff from the PIC simulation. Taking electrons above $\sim 500 \mathrm{keV}$ within a radius $R=6 \mu \mathrm{m}$ gives a total charge $Q \sim 1.4 \mathrm{nC}$, whereas energies greater than $\sim 100 \mathrm{keV}$ give $Q \sim 3.2 \mathrm{nC}$. The small energy spread beam (red color) contains a charge $Q \sim 25 \mathrm{pC}$. Note that fully 3D PIC simulations shows an enhancement of the amount of charge trapped for a similar resolution in the simulation parameters [38]. Charge in the trapped beam is then found to be closer to experimental observations [33]. In the following, the 3D electron distribution derived from the 2D slab PIC simulation is used as a model for a typical beam produced by laser-plasma interaction. The charge is varied in the space-charge simulation to study its effect on the beam dynamics.

Figure 16(a) shows the initial beam distribution (blue dashed line), final beam distribution (blue solid line) after a propagation distance of $4.8 \mathrm{~mm}$ for a total beam charge of $Q=2.8$ and $Q=8.5 \mathrm{nC}$ (red dashed and solid line), respectively. The latter case clearly exhibits important changes in beam temperature [Fig. 16(c)], which strongly suggests that space-charge effects must be included. Figures 16(b) and 16(d) show a similar behavior. Note that the overall "two-temperature" profile is unchanged, which is in good agreement with experimental observations [22-32]. The latter example assumed a preformed homogeneous plasma and a higher density $n_{0}=$ $4 \times 10^{19} \mathrm{~cm}^{-3}$, which provides a greater ratio of charge trapped for a lower energy gain, consequently increasing the effect of space charge. The resolution used in the PIC simulation is $\Delta x=0.26 \mu \mathrm{m}, \Delta z=0.05 \mu \mathrm{m}$, and $N_{p}=$ 10 providing $e \lambda_{m}=8.3 \mathrm{nC} / \mathrm{m}$. Selecting electrons above $500 \mathrm{keV}$ gives $Q \sim 3.8 \mathrm{nC}$ and $Q \sim 5.1 \mathrm{nC}$ for $E>$ $100 \mathrm{keV}$. The example of Fig. 16(b) is chosen for a charge $Q=5.2 \mathrm{nC}$.

A detailed study of the RMS quantities of the small energy spread electron bunch of Fig. 15 (red color) shows
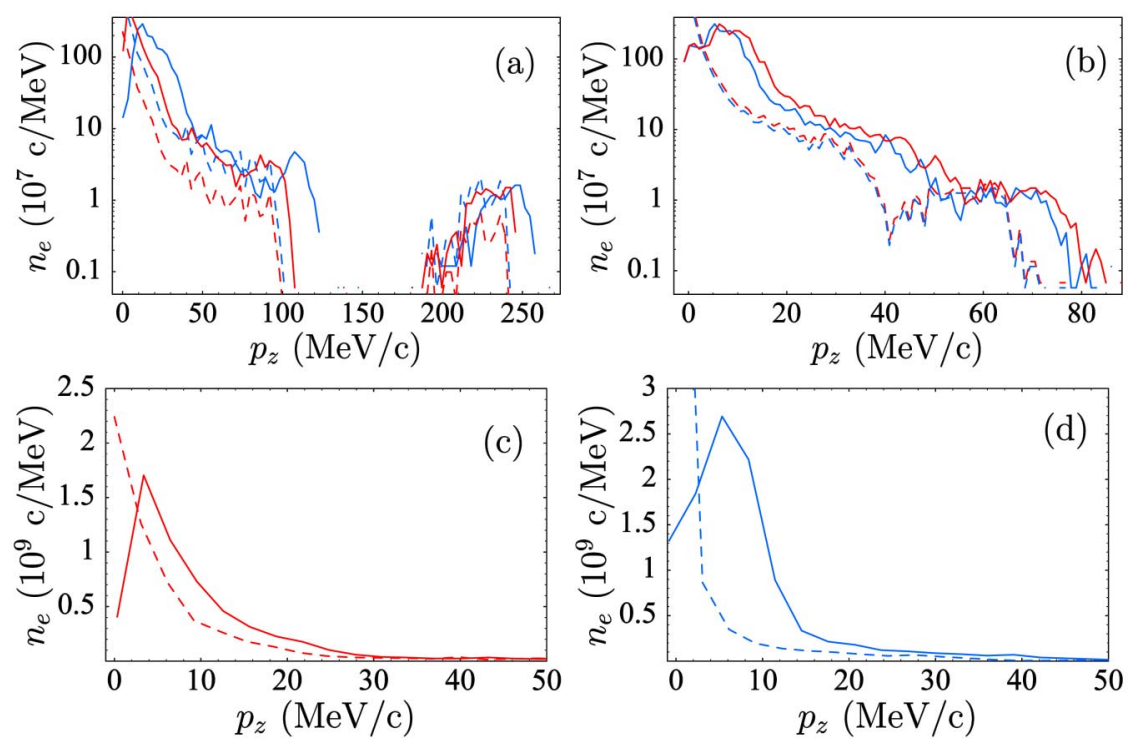

FIG. 16. (Color) (a) Logarithmic plot of the initial beam energy distribution (dashed line) and final (solid line) assuming a total beam charge of $Q=2.8 \mathrm{nC}$ (red) and $Q=8.5 \mathrm{nC}$ (blue) for the parameters of Fig. 15. (b) Same except for a higher density plasma without a preformed channel, assuming a total beam charge of $Q=5.1 \mathrm{nC}$ (blue) and $Q=6 \mathrm{nC}$ (red). A zoom of the low energy region, highlighting the changes in beam temperature induced by space-charge interaction, is shown in (c) for the channeled case [i.e., same as (a)] and in (d) for the single laser pulse experiment [corresponding to the log plot (b)]. The laser-plasma parameters for the latter example were $a=2.2, \lambda_{0}=0.8 \mu \mathrm{m}, \tau=L / c=55 \mathrm{fs}, r_{0}=7.4 \mu \mathrm{m}, n_{0}=4 \times 10^{19} \mathrm{~cm}^{-3}$. 

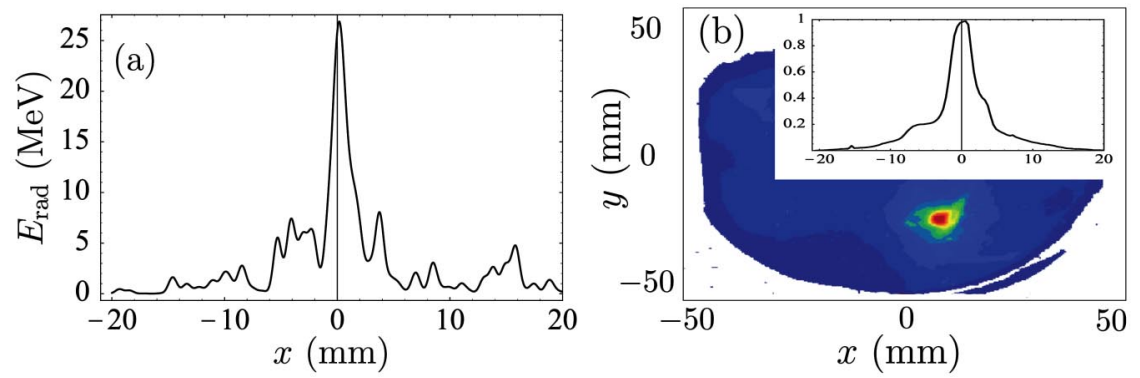

FIG. 17. (Color) Radiated energy on a phosphor screen $70 \mathrm{~cm}$ away from the gas jet, (a) lineout using the electron beam produced by the fully explicit PIC simulation of a channel guided laser pulse [Fig. 15] and (b) radiation from a $10 \times 10 \mathrm{~cm}$ phosphor screen imaged on a CCD camera, which is taken from experimental measurements with similar laser-plasma parameters. The red color highlights the largest energy deposition from the electron beam onto the phosphor and blue the smallest, respectively.

significant energy gain during vacuum propagation while beam quality is maintained, i.e., space-charge interaction with the remaining low energy residual charge of the whole beam induce an energy boost. The total charge in the beam is $3.2 \mathrm{nC}$. At the plasma exit [Fig. 15(a)], the beam has an energy spread $\eta=9.2 \%$, an average energy $T \simeq$ $204.5 \mathrm{MeV}$, a divergence $\sigma_{x^{\prime}} \simeq 16.3 \mathrm{mrad}$, and a bunch length $\sigma_{z} \simeq 1.42 \mu \mathrm{m}$. After $c t=4.8 \mathrm{~mm}$, the energy spread and divergence is found to be a few percent lower, the bunch length is $\sigma_{z} \simeq 2.4 \mu \mathrm{m}$, and the average energy is $T \simeq 209.4 \mathrm{MeV}$ leading to an energy gain of $2.4 \%$ (a total beam charge of $10 \mathrm{nC}$ would induce $\sim 10 \%$ energy gain) and consequently a $2.2 \%$ decrease in trace-space emittance $\epsilon_{x}=\sqrt{\left\langle x^{2}\right\rangle\left\langle x^{\prime 2}\right\rangle-\left\langle x x^{\prime}\right\rangle^{2}}$. Note that, despite the high average energy of the bunch, space charge still has a significant effect. This can be explained in part by the very short dimensions of the whole beam at the vacuum-plasma boundary, on the order of the laser size, giving an average beam number density $\bar{n}_{b} \simeq 3 \times 10^{18} \mathrm{~cm}^{-3}$. In addition, Fig. 15(b) shows the total beam divergence after $4.8 \mathrm{~mm}$ of vacuum propagation, which indicates that the red beam remains ultracompact. This is also similar to experiments, which measure the radiated light emitted by the electron beam passing through a phosphor screen. Figure $17 \mathrm{com}-$ pares the simulated radiated energy from the electron beam produced by the PIC simulation in the channel guided case [Fig. 15] with respect to experimental data from similar laser-plasma parameters. Both cases exhibit a bright emission spot with a full width half maximum (FWHM) of the order of $3 \mathrm{~mm}$.

Similar effects are observed for the unchanneled case $n_{0}=4 \times 10^{19} \mathrm{~cm}^{-3}$ [Fig. 18(a)] but with a significantly higher energy gain. The high energy electron bunch (red color) contains a charge $Q \simeq 55 \mathrm{pC}$ (assuming a total charge of $5.2 \mathrm{nC}$ ). After a $4.8 \mathrm{~mm}$ vacuum propagation distance, the energy spread reduces from $\eta \simeq 12.6 \%$ to $11 \%$, the average energy increases from $T \simeq 55.7 \mathrm{MeV}$ to $65 \mathrm{MeV}$ (energy gain of $16.7 \%$ ) leading to a $12.5 \%$ decrease in trace-space emittance together with a divergence that is lowered by $\sim 9.7 \%$. Besides a more intense spacecharge interaction than in the channeled case, the electron bunch propagates well in vacuum as shown in Fig. 18(b). Note that in both cases some beam compaction and focusing is observed. The latter can be understood as a pinching
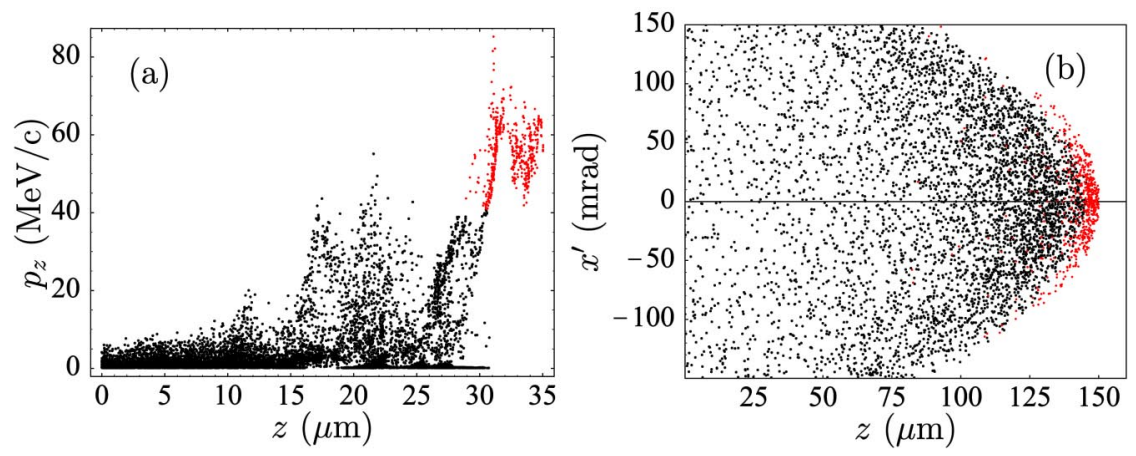

FIG. 18. (Color) $z-p_{z}$ phase-space projection of a 3D electron distribution (a) after a laser propagation distance of $\sim 530 \mu \mathrm{m}$ inside a homogeneous preionized plasma and (b) $z$ versus beam divergence $x^{\prime}$ after a propagation of $5.3 \mathrm{~mm}$ in vacuum. The laser-plasma parameters were a laser strength $a=2.2$, carrier frequency $\lambda_{0}=0.8 \mu \mathrm{m}$, pulse duration $\tau=L / c=55 \mathrm{fs}$, spot size at waist $r_{0}=$ $7.4 \mu \mathrm{m}$, and nominal density $n_{0}=4 \times 10^{19} \mathrm{~cm}^{-3}$. 
effect from the $\theta$-component of the magnetic field generated by the high longitudinal current of the beam as a whole.

\section{COMPARISON BETWEEN PPI AND PIC METHODS}

In this section, we modify the PPI algorithm in order to perform a direct comparison with respect to an electrostatic PIC code in terms of total CPU time and accuracy of the methods. In Sec. VI B 2 we pointed out that beams produced by laser-plasma accelerators in the self-modulated regime will experience significant evolution under spacecharge interaction. Within the paraxial approximation, electron beam evolution can be studied using an electrostatic PIC code or a modified PPI code. An electrostatic PIC code calculates the electric field by a finite-difference scheme from a smoothed density on a grid. This approach requires enough statistics through high numbers of particles per cell (typically greater than 5) and a grid size small enough to resolve internal substructures within the beam (typically $\Delta x_{i} \sim 0.1-1 \mu \mathrm{m}$ ). The electrostatic PIC algorithm used in our simulations has an adaptive grid size, i.e., the grid number is fixed but the grid size is adjusted as the beam evolves. This adaptive technique reduces computing time for the same accuracy goals compared to conventional PIC methods that have a fixed decomposition domain. On the other hand, an advantage of using the PPI technique is that it does not rely on a grid.

In the following, a parameter scan was performed showing parameters needed to solve the beam configuration of Fig. 11 using both an electrostatic PIC code and PPI. For this scan, PPI was modified such that a single beam rest frame was assumed. The fields solver in the PPI code is reduced to

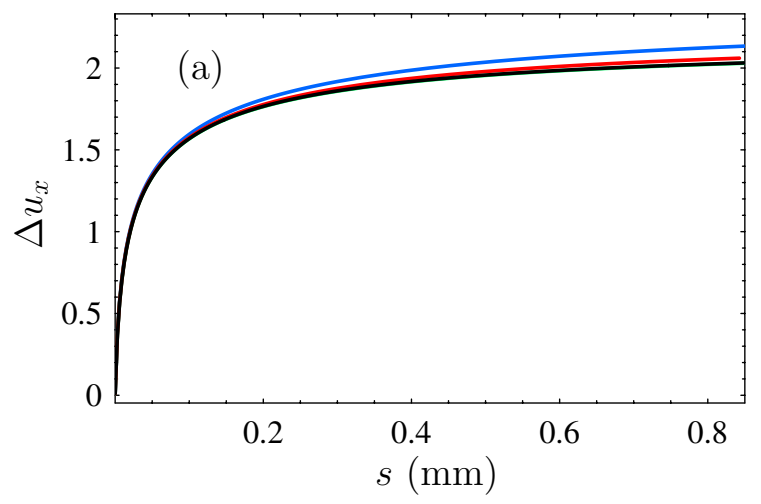

$$
\begin{gathered}
\mathbf{E}_{\perp i}=\bar{\gamma} \sum_{j \neq i} \mathbf{E}_{\perp, j \rightarrow i}^{\prime}, \\
\mathbf{E}_{z i}=\sum_{j \neq i} \mathbf{E}_{z, j \rightarrow i}^{\prime}, \\
c \mathbf{B}_{i}=\bar{\beta}_{z} \mathbf{e}_{z} \times \mathbf{E}_{i},
\end{gathered}
$$

where $\mathbf{E}_{j \rightarrow i}^{\prime}$ is given by Eq. (49), $\bar{\beta}_{z}=\bar{u}_{z} /\left(1+\bar{u}_{z}^{2}\right)^{1 / 2}$ is the average electron normalized velocity, $\bar{\gamma}=\left(1+\bar{u}_{z}^{2}\right)^{1 / 2}$ the average relativistic factor, $\bar{u}_{z}$ the average normalized momentum, and $\mathbf{e}_{z}$ a unit vector. Note that no binning was used for the electrostatic PIC simulation.

Figure 19 shows the comparison between the electrostatic PIC and PPI for the parameters of Fig. 11, i.e., for a bunch charge of $Q=5 \mathrm{nC}$, an exponential profile for the longitudinal momentum with a temperature $k T=4 \mathrm{MeV}$, an initial bunch radius of $a=6 \mu \mathrm{m}$, initial length $a_{3}=$ $10 \mu \mathrm{m}$, and an initial divergence $x_{0}^{\prime}=2 \mathrm{mrad}$. Figures 19(a) and 19(b) plot the transverse and longitudinal RMS momentum spread $\Delta u_{x,(z)}$, respectively, as a function of propagation distance. Figures 19 shows the PPI model (black line) with $N=20032$ macroparticles, $r_{0}=$ $100 \mathrm{~nm}$, and $n_{0}^{-1 / 3}=422 \mathrm{~nm}$, the electrostatic PIC with (i) $N=10.24 \times 10^{6}$ macroparticles and grids $128^{2} \times 512$ (green line), (ii) $N=20.48 \times 10^{6}$ macroparticles and grids $256^{3}$ (red line), and (iii) $N=2.56 \times 10^{6}$ macroparticles and grids $128^{3}$ (blue line). One can see that 128 adaptive grid points transversally are enough for the electrostatic PIC code to reach convergence whereas a longitudinal grid number greater that 256 is necessary. The example shown in green shows very good agreement with PPI. For this particular example, it is found that $\tau=$ $10 \mathrm{~ms} /$ processor/particle is the average PPI's execution time per time step and $\tau=2 \mathrm{~ms} /$ processor/particle for the

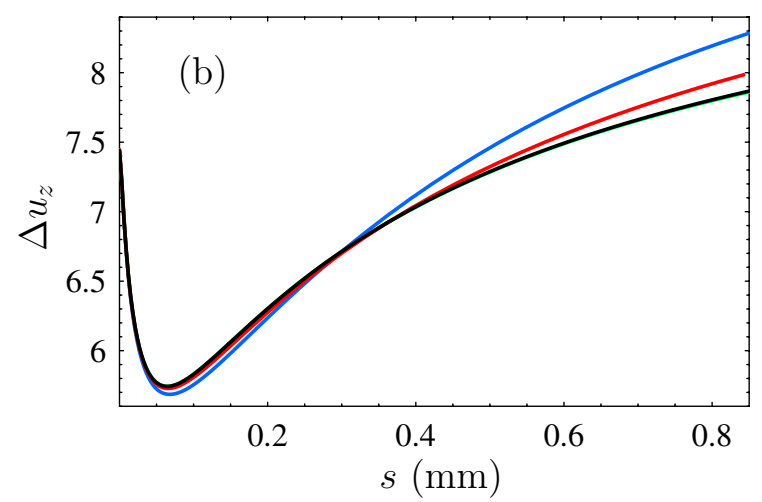

FIG. 19. (Color) Comparison between the reduced 3D-PPI model and the electrostatic PIC for the case of a large energy spread beam with an exponential energy profile. Input parameters: Charge $Q=5 \mathrm{nC}$, temperature $k_{B} T=4 \mathrm{MeV}$, radius $a=6 \mu \mathrm{m}$, half-length $a_{3}=10 \mu \mathrm{m}$, and divergence $x_{\max }^{\prime}=2 \mathrm{mrad}$. In (black) PPI with $N=20032$ macroparticles, cutoff radius $r_{0}=100 \mathrm{~nm}$ and averaged macroparticle distance $n_{0}^{-1 / 3}=422 \mathrm{~nm}$, (green) electrostatic PIC with $N=10.24 \times 10^{6}$ macroparticles and grids $128^{2} \times 512$, (red) electrostatic PIC with $N=20.48 \times 10^{6}$ macroparticles and grids $256^{3}$, and (blue) electrostatic PIC with $N=2.56 \times 10^{6}$ macroparticles and grids $128^{3}$. Evolution of (a) transverse RMS momentum spread $\Delta u_{x}$ and (b) longitudinal RMS momentum spread $\Delta u_{z}$ as a function of propagation distance $s$ is shown. 
electrostatic PIC code. The small number of particles needed for the PPI run made the simulation significantly faster.

\section{GENERALIZATION OF THE PPI MODEL}

The PPI method presented in Sec. V neglected radiation effects and used an approximated form of retardation. The force acting on macroparticle $i$ from macroparticle $j$ was calculated in the laboratory frame using the Lorentztransformed fields from the rest frame of macroparticle $j$, but assumed the event was "instantaneous" in both frames. This assumption implies that within the PPI method spacecharge interaction must remain moderate, yet the PPI method is more general than either the envelope or shell models [see Sec. VA for further details]. One condition that should be satisfied in order to apply the PPI method is the requirement that the potential energy per electron in the beam frame be smaller than the electron rest mass energy. This condition can be written as $\bar{U}^{\prime} / m c^{2} \ll 1$, where $\bar{U}^{\prime}$ is the total potential energy normalized to the number of electrons, which is given approximately by

$$
\bar{U}^{\prime}=U_{0} / N_{e},
$$

where $N_{e}$ is the total number of electrons and $U_{0}$ is identical to Eq. (52) for beams with initially small energy spreads, i.e.,

$$
U_{0} \simeq \int_{V} d^{3} x^{\prime} \frac{\epsilon_{0} \mathbf{E}^{\prime 2}}{2} .
$$

Beams produced by laser-plasma interactions, particularly in the self-modulated regime, have a relatively high charge density giving $\bar{U}^{\prime} / m c^{2} \sim 1$, which implies an upper limit of applicability for the PPI model. As was discussed in Sec. VIB 3, SM-LWFA electron sources have a typical exponential energy density profile, that is the beam is mainly composed of low energy electrons together with a small subset of high energy electrons located at the front of the bunch. The high energy beam dynamics should moderately be influenced by retardation effects because it mainly interacts with the low energy electrons, assuming the latter do not become relativistic during the time of interaction. This assumption is valid as long as the ballistic spreading is significant between the two populations. The opposite is not true but the charge density of the high energy beam is relatively small and has very little effect on the low energy electrons. Last, the relative error introduced by the PPI method for the blowup of the low energy electrons may be crudely estimated using the ideal beam distributions of Sec. VA. The error was found to be on the percent level, which is significantly lower than the overall change of the beam temperature [Fig. 16(c) and 16(d)] discussed in Sec. VIB 3.

For the case $\bar{U}^{\prime} / m c^{2} \gtrsim 1$ retardation and radiation effects may play a role in the overall beam dynamics. A generalization of the PPI model may be computed from the
Lienard-Wiechert fields that are an exact solution of Maxwell's equations (i.e., including both retardation and radiation), that is [39-41]

$$
\begin{aligned}
\mathbf{E}_{i}(\mathbf{r}, t)= & \frac{Q_{m}}{4 \pi \epsilon_{0}}\left\{\left[\frac{\mathbf{n}-\beta_{i}}{\gamma_{i}^{2}\left(1-\mathbf{n} \cdot \beta_{i}\right)^{3} R^{2}}\right]_{\mathrm{RET}}\right. \\
& \left.+\left[\frac{\mathbf{n} \times\left\{\left(\mathbf{n}-\beta_{i}\right) \times \dot{\beta}_{i}\right\}}{c\left(1-\mathbf{n} \cdot \beta_{i}\right)^{3} R}\right]_{\mathrm{RET}}\right\},
\end{aligned}
$$

and

$$
\mathbf{B}_{i}(\mathbf{r}, t)=c^{-1}\left[\mathbf{n} \times \mathbf{E}_{i}\right]_{\mathrm{RET}},
$$

where $Q_{m}$ is the macroparticle charge, $R \mathbf{n}=\mathbf{r}-\mathbf{r}_{i}$ and the subscript "RET" indicates that the quantities in the brackets are to be evaluated at the retarded time $t_{\mathrm{RET}}=$ $t-\left|\mathbf{r}-\mathbf{r}_{i}\left(t_{\mathrm{RET}}\right)\right| / c$. Here, the first and second terms on the right of Eq. (70) represent the self-fields and radiation fields, respectively. For a system of macroparticles interacting via their mutual electromagnetic fields, the equations of motion are

$$
\frac{d \mathbf{p}_{i}}{d t}=Q_{m} \sum_{j \neq i}\left[\mathbf{E}_{j}\left(\mathbf{r}_{i}, t\right)+\mathbf{v}_{i} \times \mathbf{B}_{j}\left(\mathbf{r}_{i}, t\right)\right],
$$

where $\mathbf{E}_{j}$ and $\mathbf{B}_{j}$ are the electromagnetic fields due to the motion of macroparticle $j$ (the condition $j \neq i$ excludes the self-force). The numerical approach is in principle that used to solve retarded scalar-field problems [42]: The kinematic information is stored in arrays and retrieved by interpolation when needed later for the retarded quantities.

One important issue with using macroparticles for problems where radiation is present $\left(\bar{U}^{\prime} / m c^{2} \gtrsim 1\right)$ is the fact that macroparticles yield artificially high levels of radiation. Note that this is not the case concerning the acceleration (i.e., motion) of a macroparticle. Equation (72) indicates that the change in the normalized momentum $d \mathbf{u}_{i} / d t \propto Q_{m} / m_{m}$, where $\mathbf{u}_{i}=\mathbf{p}_{i} / m_{m} c$ is the normalized momentum and $m_{m}$ is the mass of the macroparticle. Hence, provided that the ratio $Q_{m} / m_{m}$ is the same for the macroparticle as it is for a single electron (as is the case), then the motion of the macroparticle is the same as that for a single electron, provided that the fields acting on the particles are the same. This is no longer true concerning radiation. This can be illustrated by considering the Larmor formula for the power radiated $P_{R}$ by a macroparticle that is accelerating along a straight line,

$$
P_{R}=\frac{Q_{m}^{2}}{6 \pi \epsilon_{0} m_{m}^{2} c^{3}}\left(\frac{d p_{i}}{d t}\right)^{2}
$$

Using Eq. (72), the power radiated by a single macroparticle scales as $P_{R} \propto Q_{m}^{4} / m_{m}^{2}$. Hence, assuming $Q_{m} / m_{m}=e / m_{e}$, then the power radiated by a single macroparticle is too large by the factor $\left(Q_{m} / e\right)^{2}=N_{R}^{2}$, where $N_{R}$ is the number of electrons that each macroparticle represents. However, as pointed out by Jackson [43], the radiated power is typically very small compared 
to the change in kinetic energy of the particle. Specifically, the ratio of radiated power to the time rate of change of kinetic energy for a single electron is given by

$$
\frac{P_{R}}{d W_{K} / d t}=\frac{2 r_{e}}{3 \beta m_{e} c^{2}} \frac{d W_{K}}{d x}
$$

where $W_{K}=m_{e} c^{2}\left[\left(1+p^{2} / m_{e}^{2} c^{2}\right)^{1 / 2}-1\right]$ is the kinetic energy of the electron. Hence, for a relativistic electron, the effect of radiation will be small as long as the effective field gradient $d W_{K} / d x$ is small compared to $3 m_{e} c^{2} / 2 r_{e}$, i.e., $\left|d W_{K} / d x\right| \ll 3 \times 10^{14} \mathrm{MeV} / \mathrm{m}$, which is trivially satisfied for a single electron. For a macroparticle, the radiation is $N_{R}^{2}$ times larger, i.e., the right side of Eq. (74) is multiplied by the factor $N_{R}^{2}$. Since $N_{R}$ is very large (typically, $N_{r} \sim 10^{6}$ ), the condition on the effective field gradient required for the effects of radiation to be small is approximately $\left|d W_{K} / d x\right| \ll 300 \mathrm{MeV} / \mathrm{m}$. This can easily be violated. For example, the radial electric field inside the homogeneous spherical charge distribution is given by $E_{r}=e N_{e} r /\left(4 \pi \epsilon_{0} R^{3}\right)$, where $N_{e}$ is the total number of electrons in the bunch and $R$ the bunch radius. For an electron at $r=R$, the field gradient can be written as $e E_{r}=\left(m_{e} c^{2}\right) N_{e} r_{e} / R^{2}$, which gives $e E_{r}=14 \mathrm{GeV} / \mathrm{m}$ for $N_{e}=10^{9}$ and $R=10 \mu \mathrm{m}$. If the radiated fields are to be retained in the PPI method, then the number of macroparticles needs to be increased such that the above condition is satisfied. Alternatively, if the radiated fields do not play a significant role in the physical problem being considered, then the contribution of the radiated fields in Eq. (70) can be neglected, and only the self-fields (with retardation) retained.

\section{CONCLUSION}

Plasma-based accelerators offer the possibility of providing compact, high-gradient electron accelerators and are also capable of producing ultrashort electron bunches in which the longitudinal size is much smaller than the transverse size. Space-charge effects are not of concern while the bunch is in the plasma wave, since the longitu- dinal and transverse fields of the wake are typically much greater than the space-charge fields of the bunch. Space charge may be important, however, when an electron bunch propagates in vacuum with no external fields, as can be the case with a modest energy laser-plasma injector, in which the emerging electron bunch has very compact dimensions, high charge density, relatively low energy (up to few tens of MeVs), and possibly a large energy spread. Conventional space-charge approaches, restricted to small energy spread beams, are not applicable in this case.

In this paper, we explored several analytical and numerical methods for the simulation of beams typical of those produced by laser-plasma injectors. Some properties of these methods are summarized in Table I. These methods include: (i) The coupled envelope equations, which assume that the beam shape remains ellipsoidal with a small divergence (paraxial approximation) and small energy spread (typically on the order of a few \%). This model can be applied to most sources produced by optical injection such as colliding pulse (CPI). (ii) A novel technique that uses ellipsoidal symmetric beam distribution functions and allows the treatment of large energy spreads. It assumes the paraxial approximation and allows an extension of the envelope model to beams with large energy spreads. This method has the advantage of being computationally fast compared to standard techniques such as particle-incell (PIC). (iii) A modified electrostatic PIC method, allowing for the binning in momentum of the beams with large energy spread. (iv) A 3D point-to-point interaction (PPI) method, which neglects the radiated fields and treats retardation in an approximate manner. The PPI and PIC codes have been compared and applied to the simulations of high density, compact electron beams where the paraxial approximation is violated. This is relevant to the electron sources produced by the self-modulated laser wakefield accelerator (SM-LWFA). The number density for these beams is typically $n_{e} \sim 10^{19} \mathrm{~cm}^{-3}$, which results in significant space-charge blowup while propagating in vacuum. The distribution in phase-space evolves rapidly for those dense beams and its final shape is strongly affected by the initial correlations.

TABLE I. Some properties of four methods (coupled envelope equations, ellipsoidal shells, modified electrostatic particle-in-cell, and point-to-point interaction) used to model electron bunch evolution.

\begin{tabular}{lcccc}
\hline \hline & Envelope & Shell & PIC & PPI \\
\hline & & & & \\
Energy spread & Narrow & Broad & Broad & Broad \\
Momentum bins & One & Multiple & Multiple & None \\
Paraxial & Yes & Yes & Yes & No \\
Symmetry & Ellipsoidal & Ellipsoidal & Arbitrary & Arbitrary \\
Macroparticles & No & Yes & Yes & Yes \\
Impact parameter & No & No & No & Yes $^{\mathrm{a}}$ \\
\hline \hline
\end{tabular}

${ }^{\mathrm{a}}$ The PPI model is also limited by the effects of radiation and retardation (see text). 
Studying in detail the SM-LWFA regime, it was found that position-momentum correlations have an important effect on the evolution of the electron distribution for large energy spread beams. Electromagnetic PIC simulations of the SM-LWFA typically yield highly correlated beams with strong substructure. Such beams are typically composed of a train of beamlets (bunched at the plasma period), with the highest energies in the first beamlet and lower energies in the trailing beamlet. This is typical provided the beam is extracted after a propagation distance within the plasma on the order of the dephasing length [33]. It was found that space charge plays a major role and can induce electron self-acceleration with a global change of beam energy spectrum while keeping an overall "twotemperature" profile at the location of the detector. This is consistent with experimental observations [22-32]. Furthermore, it has been shown that while propagating in vacuum the higher energy electrons within the beam can gain significant energy (up to $16 \%$ for the examples considered).

\section{ACKNOWLEDGMENTS}

The authors acknowledge useful discussions with C. B. Schroeder, B. A Shadwick, C. Geddes, R. Ryne, and J. Faure. This work was performed under the auspices the U.S. Department of Energy, Office of High Energy Physics, under Contract No. E-AC-02-05CH11231, and by the U.S. Department of Energy SciDAC project, "Advanced Computing for 21st Century Accelerator Science and Technology," which is supported by the Office of High Energy Physics and the Office of Advanced Scientific Computing Research. This research used resources of the National Energy Research Scientific Computing Center.

\section{APPENDIX A: ELECTROSTATIC FIELD OF A HETEROGENEOUS ELLIPSOID}

In this Appendix, a summary on potential theory applied to a charge distribution with ellipsoidal symmetry is provided. An excellent derivation is given in the book Ellipsoidal Figures of Equilibrium from Chandrasekhar [12], which will not be detailed here. For simplicity, we will assume that the charge distribution is at rest and that the ellipsoid is located at the center of the frame of reference, i.e., $\bar{x}_{i}=0$. For a solid homogeneous ellipsoid with density distribution $\rho\left(r^{2}\right)$, the potential [12] is

$$
\phi(x, y, z)=\frac{a_{1} a_{2} a_{3}}{4 \epsilon_{0}} \int_{0}^{\infty} \frac{d u}{\Delta(u)} \int_{r^{2}(u)}^{1} d r^{2} \rho\left(r^{2}\right),
$$

where

$$
r^{2}=\sum_{i} \frac{x_{i}^{2}}{a_{i}^{2}}
$$

$$
r^{2}(u)=\sum_{i} \frac{x_{i}^{2}}{a_{i}^{2}+u}
$$

and

$$
\Delta(u)=\sqrt{\left(a_{1}^{2}+u\right)\left(a_{2}^{2}+u\right)\left(a_{3}^{2}+u\right)} .
$$

The general form for the electric field is

$$
E_{i}=-\frac{\partial \phi}{\partial x_{i}}=-\int_{0}^{\infty} \frac{\partial f(x, y, z, u)}{\partial x_{i}} d u,
$$

with

$$
f(x, y, z, u)=\frac{1}{4 \epsilon_{0}} \frac{a_{1} a_{2} a_{3}}{\Delta(u)} \int_{r^{2}(u)}^{1} d r^{2} \rho\left(r^{2}\right),
$$

and

$$
\frac{\partial f(x, y, z, u)}{\partial x_{i}}=-\frac{1}{4 \epsilon_{0}} \frac{a_{1} a_{2} a_{3}}{\Delta(u)} \rho\left[r^{2}(u)\right] \frac{\partial r^{2}(u)}{\partial x_{i}},
$$

i.e.,

$$
E_{i}=\frac{a_{1} a_{2} a_{3}}{2 \epsilon_{0}} x_{i} \int_{0}^{\infty} \frac{d u}{\left(a_{i}^{2}+u\right) \Delta(u)} \rho\left[r^{2}(u)\right] .
$$

For the special case where the density profile has the form

$$
\rho\left(r^{2}\right)= \begin{cases}\rho_{0}, & \text { for } r^{2} \leq 1 \\ 0, & \text { otherwise }\end{cases}
$$

we get inside the ellipsoid,

$$
E_{i}=\frac{\rho_{0}}{2 \epsilon_{0}} a_{1} a_{2} a_{3} x_{i} A_{i}(0, \infty)
$$

and for a point outside the ellipsoid, Eq. (A3) gives $r^{2}(\lambda)=1$ where $\lambda>0$ and the integral may be rewritten as

$$
E_{i}=\frac{\rho_{0}}{2 \epsilon_{0}} a_{1} a_{2} a_{3} x_{i} A_{i}(\lambda, \infty)
$$

where

$$
A_{i}(\lambda, \infty)=\int_{\lambda}^{\infty} \frac{d u}{\left(a_{i}^{2}+u\right) \Delta(u)}
$$

For a round beam $a_{1}=a_{2}=a$, we have

$$
A_{i}(\lambda, \infty)=\int_{\lambda}^{\infty} \frac{d u}{\left(a_{i}^{2}+u\right)\left(a^{2}+u\right) \sqrt{a_{3}^{2}+u}},
$$

where 


$$
2 \lambda(x, y, z)=x^{2}+y^{2}+z^{2}-a^{2}-a_{3}^{2}+\sqrt{\left(x^{2}+y^{2}+z^{2}-a^{2}-a_{3}^{2}\right)^{2}+4\left[a_{3}^{2}\left(x^{2}+y^{2}\right)+a^{2}\left(z^{2}-a_{3}^{2}\right)\right]} .
$$

These results are used in Sec. III for the derivation of the envelope equations and, in the next Appendix, for the calculation of the fields of an homogeneous ellipsoidal shell.

\section{APPENDIX B: ELECTROSTATIC FIELD OF A HOMOGENEOUS ELLIPSOIDAL SHELL}

The density profile of an homogeneous ellipsoidal shell can be defined as

$$
\rho\left(r^{2}\right)= \begin{cases}\rho_{0}, & \text { for } m_{0}^{2} \leq r^{2} \leq m_{1}^{2} \\ 0, & \text { otherwise }\end{cases}
$$

Using a transform of variables $r \rightarrow u$, as in the previous Appendix, the limits of integration for a shell become $u=$ $\left(u_{0}, u_{1}\right)$, where $u_{0}$ is the positive root of the equation $r^{2}\left(u_{0}\right)=m_{0}^{2}$ and $u_{1}$ the positive root of $r^{2}\left(u_{1}\right)=m_{1}^{2}$. Three regions can then be defined depending of the point of observation.
Region A: In the interior of a shell (inside both ellipsoids), the field is zero,

$$
E_{i}=0
$$

Region B: Inside of a shell (between the ellipsoid boundaries),

$$
E_{i}=\frac{\rho_{0}}{2 \epsilon_{0}} x_{i} A_{i}\left(0, u_{0}\right)
$$

Region C: Exterior of a shell (outside both ellipsoid),

$$
E_{i}=\frac{\rho_{0}}{2 \epsilon_{0}} x_{i} A_{i}\left(u_{1}, u_{0}\right)
$$

For the special case of a round beam $\left(a_{1}=a_{2}=a\right)$, we have

$$
A_{i}\left(u_{1}, u_{0}\right)=\int_{u_{1}}^{u_{0}} \frac{d u}{\left(a_{i}^{2}+u\right)\left(a^{2}+u\right) \sqrt{a_{3}^{2}+u}},
$$

where $u_{i}=\lambda_{i} / m_{i}^{2}$ and

$$
2 \lambda_{i}=x^{2}+y^{2}+z^{2}-m_{i}^{2}\left(a^{2}+a_{3}^{2}\right)+\sqrt{\left[x^{2}+y^{2}+z^{2}-m_{i}^{2}\left(a^{2}+a_{3}^{2}\right)\right]^{2}+4 m_{i}^{2}\left[a_{3}^{2}\left(x^{2}+y^{2}\right)+a^{2}\left(z^{2}-m_{i}^{2} a_{3}^{2}\right)\right]} .
$$

The space-charge coefficients are

$$
A=A_{1}=A_{2}=g_{0}\left(u_{0}\right)-g_{0}\left(u_{1}\right), A_{3}=h_{0}\left(u_{0}\right)-h_{0}\left(u_{1}\right) \text {, }
$$

with

$$
\begin{aligned}
g_{0}(u)= & \frac{1}{a^{2}-a_{3}^{2}}\left\{\frac{\sqrt{a_{3}^{2}+u}}{a^{2}+u}\right. \\
& \left.+\frac{\tan ^{-1}\left[\sqrt{a_{3}^{2}+u} / \sqrt{a^{2}-a_{3}^{2}}\right]}{\sqrt{a^{2}-a_{3}^{2}}}\right\},
\end{aligned}
$$

and

$$
\begin{aligned}
h_{0}(u)= & \frac{2}{a_{3}^{2}-a^{2}}\left\{\frac{1}{\sqrt{a_{3}^{2}+u}}\right. \\
& \left.+\frac{\tan ^{-1}\left[\sqrt{a_{3}^{2}+u} / \sqrt{a^{2}-a_{3}^{2}}\right]}{\sqrt{a^{2}-a_{3}^{2}}}\right\} .
\end{aligned}
$$

These equations give a full description of the electrostatic fields acting on an ellipsoidal shell. Section II generalized these results to moving shells and presented the outline of a new approach that was applied to beams with large energy spreads through binning of the electron distribution in momentum space, providing a computationally fast and efficient method to calculate space-charge effects for beams in the paraxial limit, that is, when transverse blowup is moderate $\left(\left|\beta_{\perp}\right| \ll \beta_{z}\right)$.

\section{APPENDIX C: HAMILTONIAN FORMULATION OF THE RMS ENVELOPE EQUATIONS}

This Appendix presents a general derivation of the RMS envelope equations using a Hamiltonian approach. This derivation does not depend on a detailed knowledge of the electron distribution function. Appendix D will further specialize to ellipsoidal symmetric bunches and Appendix E to the special case of finite cylinder of charge.

\section{Single particle equation of motion}

This section closely follows the work of Ryne et al. [17]. Starting from the usual definition linking the Lagrangian to the Hamiltonian,

$$
L=p \dot{q}-H,
$$

and using the definition of the action,

$$
S=\int(p \dot{q}-H) d t
$$

one can derive an expression for the Lagrangian in a coordinate system where $z$ is the independent variable, i.e., 


$$
\begin{gathered}
d S=(p \dot{q}-H) d t, \\
=\left(p_{x} x^{\prime}+p_{y} y^{\prime}+p_{z}-H t^{\prime}\right) d z,
\end{gathered}
$$

with $p_{i}=\gamma m v_{i}+q A_{i}$. Defining $p_{t}=-H$, we get

$$
\begin{gathered}
S=\int\left(p_{x} x^{\prime}+p_{y} y^{\prime}+p_{t} t^{\prime}+p_{z}\right) d z \\
=\int \hat{L} d z
\end{gathered}
$$

where $\hat{L}=p_{x} x^{\prime}+p_{y} y^{\prime}+p_{t} t^{\prime}-K$ and $K=-p_{z}$. From the expression of the Hamiltonian in terms of the independent variable $t$,

$$
\begin{gathered}
H\left(x, p_{x}, y, p_{y}, z, p_{z} ; t\right)=\sqrt{m^{2} c^{4}+c^{2}\left[\left(p_{x}-q A_{x}\right)^{2}+\left(p_{y}-q A_{y}\right)^{2}+\left(p_{z}-q A_{z}\right)^{2}\right]}+q \Phi=-p_{t}, \\
\mathbf{B}(x, y, z, t)=\nabla \times \mathbf{A}(x, y, z, t), \\
\mathbf{E}(x, y, z, t)=-\nabla \Phi(x, y, z, t)-\frac{\partial \mathbf{A}(x, y, z, t)}{\partial t} .
\end{gathered}
$$

It is readily found a similar expression where $z$ is now the independent variable, i.e.,

$$
\begin{gathered}
K\left(x, p_{x}, y, p_{y}, t, p_{t} ; z\right)=-p_{z}, \\
=-\sqrt{\left(p_{t}+q \Phi\right)^{2} / c^{2}-m^{2} c^{2}-\left(p_{x}-q A_{x}\right)^{2}-\left(p_{y}-q A_{y}\right)^{2}}-q A_{z} .
\end{gathered}
$$

For the case of space charge, i.e., no external applied fields, $A_{x}^{\text {self }}=A_{y}^{\text {self }}=0$ and $A_{z}^{\text {self }}=v_{0} \Phi^{\text {self }} / c^{2}$,

$$
K=-\sqrt{\left(p_{t}+q \Phi^{\text {self }}\right)^{2} / c^{2}-m^{2} c^{2}-p_{x}^{2}-p_{y}^{2}}-q A_{z}^{\text {self }},
$$

and using variational principles,

$$
\begin{gathered}
p_{t}=-\frac{\partial K}{\partial t}, \\
t=\frac{\partial K}{\partial p_{t}}, \\
\ldots
\end{gathered}
$$

we can deduce the equations of motion in $z$ frame [17].

\section{Reference orbit}

In a general manner the reference orbit is identical to the trajectory of a reference particle that evolves in the fields of the beam transport system (quadrupole magnets, rf accelerating cavities, ...) from some initial conditions carefully chosen in order to simplify the form of the overall equations that describe the evolution of any particles in the system. Let $\left(t_{0}, p_{t 0}\right)$ denote the trajectory of the reference particle (along with $x=p_{x}=y=p_{y}=0$ to simplify). Using the above Hamiltonian, it follows that the equations of motion for the reference particle are given by

$$
t_{0}^{\prime}=\frac{-p_{t 0}}{\sqrt{p_{t 0}^{2}-m^{2} c^{4}}}=\frac{1}{v_{0}},
$$

$$
p_{t 0}^{\prime}=-\frac{\partial K}{\partial t_{0}}
$$

where primes stand for $d / d z$ and $K$ is the general Hamiltonian [Eq. (C11)] containing the external fields as well as the self-fields. $\Phi$ has been neglected in (C15) because for a beam evolving in vacuum under space-charge blowup alone the reference particle can simply be chosen as to be located at the center of the bunch (the center of force) such that $p_{t 0}^{\prime}=0$, i.e., $p_{t 0}=-H=-\gamma_{0} m c^{2}$ and $t_{0}(z)=z / v_{0}$, where $v_{0}$ is the average beam velocity. This assumption is valid as long as the beam energy spread $\eta=$ $\Delta E / E_{0}$ remains small.

\section{Equations in the frame comoving with the reference orbit}

Next define variables that are deviations from the reference orbit,

$$
\begin{aligned}
& X=x, \quad P_{x}=p_{x}, \quad Y=y, \quad P_{y}=p_{y}, \\
& T=t-t_{0}(z), \quad P_{t}=p_{t}-p_{t 0}(z) .
\end{aligned}
$$

The Hamiltonian governing this variables is obtained from the generating function

$$
F_{2}(q, P)=x P_{x}+y P_{y}+\left[t-t_{0}(z)\right]\left[P_{t}+p_{t 0}(z)\right],
$$


according to

and

$$
\begin{gathered}
Q=\frac{\partial F_{2}}{\partial P}, \\
p=\frac{\partial F_{2}}{\partial q}
\end{gathered}
$$

$$
K^{\text {new }}=K+\frac{\partial F_{2}}{\partial z}
$$

$$
K\left(X, P_{x}, Y, P_{y}, T, P_{t} ; z\right)=-\sqrt{\left[\left(P_{t}+p_{t 0}\right)+q \Phi^{\text {self }}\right]^{2} / c^{2}-m^{2} c^{2}-P_{x}^{2}-P_{y}^{2}}-q A_{z}^{\text {self }}+T \frac{d p_{t 0}}{d z}-\left(P_{t}+p_{t 0}\right) \frac{d t_{0}}{d z},
$$

where

$$
\begin{aligned}
& \mathbf{A}^{\text {self }}=\mathbf{A}^{\text {self }}\left(X, Y, z, T+t_{0}\right), \\
& \Phi^{\text {self }}=\Phi^{\text {self }}\left(X, Y, z, T+t_{0}\right) .
\end{aligned}
$$

Next we will substitute the potentials into the Hamiltonian and drop terms of order 3 and higher in $\left(X, P_{x}, P_{y}, Y, T, P_{t}\right)$. Also, since $\Phi^{\text {self }}$ is of order 2 and higher, we will keep $\Phi^{\text {self }}$ but drop $\left(\Phi^{\text {self }}\right)^{2}$ and higher powers. It follows that the Hamiltonian is given by

$$
K=-p_{0} \sqrt{1-\frac{2\left(P_{t}+q \Phi^{\text {self }}\right)}{p_{0} v_{0}}+\frac{P_{t}^{2}}{p_{0}^{2} c^{2}}-\frac{P_{x}^{2}}{p_{0}^{2}}-\frac{P_{y}^{2}}{p_{0}^{2}}}-\frac{v_{0}}{c^{2}} q \Phi^{\text {self }}-\frac{P_{t}}{v_{0}}+\gamma_{0} m c^{2} / v_{0},
$$

where $p_{0}=\gamma_{0} m v_{0}$ has been used along with $A_{Z}^{\text {self }}=$ $v_{0} \Phi^{\text {self }} / c^{2}$. To second order in $\left(X, P_{x}, Y, P_{y}, T, P_{t}\right)$, Eq. (C24) becomes

$$
K \simeq \frac{m^{2} P_{t}^{2}}{2 p_{0}^{3}}+\frac{P_{x}^{2}+P_{y}^{2}}{2 p_{0}}-\frac{q \Phi^{\text {self }}}{\gamma_{0}^{2} v_{0}}-p_{0}\left(1-\beta_{0}^{-2}\right) .
$$

Note that $\beta_{0}^{-2}=1+p_{0}^{-2} m^{2} c^{2}$. The last term in the Hamiltonian is not a function of the canonical variables and consequently does not contribute to the dynamics of the electrons. Therefore, it will not be considered in the calculation. The new expression for the Hamiltonian is

$$
K\left(X, P_{x}, Y, P_{y}, T, P_{t} ; z\right)=\frac{m^{2} P_{t}^{2}}{2 p_{0}^{3}}+\frac{P_{x}^{2}+P_{y}^{2}}{2 p_{0}}-\frac{q \Phi^{\text {self }}}{\gamma_{0}^{2} v_{0}} .
$$

It follows from Hamilton's equations that the equations of motion are given by

$$
\begin{gathered}
X^{\prime}=\frac{P_{x}}{p_{0}}, \\
P_{x}^{\prime}=-\frac{q}{\gamma_{0}^{2} v_{0}} \frac{\partial}{\partial X} \Phi^{\text {self }}, \\
Y^{\prime}=\frac{P_{y}}{p_{0}}, \\
P_{y}^{\prime}=-\frac{q}{\gamma_{0}^{2} v_{0}} \frac{\partial}{\partial Y} \Phi^{\text {self }},
\end{gathered}
$$

$$
\begin{gathered}
T^{\prime}=\frac{m^{2} P_{t}}{p_{0}^{3}} \\
P_{t}^{\prime}=-\frac{q}{\gamma_{0}^{2} v_{0}} \frac{\partial}{\partial T} \Phi^{\text {self }} .
\end{gathered}
$$

These can be combined in pairs to yield the following three equations:

$$
\begin{aligned}
X^{\prime \prime}+\frac{q}{\gamma_{0}^{2} p_{0} v_{0}} \frac{\partial}{\partial X} \Phi^{\text {self }} & =0 \\
Y^{\prime \prime}+\frac{q}{\gamma_{0}^{2} p_{0} v_{0}} \frac{\partial}{\partial Y} \Phi^{\text {self }} & =0 \\
T^{\prime \prime}+\frac{q}{\gamma_{0}^{4} p_{0} v_{0}^{3}} \frac{\partial}{\partial T} \Phi^{\text {self }} & =0 .
\end{aligned}
$$

Recall that $p_{0}(z)$ was assumed to be constant, i.e., $p_{0}^{\prime}=0$.

\section{RMS envelope equations}

Evolution equations for the RMS moments of the beam can be obtained using standard techniques. Consider, for example, the calculation of $\left\langle X^{2}\right\rangle$. First, let

$$
\sigma_{x}=\sqrt{\left\langle X^{2}\right\rangle}
$$

giving

$$
\sigma_{x}^{\prime}=\frac{\left\langle X X^{\prime}\right\rangle}{\sigma_{x}}=\frac{1}{p_{0}} \frac{\left\langle X P_{x}\right\rangle}{\sigma_{x}}
$$

and 


$$
\sigma_{x}^{\prime \prime}=\frac{\left\langle X P_{x}^{\prime}\right\rangle}{p_{0} \sigma_{x}}+\frac{\epsilon_{x}^{2}}{p_{0}^{2} \sigma_{x}^{3}},
$$

where the normalized emittance was introduced as

$$
\epsilon_{x}=\sqrt{\left\langle X^{2}\right\rangle\left\langle P_{x}^{2}\right\rangle-\left\langle X P_{x}\right\rangle^{2}} .
$$

The RMS envelope in the $x$ direction is then

$$
\sigma_{x}^{\prime \prime}+\frac{q}{p_{0} v_{0} \gamma_{0}^{2}} \frac{1}{\sigma_{x}}\left\langle X \frac{\partial}{\partial X} \Phi^{\text {self }}\right\rangle-\frac{\epsilon_{x}^{2}}{p_{0}^{2} \sigma_{x}^{3}}=0 .
$$

Similarly, the equations for the other second moments are given by

$$
\begin{gathered}
\sigma_{y}^{\prime \prime}+\frac{q}{p_{0} v_{0} \gamma_{0}^{2}} \frac{1}{\sigma_{y}}\left\langle Y \frac{\partial}{\partial Y} \Phi^{\text {self }}\right\rangle-\frac{\epsilon_{y}^{2}}{p_{0}^{2} \sigma_{y}^{3}}=0, \\
\sigma_{t}^{\prime \prime}+\frac{q}{p_{0} v_{0}^{3} \gamma_{0}^{4}} \frac{1}{\sigma_{t}}\left\langle T \frac{\partial}{\partial T} \Phi^{\text {self }}\right\rangle-\frac{\epsilon_{t}^{2}}{p_{0}^{2} \gamma_{0}^{4} v_{0}^{4} \sigma_{t}^{3}}=0,
\end{gathered}
$$

and

$$
\begin{gathered}
\epsilon_{y}=\sqrt{\left\langle Y^{2}\right\rangle\left\langle P_{y}^{2}\right\rangle-\left\langle Y P_{y}\right\rangle^{2}}, \\
\epsilon_{t}=\sqrt{\left\langle T^{2}\right\rangle\left\langle P_{t}^{2}\right\rangle-\left\langle T P_{t}\right\rangle^{2}} .
\end{gathered}
$$

Furthermore, defining $Z$ as the distance between the particle and the reference particle

$$
Z(z)=v_{0} T(z),
$$

where $T=t-t_{0}$ is the time difference between the two particles at position $z$, then multiplying Eq. (C40) by $\boldsymbol{v}_{0}$ gives an expression for the longitudinal envelope equation in term of the $Z$ coordinate,

$$
\sigma_{z}^{\prime \prime}+\frac{q}{p_{0} v_{0} \gamma_{0}^{4}} \frac{1}{\sigma_{z}}\left\langle Z \frac{\partial}{\partial Z} \Phi^{\text {self }}\right\rangle-\frac{\epsilon_{z}^{2}}{\gamma_{0}^{4} \sigma_{z}^{3}}=0,
$$

along with

$$
\epsilon_{z}=\sqrt{\left\langle Z^{2}\right\rangle\left\langle\delta^{2}\right\rangle-\langle Z \delta\rangle^{2}}
$$

where $\delta=P_{t} /\left(p_{0} v_{0}\right)$. Note that it is easy to show that, if $\Phi^{\text {self }}$ is a quadratic function of $X, Y$, and $Z$ (or equivalently, if we simply neglect higher order terms), then the normalized RMS emittances are constants.

\section{APPENDIX D: COUPLED ENVELOPE EQUATIONS IN ELLIPSOIDAL GEOMETRY}

For the space-charge force calculation, the approximation $Z=v_{0} T \simeq z-z_{0}(t)$ is strictly valid only if the beam energy spread is small. Recall that the space-charge forces can only be calculated in a frame where $t$ is the independent variable. Next we will consider the calculation of the terms $\left\langle X \partial \Phi^{\text {self }} / \partial X\right\rangle,\left\langle Y \partial \Phi^{\text {self }} / \partial Y\right\rangle$, and $\left\langle Z \partial \Phi^{\text {self }} / \partial Z\right\rangle$ in the envelope equations. Assume that the charge density of a single bunch is given by

$$
\rho(X, Y, Z)=\frac{Q}{4 \pi a_{1} a_{2} a_{3}} h\left(\frac{X^{2}}{a_{1}^{2}}+\frac{Y^{2}}{a_{2}^{2}}+\frac{Z^{2}}{a_{3}^{2}}\right) .
$$

The charge per bunch is equal to $Q$. By making the substitutions

$$
\begin{gathered}
X=a_{1} r \sin \theta \cos \phi, \\
Y=a_{2} r \sin \theta \sin \phi, \\
Z=a_{3} r \cos \theta,
\end{gathered}
$$

it follows that

$$
\begin{aligned}
Q & =\iiint_{V} \rho d^{3} \mathbf{X} \\
& =\frac{Q}{4 \pi} \int_{0}^{\infty} r^{2} h\left(r^{2}\right) d r \int_{0}^{\pi} \sin \theta d \theta \int_{0}^{2 \pi} d \phi,
\end{aligned}
$$

i.e., $h\left(r^{2}\right)$ is normalized according to

$$
\int_{0}^{\infty} r^{2} h\left(r^{2}\right) d r=1
$$

It is also straightforward to show that the second order moments are related to $a_{1}, a_{2}$, and $a_{3}$ as shown below:

$$
\begin{aligned}
& \left\langle X^{2}\right\rangle=\frac{a_{1}^{2}}{3} \int_{0}^{\infty} r^{4} h\left(r^{2}\right) d r, \\
& \left\langle Y^{2}\right\rangle=\frac{a_{2}^{2}}{3} \int_{0}^{\infty} r^{4} h\left(r^{2}\right) d r, \\
& \left\langle Z^{2}\right\rangle=\frac{a_{3}^{2}}{3} \int_{0}^{\infty} r^{4} h\left(r^{2}\right) d r .
\end{aligned}
$$

As an example let us assume a constant ellipsoid. The boundary is defined by the ellipsoidal coordinate

$$
r_{\max }^{2}=\frac{X^{2}}{a_{1}^{2}}+\frac{Y^{2}}{a_{2}^{2}}+\frac{Z^{2}}{a_{1}^{3}}=1,
$$

we get

$$
h\left(r^{2}\right)=3
$$

and

$$
\begin{aligned}
& \left\langle X^{2}\right\rangle=a_{1}^{2} / 5, \\
& \left\langle Y^{2}\right\rangle=a_{2}^{2} / 5, \\
& \left\langle Z^{2}\right\rangle=a_{3}^{2} / 5 .
\end{aligned}
$$

The scalar potential in the laboratory frame [Eq. (A1)] is given by 


$$
\Phi^{\text {self }}(X, Y, Z)=\frac{\gamma_{0} Q}{16 \pi \epsilon_{0}} \int_{0}^{\infty} \frac{d s}{\Delta(s)} \int_{m^{2}(s)}^{1} h\left(m^{2}\right) d m^{2},
$$

where

$$
\Delta(s)=\sqrt{\left(a_{1}^{2}+s\right)\left(a_{2}^{2}+s\right)\left(\gamma_{0}^{2} a_{3}^{2}+s\right)},
$$

and

$$
m^{2}(s)=\frac{X^{2}}{a_{1}^{2}+s}+\frac{Y^{2}}{a_{2}^{2}+s}+\frac{\gamma_{0}^{2} Z^{2}}{\gamma_{0}^{2} a_{3}^{2}+s} .
$$

The calculation of $\mathbf{E}$ [Eq. (A8)] is straightforward:

$$
\begin{aligned}
& E_{x}=-\frac{\partial}{\partial X} \Phi^{\text {self }}=\frac{\gamma_{0} Q}{8 \pi \epsilon_{0}} X \int_{0}^{\infty} \frac{h\left[m^{2}(s)\right] d s}{\left(a_{1}^{2}+s\right) \Delta(s)}, \\
& E_{y}=-\frac{\partial}{\partial Y} \Phi^{\text {self }}=\frac{\gamma_{0} Q}{8 \pi \epsilon_{0}} Y \int_{0}^{\infty} \frac{h\left[m^{2}(s)\right] d s}{\left(a_{2}^{2}+s\right) \Delta(s)},
\end{aligned}
$$

and

$$
\begin{aligned}
E_{z} & =-\frac{\partial}{\partial z} \Phi^{\text {self }}-\frac{\partial}{\partial t} A_{z}^{\text {self }}=-\frac{1}{\gamma_{0}^{2}} \frac{\partial}{\partial Z} \Phi^{\text {self }} \\
& =\frac{\gamma_{0} Q}{8 \pi \epsilon_{0}} Z \int_{0}^{\infty} \frac{h\left[m^{2}(s)\right] d s}{\left(\gamma_{0}^{2} a_{3}^{2}+s\right) \Delta(s)} .
\end{aligned}
$$

The term $\left\langle X \partial \Phi^{\text {self }} / \partial X\right\rangle$ is therefore

$$
\begin{aligned}
\left\langle X \frac{\partial}{\partial X} \Phi^{\text {self }}\right\rangle= & -\frac{\gamma_{0} Q}{8 \pi \epsilon_{0}} \\
& \times \iiint_{V} \frac{\rho\left[m^{2}(0)\right]}{Q} d^{3} \mathbf{X} \int_{0}^{\infty} \frac{X^{2} h\left[m^{2}(s)\right] d s}{\left(a_{1}^{2}+s\right) \Delta(s)},
\end{aligned}
$$

which suggests the change of variables

$$
X=\sqrt{a_{1}^{2}+} s r \sin \theta \cos \phi
$$

$$
Y=\sqrt{a_{2}^{2}+s} r \sin \theta \sin \phi
$$

$$
\gamma_{0} Z=\sqrt{\gamma_{0}^{2} a_{3}^{2}+s} r \cos \theta,
$$

and deducing from the Jacobian determinant the relationship between $d^{3} \mathbf{X}$ and $d r d \theta d \phi$, that is

$$
\gamma_{0} d^{3} \mathbf{X}=\Delta(s) r^{2} d r \sin \theta d \theta d \phi
$$

we have

$$
\begin{aligned}
\left\langle X \frac{\partial}{\partial X} \Phi^{\text {self }}\right\rangle= & \frac{-Q}{32 \pi^{2} \epsilon_{0} a_{1} a_{2} a_{3}} \int_{0}^{\infty} r^{2} h\left(r^{2}\right) d r \int_{0}^{\pi} d \theta \int_{0}^{2 \pi} d \phi \\
& \times \int_{0}^{\infty} r^{2} \sin ^{3} \theta \cos ^{2} \phi h\left[r^{2}+s r^{2}\left(\frac{\sin ^{2} \theta \cos ^{2} \phi}{a_{1}^{2}}+\frac{\sin ^{2} \theta \sin ^{2} \phi}{a_{2}^{2}}+\frac{\cos ^{2} \theta}{\gamma_{0}^{2} a_{3}^{2}}\right)\right] d s
\end{aligned}
$$

After performing the change of variables

$$
\mu^{2}(s)=r^{2}+s r^{2}\left(\frac{\sin ^{2} \theta \cos ^{2} \phi}{a_{1}^{2}}+\frac{\sin ^{2} \theta \sin ^{2} \phi}{a_{2}^{2}}+\frac{\cos ^{2} \theta}{\gamma_{0}^{2} a_{3}^{2}}\right)
$$

one gets

$$
\begin{aligned}
\left\langle X \frac{\partial}{\partial X} \Phi^{\text {self }}\right\rangle= & \frac{-Q}{16 \pi^{2} \epsilon_{0} a_{1} a_{2} a_{3}} \\
& \times \int_{0}^{\infty} r^{2} h\left(r^{2}\right) d r \int_{r}^{\infty} \mu h\left(\mu^{2}\right) d \mu \int_{0}^{\pi} d \theta \int_{0}^{2 \pi} \frac{\sin ^{3} \theta \cos ^{2} \phi d \phi}{\sin ^{2} \theta \cos ^{2} \phi / a_{1}^{2}+\sin ^{2} \theta \sin ^{2} \phi / a_{2}^{2}+\cos ^{2} \theta /\left(\gamma_{0}^{2} a_{3}^{2}\right)},
\end{aligned}
$$

and further making use of the integral identity [12]

$$
I=\int_{0}^{\pi} \sin \theta d \theta \int_{0}^{2 \pi} d \phi \frac{\sin ^{2} \theta \cos ^{2} \phi}{\sin ^{2} \theta \cos ^{2} \phi / a_{1}^{2}+\sin ^{2} \theta \sin ^{2} \phi / a_{2}^{2}+\cos ^{2} \theta /\left(\gamma_{0}^{2} a_{3}^{2}\right)}=2 \pi a_{1}^{3} a_{2} \gamma_{0} a_{3} A_{1}\left(a_{1}, a_{2}, \gamma_{0} a_{3}\right),
$$

where

$$
A_{i}\left(u_{1}, u_{2}, u_{3}\right)=\int_{0}^{\infty} \frac{d s}{\left(u_{i}^{2}+s\right) \Delta(s)},
$$

and,

$$
\Delta(s)=\sqrt{\left(u_{1}^{2}+s\right)\left(u_{2}^{2}+s\right)\left(u_{3}^{2}+s\right)},
$$

we obtain a simplified expression for the average spacecharge term in the envelope equation, i.e., 


$$
\begin{aligned}
\left\langle X \frac{\partial}{\partial X} \Phi^{\text {self }}\right\rangle= & \frac{-\gamma_{0} Q}{8 \pi \epsilon_{0}} a_{1}^{2} A_{1}\left(a_{1}, a_{2}, \gamma_{0} a_{3}\right) \\
& \times \int_{0}^{\infty} r^{2} h\left(r^{2}\right) d r \int_{r}^{\infty} \mu h\left(\mu^{2}\right) d \mu
\end{aligned}
$$

Now introduce the notation [16]

$$
\begin{aligned}
\lambda_{3}= & \frac{1}{3 \sqrt{3}}\left[\int_{0}^{\infty} r^{2} h\left(r^{2}\right) d r \int_{r}^{\infty} \mu h\left(\mu^{2}\right) d \mu\right] \\
& \times\left\{\int_{0}^{\infty} r^{4} h\left(r^{2}\right) d r\right\}^{1 / 2},
\end{aligned}
$$

and noting that

$$
\begin{aligned}
a_{1}^{2} A_{1}\left(\sigma_{x}, \sigma_{y}, \gamma_{0} \sigma_{z}\right)= & \frac{1}{3 \sqrt{3}}\left\{\int_{0}^{\infty} r^{4} h\left(r^{2}\right) d r\right\}^{1 / 2} 3 \sigma_{x}^{2} \\
& \times \int_{0}^{\infty} \frac{d s}{\left(\sigma_{x}^{2}+s\right) \Delta(s)},
\end{aligned}
$$

we get

$$
\left\langle X \frac{\partial}{\partial X} \Phi^{\text {self }}\right\rangle=\frac{-3 \gamma_{0} Q \lambda_{3}}{8 \pi \epsilon_{0}} \sigma_{x}^{2} A_{1}\left(\sigma_{x}, \sigma_{y}, \gamma_{0} \sigma_{z}\right) .
$$

Sacherer et al. [16] also showed that the value of $\lambda_{3}$ is insensitive to the functional form of $h$ and is approximately equal to $1 /(5 \sqrt{5})$. Putting this all together, we obtain the RMS envelope equation

$$
\sigma_{x}^{\prime \prime}-\frac{3}{2} \frac{N r_{e}}{\gamma_{0}^{2} \beta_{0}^{2}} \lambda_{3} \sigma_{x} A_{1}\left(\sigma_{x}, \sigma_{y}, \gamma_{0} \sigma_{z}\right)-\frac{\tilde{\epsilon}_{x}^{2}}{\sigma_{x}^{3}}=0,
$$

where the number of electrons $N$ and the classical electron radius $r_{e}=e^{2} /\left(4 \pi \epsilon_{0} m c^{2}\right)$ has been introduced. Note also that the quantities $A_{i}$ and $\lambda_{3}$ can be viewed as geometrical factors related to space-charge effects. Similarly it follows that

$$
\begin{aligned}
& \left\langle Y \frac{\partial}{\partial Y} \Phi^{\text {self }}\right\rangle=\frac{-3 \gamma_{0} Q \lambda_{3}}{8 \pi \epsilon_{0}} \sigma_{y}^{2} A_{2}\left(\sigma_{x}, \sigma_{y}, \gamma_{0} \sigma_{z}\right), \\
& \left\langle Z \frac{\partial}{\partial Z} \Phi^{\text {self }}\right\rangle=\frac{-3 \gamma_{0}^{3} Q \lambda_{3}}{8 \pi \epsilon_{0}} \sigma_{z}^{2} A_{3}\left(\sigma_{x}, \sigma_{y}, \gamma_{0} \sigma_{z}\right),
\end{aligned}
$$

and

$$
\begin{aligned}
& \sigma_{y}^{\prime \prime}-\frac{3}{2} \frac{N r_{e}}{\gamma_{0}^{2} \beta_{0}^{2}} \lambda_{3} \sigma_{y} A_{2}\left(\sigma_{x}, \sigma_{y}, \gamma_{0} \sigma_{z}\right)-\frac{\tilde{\epsilon}_{y}^{2}}{\sigma_{y}^{3}}=0, \\
& \sigma_{z}^{\prime \prime}-\frac{3}{2} \frac{N r_{e}}{\gamma_{0}^{2} \beta_{0}^{2}} \lambda_{3} \sigma_{z} A_{3}\left(\sigma_{x}, \sigma_{y}, \gamma_{0} \sigma_{z}\right)-\frac{\tilde{\epsilon}_{z}^{2}}{\sigma_{z}^{3}}=0,
\end{aligned}
$$

where $\tilde{\boldsymbol{\epsilon}}_{x}=\boldsymbol{\epsilon}_{x} / p_{0}, \tilde{\boldsymbol{\epsilon}}_{y}=\epsilon_{y} / p_{0}$, and $\tilde{\boldsymbol{\epsilon}}_{z}=\boldsymbol{\epsilon}_{z} / \gamma_{0}^{2}$ are the RMS trace-space emittances defined as

$$
\begin{aligned}
& \tilde{\boldsymbol{\epsilon}}_{x}=\sqrt{\left\langle X^{2}\right\rangle\left\langle X^{\prime 2}\right\rangle-\left\langle X X^{\prime}\right\rangle^{2}}, \\
& \tilde{\boldsymbol{\epsilon}}_{y}=\sqrt{\left\langle Y^{2}\right\rangle\left\langle Y^{\prime 2}\right\rangle-\left\langle Y Y^{\prime}\right\rangle^{2}},
\end{aligned}
$$

$$
\tilde{\boldsymbol{\epsilon}}_{z}=\sqrt{\left\langle Z^{2}\right\rangle\left\langle Z^{\prime 2}\right\rangle-\left\langle Z Z^{\prime}\right\rangle^{2}}
$$

\section{APPENDIX E: FIELDS ACTING ON A FINITE CYLINDER OF CHARGE IN THE LONG BEAM LIMIT}

A derivation of the electromagnetic fields acting on a finite cylinder of charge, in the long beam limit approximation, is summarized in this Appendix. The long beam limit corresponds to the case $a /\left(\gamma a_{3}\right) \ll 1$, where $a$ is the transverse radius, $\gamma$ the beam relativistic factor, and $a_{3}$ the beam half-length, respectively. Appendix E 1 introduces the general expression for the potential of a finite cylinder in integral form. The latter result will be applied in Appendices E 2 and E 3 to the calculation of the fields and, in Appendix E 4 (also Sec. III B), to the derivation of an envelope equation, for the specific case of a beam with a longitudinal quadratic density profile and uniform radial profile.

\section{General results}

The potential of a static charge distribution $\tilde{\boldsymbol{\rho}}(\tilde{\boldsymbol{r}})$ in cylindrical coordinates is

$$
\begin{aligned}
\tilde{V}(\tilde{r}, \tilde{\theta}, \tilde{z})= & k \int_{-\infty}^{\infty} d \tilde{z}^{\prime} \int_{0}^{\infty} d \tilde{r}^{\prime} \\
& \times \int_{0}^{2 \pi} d \tilde{\theta}^{\prime} \frac{\tilde{\rho}\left(\tilde{\boldsymbol{r}}^{\prime}\right)}{\sqrt{\left(\tilde{z}-\tilde{z}^{\prime}\right)^{2}+\left|\tilde{\boldsymbol{r}}-\tilde{\boldsymbol{r}}^{\prime}\right|^{2}}},
\end{aligned}
$$

where

$$
k=\frac{1}{4 \pi \epsilon_{0}} .
$$

This is the potential in a system at rest with respect to the charge distribution (rest frame potentials, fields, and coordinates are designated with tildes). If the charge distribution is moving along the $z$ axis with velocity $\beta c$, then to transform to the laboratory we use

$$
\begin{gathered}
\rho=\tilde{\rho} \gamma \\
\boldsymbol{r}=\tilde{\boldsymbol{r}}
\end{gathered}
$$$$
z-z^{\prime}=\left(\tilde{z}-\tilde{z}^{\prime}\right) / \gamma
$$

so that the potential in the rest frame, in terms of laboratory coordinates, is

$$
\begin{aligned}
\tilde{V}(r, \theta, z)= & \frac{k}{\gamma} \int_{-\infty}^{\infty} d z^{\prime} \int_{0}^{\infty} d r^{\prime} \\
& \times \int_{0}^{2 \pi} d \theta^{\prime} \frac{\rho\left(\boldsymbol{r}^{\prime}\right)}{\sqrt{\left(z-z^{\prime}\right)^{2}+\left|\boldsymbol{r}-\boldsymbol{r}^{\prime}\right|^{2} / \gamma^{2}}}
\end{aligned}
$$

Let us suppose that the charge density $\rho(\boldsymbol{r})$ is cylindrically symmetric and separable in the product form 


$$
\rho(\boldsymbol{r})=\sigma(r) \lambda(z)
$$

with the normalization

$$
\iiint_{V} \rho(\boldsymbol{r}) d^{3} \boldsymbol{r}=e N
$$

giving

$$
\int_{-\infty}^{\infty} \lambda(z) d z=e N
$$

and

$$
2 \pi \int_{0}^{\infty} \sigma(r) r d r=1,
$$

where $N$ is the number of electrons. Then, with $\boldsymbol{u}=\boldsymbol{r} / \gamma$, and letting $z^{\prime} \rightarrow z^{\prime}-z$, we have

$$
\begin{aligned}
\tilde{V}(r, \theta, z)= & k \gamma \int_{0}^{\infty} u^{\prime} \sigma\left(u^{\prime} \gamma\right) d u^{\prime} \int_{0}^{2 \pi} d \theta^{\prime} \\
& \times \int_{-\infty}^{\infty} d z^{\prime} \frac{\lambda\left(z^{\prime}+z\right)}{\sqrt{z^{\prime 2}+\left|\boldsymbol{u}-\boldsymbol{u}^{\prime}\right|^{2}}} .
\end{aligned}
$$

We will consider only charge densities $\lambda(z)$ that vanish for $|z|>a_{3}$. In this case, we have

$$
\begin{aligned}
\tilde{V}(r, \theta, z)= & k \gamma \int_{0}^{\infty} u^{\prime} \sigma\left(u^{\prime} \gamma\right) d u^{\prime} \int_{0}^{2 \pi} d \theta^{\prime} \\
& \times \int_{-a_{3}-z}^{a_{3}-z} d z^{\prime} \frac{\lambda\left(z^{\prime}+z\right)}{\sqrt{z^{\prime 2}+\left|\overline{\boldsymbol{u}}-\overline{\boldsymbol{u}}^{\prime}\right|^{2}}}
\end{aligned}
$$

\section{Quadratic density profile}

For a parabolic charge density profile in the $z$ direction,

$$
\lambda(z)=\frac{3 e N}{4 a_{3}}\left(1-\frac{z^{2}}{a_{3}^{2}}\right)
$$

using

$$
\begin{aligned}
\int_{-a_{3}-z}^{a_{3}-z} d z^{\prime} \frac{\lambda\left(z^{\prime}+z\right)}{\sqrt{z^{\prime 2}+\left|\mathbf{u}-\mathbf{u}^{\prime}\right|^{2}}}= & \frac{3 e N}{8 a_{3}^{3}}\left\{-\left(a_{3}+3 z\right) \sqrt{\left(a_{3}-z\right)^{2}+\left|\mathbf{u}-\mathbf{u}^{\prime}\right|^{2}}-\left(a_{3}-3 z\right) \sqrt{\left(a_{3}+z\right)^{2}+\left|\mathbf{u}-\mathbf{u}^{\prime}\right|^{2}}\right. \\
& \left.+\left(2 a_{3}^{2}-2 z^{2}+\left|\mathbf{u}-\mathbf{u}^{\prime}\right|^{2}\right) \ln \left[\frac{a_{3}-z+\sqrt{\left(a_{3}-z\right)^{2}+\mid \mathbf{u}-\overline{\left.\mathbf{u}^{\prime}\right|^{2}}}}{-a_{3}-z+\sqrt{\left(a_{3}+z\right)^{2}+\mid \mathbf{u}-\overline{\left.\mathbf{u}^{\prime}\right|^{2}}}}\right]\right\}
\end{aligned}
$$

we now let $\delta=\mathbf{u} / a_{3}, \delta^{\prime}=\mathbf{u}^{\prime} / a_{3}$, and we further make the approximation $|\delta|=r /\left(\gamma a_{3}\right) \ll 1$. Expanding in powers of $\left|\delta-\delta^{\prime}\right|$ and keeping only the leading order terms gives

$$
\begin{aligned}
- & \left(a_{3}+3 z\right) \sqrt{\left(a_{3}-z\right)^{2}+\left|\mathbf{u}-\mathbf{u}^{\prime}\right|^{2}} \\
& -\left(a_{3}-3 z\right) \sqrt{\left(a_{3}+z\right)^{2}+\left|\mathbf{u}-\mathbf{u}^{\prime}\right|^{2}} \simeq-2 a_{3}^{2}+6 z^{2},
\end{aligned}
$$

and

$$
\frac{a_{3}-z+\sqrt{\left(a_{3}-z\right)^{2}+\mid \mathbf{u}-\overline{\left.\mathbf{u}^{\prime}\right|^{2}}}}{-a_{3}-z+\sqrt{\left(a_{3}+z\right)^{2}+\left|\mathbf{u}-\overline{\mathbf{u}}^{\prime}\right|^{2}}} \simeq \frac{4\left(a_{3}^{2}-z^{2}\right)}{\left|\mathbf{u}-\mathbf{u}^{\prime}\right|^{2}} .
$$

Note that such an approximation is only valid for $|z|<a_{3}$. The fields at the edge of the cylinder are not properly defined. We get

$$
\begin{aligned}
\tilde{V}(r, \theta, z) \simeq & \frac{3 k e N \gamma}{4 a_{3}^{3}} \int_{0}^{\infty} u^{\prime} \sigma\left(u^{\prime} \gamma\right) d u^{\prime} \int_{0}^{2 \pi} d \theta^{\prime}\left\{-a_{3}^{2}+3 z^{2}\right. \\
& \left.+\left(a_{3}^{2}-z^{2}\right)\left[\ln 4\left(a_{3}^{2}-z^{2}\right)-\ln \left|\mathbf{u}-\mathbf{u}^{\prime}\right|^{2}\right]\right\}
\end{aligned}
$$

i.e.,

$$
\begin{aligned}
\tilde{V}(r, \theta, z) \simeq & \frac{3 k \pi e N \gamma}{2 a_{3}^{3}} \int_{0}^{\infty} u^{\prime} \sigma\left(u^{\prime} \gamma\right) d u^{\prime}\left[-a_{3}^{2}+3 z^{2}+\left(a_{3}^{2}\right.\right. \\
& \left.\left.-z^{2}\right) \ln 4\left(a_{3}^{2}-z^{2}\right)\right]-\frac{3 k e N \gamma}{4 a_{3}^{3}}\left(a_{3}^{2}-z^{2}\right) \\
& \times \int_{0}^{\infty} u^{\prime} \sigma\left(u^{\prime} \gamma\right) d u^{\prime} \int_{0}^{2 \pi} d \theta^{\prime} \ln \left|\mathbf{u}-\mathbf{u}^{\prime}\right|^{2} .
\end{aligned}
$$

There is only one term that is difficult to integrate in this expression:

$$
\int_{0}^{\infty} u^{\prime} \sigma\left(u^{\prime} \gamma\right) d u^{\prime} \int_{0}^{2 \pi} d \theta^{\prime} \ln \left|\mathbf{u}-\mathbf{u}^{\prime}\right|^{2}
$$

in which

$$
\left|\mathbf{u}-\mathbf{u}^{\prime}\right|^{2}=u^{2}+u^{\prime 2}-2 u u^{\prime} \cos \left(\theta-\theta^{\prime}\right) .
$$

To do the $\theta^{\prime}$ integration, we observe that due to cylindrical symmetry the result must be independent of $\theta$ and consequently we can choose the location $\theta=0$. Using the identity (for $b<1$ )

$$
\int_{0}^{2 \pi} \ln (1-b \cos x) d x=2 \pi \ln \frac{1+\sqrt{1-b^{2}}}{2}
$$

and writing 


$$
\left|\mathbf{u}-\mathbf{u}^{\prime}\right|^{2}=\left(u^{2}+u^{\prime 2}\right)\left(1-\frac{2 u u^{\prime}}{u^{2}+u^{\prime 2}} \cos \theta^{\prime}\right),
$$

we see that

$$
b=\frac{2 u u^{\prime}}{u^{2}+u^{\prime 2}}
$$

and

$$
\frac{1+\sqrt{1-b^{2}}}{2}=\frac{u^{2}+u^{\prime 2}+\left|u^{2}-u^{\prime 2}\right|}{2\left(u^{2}+u^{\prime 2}\right)}
$$

that is,

$$
\begin{aligned}
\int_{0}^{2 \pi} d \theta \ln \left|\mathbf{u}-\mathbf{u}^{\prime}\right|^{2}= & \int_{0}^{2 \pi} d \theta\left[\ln \left(u^{2}+u^{\prime 2}\right)\right. \\
& \left.+\ln \left(1-\frac{2 u u^{\prime}}{u^{2}+u^{\prime 2}} \cos \theta^{\prime}\right)\right] \\
= & 2 \pi \ln \left(\frac{u^{2}+u^{\prime 2}+\left|u^{2}-u^{\prime 2}\right|}{2}\right) .
\end{aligned}
$$

Thus if $u^{\prime}<u$,

$$
\int_{0}^{2 \pi} d \theta \ln \left|\mathbf{u}-\mathbf{u}^{\prime}\right|^{2}=2 \pi \ln u^{2}
$$

while if $u<u^{\prime}$,

$$
\int_{0}^{2 \pi} d \theta \ln \left|\mathbf{u}-\mathbf{u}^{\prime}\right|^{2}=2 \pi \ln u^{\prime 2}
$$

and Eq. (E19) becomes

$$
\begin{aligned}
& \int_{0}^{\infty} u^{\prime} \sigma\left(u^{\prime} \gamma\right) d u^{\prime} \int_{0}^{2 \pi} d \theta^{\prime} \ln \left|\mathbf{u}-\mathbf{u}^{\prime}\right|^{2} \\
& =2 \pi\left(\ln u^{2} \int_{0}^{u} u^{\prime} \sigma\left(u^{\prime} \gamma\right) d u^{\prime}+\int_{u}^{\infty} u^{\prime} \ln u^{\prime 2} \sigma\left(u^{\prime} \gamma\right) d u^{\prime}\right)
\end{aligned}
$$

providing a new expression for the potential

$$
\begin{aligned}
\tilde{V}(r, \theta, z) \simeq & \frac{3 k \pi e N \gamma}{2 a_{3}^{3}}\left\{\int _ { 0 } ^ { \infty } u ^ { \prime } \sigma ( u ^ { \prime } \gamma ) d u ^ { \prime } \left[-a_{3}^{2}+3 z^{2}\right.\right. \\
& \left.+\left(a_{3}^{2}-z^{2}\right) \ln 4\left(a_{3}^{2}-z^{2}\right)\right] \\
& -\left(a_{3}^{2}-z^{2}\right)\left(\ln u^{2} \int_{0}^{u} u^{\prime} \sigma\left(u^{\prime} \gamma\right) d u^{\prime}\right. \\
& \left.\left.+\int_{u}^{\infty} u^{\prime} \ln u^{\prime 2} \sigma\left(u^{\prime} \gamma\right) d u^{\prime}\right)\right\}
\end{aligned}
$$

\section{Uniform beam}

\section{a. Fields inside the beam}

For a uniform beam of radius $a$, we have, for $r<a$,

$$
\sigma(r)=\frac{1}{\pi a^{2}}
$$

Then for $u<a / \gamma$,

$$
\ln u^{2} \int_{0}^{u} u^{\prime} \sigma\left(u^{\prime} \gamma\right) d u^{\prime}=\left(2 \pi a^{2}\right)^{-1} u^{2} \ln u^{2},
$$

and

$$
\begin{aligned}
\int_{u}^{\infty} u^{\prime} \ln u^{\prime 2} \sigma\left(u^{\prime} \gamma\right) d u= & \left(2 \pi a^{2} \gamma^{2}\right)^{-1}\left[u^{2}\left(1-\ln \frac{u^{2}}{\gamma^{2}}\right)\right. \\
& \left.-a^{2}\left(1-\ln \frac{a^{2}}{\gamma^{2}}\right)\right]
\end{aligned}
$$

giving

$$
\begin{aligned}
\tilde{V}(r, \theta, z) \simeq & \frac{3 k e N}{4 \gamma a_{3}^{3}}\left\{-a_{3}^{2}+3 z^{2}+\left(a_{3}^{2}-z^{2}\right)\left[\ln 4\left(a_{3}^{2}-z^{2}\right)\right.\right. \\
& \left.\left.-\ln \frac{a^{2}}{\gamma^{2}}-\frac{u^{2} \gamma^{2}}{a^{2}}+1\right]\right\}
\end{aligned}
$$

The longitudinal electric field is

$$
\begin{aligned}
\tilde{E}_{z} & =-\frac{\partial \tilde{V}}{\partial \tilde{z}}=-\frac{1}{\gamma} \frac{\partial \tilde{V}}{\partial z} \\
& \simeq-\frac{3 k e N z}{\gamma^{2} a_{3}^{3}}\left[1-\ln \left(\frac{2 \gamma \sqrt{a_{3}^{2}-z^{2}}}{a}\right)-\frac{1}{2}\left(1-\frac{r^{2}}{a^{2}}\right)\right],
\end{aligned}
$$

i.e.,

$$
\tilde{E}_{z} \simeq-\frac{2 k \lambda^{\prime}(z)}{\gamma^{2}}\left[\ln \left(\frac{2 \gamma \sqrt{a_{3}^{2}-z^{2}}}{a}\right)-\frac{1}{2}\left(1+\frac{r^{2}}{a^{2}}\right)\right]
$$

The transverse electric field is

$$
\begin{aligned}
\tilde{E}_{r} & =-\frac{\partial \tilde{V}}{\partial \tilde{r}}=-\frac{1}{\gamma} \frac{\partial \tilde{V}}{\partial u} \\
& \simeq \frac{3 k e N}{2 \gamma a^{2} a_{3}^{3}} r\left(a_{3}^{2}-z^{2}\right) \simeq \frac{2 k \lambda(z) r}{\gamma a^{2}}
\end{aligned}
$$

Figure 20 plots the ratio $E_{z}^{*} / E_{z}$ on axis, for a quadratic density profile [Eq. (E13)]. $E_{z}^{*}$ casts for the longitudinal electric field obtained from direct integration of Eq. (E1). One can see an excellent agreement with the approximate expression Eq. (E35) up to the limit $z \simeq a_{3}$, with

$$
\lim _{z \rightarrow a_{3}} E_{z}(0, \theta, z)=-\infty
$$

The fields at the edge of the cylinder are not properly defined because of the assumptions made in Eq. (E15) and (E16) that allow for an analytical solution to be found. $E_{z}$ is strictly valid for $|z|<a_{3}, a /\left(\gamma a_{3}\right) \ll 1$ (long beam) and $r /\left(\gamma a_{3}\right) \ll 1$. 


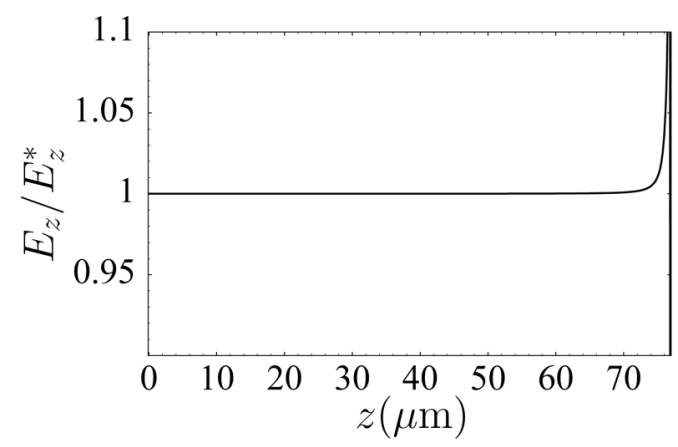

FIG. 20. $E_{z}^{*}(0, \theta, z) / E_{z}(0, \theta, z)$ using a parabolic density profile [Eq. (E13)] and for $a_{0}=19 \mu \mathrm{m}, a_{3}=77 \mu \mathrm{m}$, and $\gamma_{0}=13.7$. $E_{z}^{*}$ corresponds to the longitudinal electric field obtained from direct integration of Eq. (E1). Recall that $z=0$ is the center of the bunch.

\section{b. Fields outside the beam}

For $u>a / \gamma$

$$
\int_{u}^{\infty} u^{\prime} \ln u^{\prime 2} \sigma\left(u^{\prime} \gamma\right) d u^{\prime}=0,
$$

and

$$
\ln u^{2} \int_{0}^{u} u^{\prime} \sigma\left(u^{\prime} \gamma\right) d u^{\prime}=\left(2 \pi \gamma^{2}\right)^{-1} \ln u^{2},
$$

giving

$$
\begin{aligned}
\tilde{V}(r, \theta, z) \simeq & \frac{3 k e N}{4 \gamma a_{3}^{3}}\left\{-a_{3}^{2}+3 z^{2}+\left(a_{3}^{2}-z^{2}\right)\right. \\
& \left.\times\left[\ln 4\left(a_{3}^{2}-z^{2}\right)-\ln u^{2}\right]\right\} .
\end{aligned}
$$

The longitudinal electric field is

$$
\tilde{E}_{z}=-\frac{1}{\gamma} \frac{\partial \tilde{V}}{\partial z} \simeq-\frac{2 k \lambda^{\prime}(z)}{\gamma^{2}}\left[\ln \left(\frac{2 \gamma \sqrt{a_{3}^{2}-z^{2}}}{r}\right)-1\right] .
$$

The transverse electric field is

$$
\tilde{E}_{r}=-\frac{\partial \tilde{V}}{\partial \tilde{r}} \simeq \frac{2 k \lambda(z)}{\gamma r}
$$

\section{RMS coupled envelope equations}

Recall that the general expression for the coupled RMS equations is

$$
\begin{aligned}
& \sigma_{x}^{\prime \prime}-\frac{e}{p_{0} v_{0} \gamma_{0}^{2}} \frac{1}{\sigma_{x}}\left\langle x E_{x}\right\rangle-\frac{\tilde{\epsilon}_{x}^{2}}{\sigma_{x}^{3}}=0, \\
& \sigma_{y}^{\prime \prime}-\frac{e}{p_{0} v_{0} \gamma_{0}^{2}} \frac{1}{\sigma_{y}}\left\langle y E_{y}\right\rangle-\frac{\tilde{\epsilon}_{y}^{2}}{\sigma_{y}^{3}}=0,
\end{aligned}
$$

$$
\sigma_{z}^{\prime \prime}-\frac{e}{p_{0} v_{0} \gamma_{0}^{2}} \frac{1}{\sigma_{z}}\left\langle z E_{z}\right\rangle-\frac{\tilde{\epsilon}_{z}^{2}}{\sigma_{z}^{3}}=0,
$$

where $\tilde{\boldsymbol{\epsilon}}_{x}=\boldsymbol{\epsilon}_{x} / p_{0}, \tilde{\boldsymbol{\epsilon}}_{y}=\boldsymbol{\epsilon}_{y} / p_{0}$, and $\tilde{\boldsymbol{\epsilon}}_{z}=\boldsymbol{\epsilon}_{z} / \gamma_{0}^{2}$ are the RMS trace-space emittances defined as

$$
\begin{aligned}
& \tilde{\boldsymbol{\epsilon}}_{x}=\sqrt{\left\langle x^{2}\right\rangle\left\langle x^{\prime 2}\right\rangle-\left\langle x x^{\prime}\right\rangle^{2}}, \\
& \tilde{\boldsymbol{\epsilon}}_{y}=\sqrt{\left\langle y^{2}\right\rangle\left\langle y^{\prime 2}\right\rangle-\left\langle y y^{\prime}\right\rangle^{2}}, \\
& \tilde{\boldsymbol{\epsilon}}_{z}=\sqrt{\left\langle z^{2}\right\rangle\left\langle z^{\prime 2}\right\rangle-\left\langle z z^{\prime}\right\rangle^{2}} .
\end{aligned}
$$

The electric fields in the laboratory are not linear in $x, y$, and $z$. In the "long beam" limit, that is, for $a \ll \gamma a_{3}$, within the cylinder, the transverse electric field in the laboratory frame, is

$$
E_{r}=\gamma_{0} \tilde{E}_{r}=\frac{\lambda(z) r}{2 \pi \epsilon_{0} a^{2}}, \quad=\frac{3 e N r}{8 \pi \epsilon_{0} a^{2} a_{3}}\left(1-\frac{z^{2}}{a_{3}^{2}}\right) .
$$

The longitudinal field within the cylinder is

$$
\begin{aligned}
E_{z} & =\tilde{E}_{z}=-\frac{\lambda^{\prime}(z)}{2 \pi \epsilon_{0} \gamma^{2}}\left[\ln \left(\frac{2 \gamma \sqrt{a_{3}^{2}-z^{2}}}{a}\right)-\frac{1}{2}\left(1+\frac{r^{2}}{a^{2}}\right)\right] \\
& =\frac{3 e N z}{4 \pi \epsilon_{0} a_{3}^{3} \gamma^{2}}\left[\ln \left(\frac{2 \gamma \sqrt{a_{3}^{2}-z^{2}}}{a}\right)-\frac{1}{2}\left(1+\frac{r^{2}}{a^{2}}\right)\right] .
\end{aligned}
$$

Note that, since the fields are nonlinear functions of coordinates, the emittances will not be preserved. In the following we will ignore emittance growth is the envelope equation, that is the emittances are set equal to their initial values. For the transverse degree of freedom we have

$$
\left\langle x E_{x}\right\rangle=\left\langle y E_{y}\right\rangle=\frac{3 e N\left\langle x^{2}\right\rangle}{8 \pi \epsilon_{0} a^{2} a_{3}}\left(1-\frac{\left\langle z^{2}\right\rangle}{a_{3}^{2}}\right) .
$$

Using $\sigma_{z}^{2}=\left\langle z^{2}\right\rangle=a_{3}^{2} / 5$ and $\sigma_{x}^{2}=\left\langle x^{2}\right\rangle=\left\langle r^{2}\right\rangle / 2=a^{2} / 4$, where in general $\langle A\rangle$ is defined as

$$
\langle A\rangle=\frac{1}{\pi a^{2} e N} \int_{0}^{2 \pi} d \theta \int_{0}^{a} r d r \int_{-a_{3}}^{a_{3}} A(r, z) \lambda(z) d z,
$$

we get

$$
\left\langle x E_{x}\right\rangle=\frac{3 e N}{40 \pi \epsilon_{0} a_{3}} .
$$

For the longitudinal axis, we have

$$
\begin{aligned}
\left\langle z E_{z}\right\rangle= & -\frac{3 e N\left\langle z^{2}\right\rangle}{8 \pi \epsilon_{0} a_{3}^{3} \gamma^{2}}\left(1+\frac{\left\langle r^{2}\right\rangle}{a^{2}}\right)+\frac{3 e N}{4 \pi \epsilon_{0} a_{3}^{3} \gamma^{2}} \\
& \times\left\langle z^{2} \ln \left(\frac{2 \gamma \sqrt{a_{3}^{2}-z^{2}}}{a}\right)\right\rangle,
\end{aligned}
$$


where

$$
\left\langle z^{2} \ln \left(\frac{2 \gamma \sqrt{a_{3}^{2}-z^{2}}}{a}\right)\right\rangle=\frac{a_{3}^{2}}{5}\left[\ln \left(\frac{\gamma a_{3}}{a}\right)+2 \ln 2-1-\frac{1}{30}\right]
$$

and

$$
\left\langle z E_{z}\right\rangle=\frac{3 e N}{20 \pi \epsilon_{0} a_{3} \gamma^{2}} \ln \left(\frac{\gamma \alpha_{3} a_{3}}{a}\right)
$$

with $\alpha_{3} \simeq 0.6723$. Including $\left\langle x E_{x}\right\rangle,\left\langle y E_{y}\right\rangle$, and $\left\langle z E_{z}\right\rangle$ in the envelope equations, yields

$$
\begin{gathered}
\sigma_{x}^{\prime \prime}-\frac{3}{2} \frac{N r_{e}}{\gamma_{0}^{3} \beta_{0}^{2}} \frac{\lambda_{3}}{\sigma_{x} \sigma_{z}}-\frac{\tilde{\epsilon}_{x}^{2}}{\sigma_{x}^{3}}=0, \\
\sigma_{y}^{\prime \prime}-\frac{3}{2} \frac{N r_{e}}{\gamma_{0}^{3} \beta_{0}^{2}} \frac{\lambda_{3}}{\sigma_{y} \sigma_{z}}-\frac{\tilde{\epsilon}_{y}^{2}}{\sigma_{y}^{3}}=0, \\
\sigma_{z}^{\prime \prime}-\frac{3}{2} \frac{N r_{e}}{\gamma_{0}^{5} \beta_{0}^{2}} \frac{\lambda_{3}}{\sigma_{z}^{2}} g_{2}\left(\sigma_{x}, \gamma_{0} \sigma_{z}\right)-\frac{\tilde{\epsilon}_{z}^{2}}{\sigma_{z}^{3}}=0,
\end{gathered}
$$

where the classical electron radius $r_{e}=e^{2} /\left(4 \pi \epsilon_{0} m c^{2}\right)$ has been introduced together with

$$
g_{2}\left(\sigma_{x}, \gamma_{0} \sigma_{z}\right) \simeq 2 \ln \left(\frac{\gamma \alpha_{3}^{\prime} \sigma_{z}}{\sigma_{x}}\right)
$$

and the coefficients $\alpha_{3}^{\prime} \simeq 0.6$ and $\lambda_{3}=1 /(5 \sqrt{5})$.

[1] E. Esarey, P. Sprangle, J. Krall, and A. Ting, IEEE Trans. Plasma Sci. 24, 252 (1996).

[2] E. Esarey, R. F. Hubbard, W. P. Leemans, A. Ting, and P. Sprangle, Phys. Rev. Lett. 79, 2682 (1997).

[3] C. B. Schroeder, P. B. Lee, J. Wurtele, E. Esarey, and W. P. Leemans, Phys. Rev. E 59, 6037 (1999).

[4] G. Fubiani, E. Esarey, C. B. Schroeder, and W. P. Leemans, Phys. Rev. E 70, 016402 (2004).

[5] G. Fubiani, Ph.D. thesis, Lawrence Berkeley National Laboratory and University Paris XI, Orsay [LBNL Report No. 57514, 2005 (unpublished)].

[6] P. Lapostolle, A. Lombardi, E. Tanke, S. Valero, R. Garnett, and T. Wangler, Nucl. Instrum. Methods Phys. Res., Sect. A 379, 21 (1996).

[7] M. Reiser, Theory and Design of Charged Particle Beams (Wiley, New York, 1994).

[8] T. P. Wangler, RF Linear Accelerators (Wiley, New York, 1998), pp. 270-272.
[9] C. K. Birdsall and A.B. Langdon, Plasma Physics Via Computer Simulation (IOP Publishing, London, 1998).

[10] R. W. Hockney and J. W. Eastwood, Computer Simulation Using Particles (IOP Publishing, London, 1988).

[11] G. Fubiani, G. Dugan, W. P. Leemans, E. Esarey, and J. L. Bobin, in Advanced Accelerator Concepts, edited by C. E. Clayton and P. Muggli, AIP Conf. Proc. No. 647 (AIP, New York, 2002), pp. 203-212.

[12] S. Chandrasekhar, Ellipsoidal Figures of Equilibrium (Dover Publications, New York, 1969).

[13] G. Fubiani, E. Esarey, and W. P. Leemans, in Advanced Accelerator Concepts, edited by P. Colestock and S. Kelly, AIP Conf. Proc. No. 569 (AIP, New York, 2001), pp. 423435.

[14] A. W. Chao, R. Pitthan, T. Tajima, and D. Yeremian, Phys. Rev. ST Accel. Beams 6, 024201 (2003).

[15] B. van der Geer and M. de Loos, Ph.D. thesis, Technische Universiteit Eindhoven, 2001.

[16] F. Sacherer, IEEE Trans. Nucl. Sci. NS-18, 1105 (1971).

[17] R. Ryne, Technical Report LA-UR-95-391, Los Alamos National Laboratory, 1995.

[18] P. Lapostolle, IEEE Trans. Nucl. Sci. NS-18, 1101 (1971).

[19] J. Qiang, R. D. Ryne, S. Habib, and V. Decyk, J. Comput. Phys. 163, 434 (2000).

[20] R. C. Davidson and H. Qin, Physics of Intense Charged Particle Beams in High Energy Accelerators (World Scientific, Singapore, 2001).

[21] E. J. Konopinski, Electromagnetic Fields and Relativistic Particles (McGraw-Hill, New York, 1981).

[22] C. Gahn, G. D. Tsakiris, A. Pukhov, J. Meyer-ter-Vehn, G. Pretzler, P. Thirolf, D. Habs, and K. Witte, Phys. Rev. Lett. 83, 4772 (1999).

[23] W. P. Leemans, D. Rodgers, P.E. Catravas, C. G. R. Geddes, G. Fubiani, E. Esarey, B. A. Shadwick, R. Donahue, and A. Smith, Phys. Plasmas 8, 2510 (2001).

[24] W. P. Leemans, J. van Tilborg, J. Faure, C. G. R. Geddes, C. Tóth, C. B. Schroeder, E. Esarey, and G. Fubiani, Phys. Plasmas 11, 2899 (2004).

[25] V. Malka et al., Science 298, 1596 (2002).

[26] T. Hosokai, K. Kinoshita, A. Zhidkov, K. Nakamura, T. Watanabe, T. Ueda, H. Kotaki, M. Kando, K. Nakajima, and M. Uesaka, Phys. Rev. E 67, 036407 (2003).

[27] T. Hosokai, K. Kinoshita, A. Zhidkov, K. Nakamura, H. Kotaki, M. Kando, K. Nakajima, and M. Uesaka, Phys. Plasmas 11, L57 (2004).

[28] C. I. Moore, A. Ting, K. Krushelnick, E. Esarey, R. F. Hubbard, B. Hafizi, H. R. Burris, C. Manka, and P. Sprangle, Phys. Rev. Lett. 79, 3909 (1997).

[29] A. Ting, C. I. Moore, K. Krushelnick, C. Manka, E. Esarey, P. Sprangle, R. Hubbard, H. R. Burris, R. Fisher, and M. Baine, Phys. Plasmas 4, 1889 (1997).

[30] A. Modena et al., Nature (London) 377, 606 (1995).

[31] R. Wagner, S.-Y. Chen, A. Maksimchuk, and D. Umstadter, Phys. Rev. Lett. 78, 3125 (1997).

[32] X. Wang, M. Krishnan, N. Saleh, H. Wang, and D. Umstadter, Phys. Rev. Lett. 84, 5324 (2000).

[33] C. Geddes, C. Toth, J. van Tilborg, E. Esarey, C. Schroeder, D. Bruhwiler, C. Nieter, J. Cary, and W. Leemans, Nature (London) 431, 538 (2004).

[34] J. Faure, Y. Glinec, A. Pukhov, S. Kiselev, S. Gordienko, 
E. Lefebvre, J.-P. Rousseau, F. Burgy, and V. Malka, Nature (London) 431, 541 (2004).

[35] S. P. D. Mangles et al., Nature (London) 431, 535 (2004).

[36] C. Nieter and J. Cary, J. Comput. Phys. 196, 448 (2004).

[37] C. G. R. Geddes, Ph.D. thesis, University of California at Berkeley, 2005.

[38] F. S. Tsung (private communication).

[39] E. M. Lifshitz and L. D. Landau, The Classical Theory of Fields (Pergamon Press, New York, 1987).
[40] P. Clemmow and J. Dougherty, Electrodynamics of Particles and Plasmas (Addison-Wesley, Reading, MA, 1969).

[41] F. Rohrlich, Classical Charged Particles (AddisonWesley, Reading, MA, 1990).

[42] J. Huschilt, W. E. Baylis, D. Leiter, and G. Szamosi, Phys. Rev. D 7, 2844 (1973).

[43] J.D. Jackson, Classical Electrodynamics (Wiley, New York, 1975). 